Homology, Homotopy and Applications, vol.4(1), 2002, pp.103-162

\title{
EXTENSIONS OF SYMMETRIC CAT-GROUPS
}

\author{
D. BOURN AND E.M. VITALE \\ (communicated by Antonio Cegarra)
}

\begin{abstract}
This paper is an attempt to study extensions of symmetric categorical groups from a structural point of view. Using in a systematic way bilimits in the 2-category of symmetric categorical groups, we develop a theory which closely follows the classical theory of abelian group extensions. The basic results are established for any proper class of extensions, and a cohomological classification is obtained for those extensions whose epi part has a categorical section.
\end{abstract}

\section{Introduction}

Extensions of categorical groups have been extensively studied in $[\mathbf{2}, \mathbf{3}, \mathbf{4}, \mathbf{5}$, $6,7,8,9,10,14,20,25]$, and remarkable applications to the classification of homotopy types and to equivariant group cohomology have been found. In most of the works on this subject, the chosen class of "epimorphisms" between categorical groups is that of Grothendieck dense fibrations. This class does not have good 2categorical properties, basically because it is not stable under natural isomorphisms. We have therefore tried to replace the class of Grothendieck dense fibrations with a class having a better 2-categorical behaviour.

Since an epimorphism of abelian groups is a morphism with a set-theoretical section, our first idea was been to consider, as epimorphisms between symmetric categorical groups, those symmetric monoidal functors having a categorical section. This class is stable not only under natural isomorphisms, but also under bi-pullbacks. These facts allowed us to use in a systematic way bi-limits and bi-colimits, so that to develop the basic algebra for extensions in a way which closely follows the classical theory of abelian group extensions.

While doing this, we realized soon that, at least as far as the basic algebra is concerned, only few stability properties are needed. We decided then to rewrite the theory in terms of "proper classes" of extensions, having as examples extensions with a categorical section, extensions with a graph-theoretical section, and also the more general class of extensions in which the epi part is simply a functor essentially surjective on objects. This last class seems to be the most general one which supports the constructions of basic algebra. It has been independently considered by A. Rousseau in his Ph. D. Thesis [20], where extensions of (not necessarily symmetric)

Received April 5, 2002, revised October 24, 2002; published on November 20, 2002.

2000 Mathematics Subject Classification: 18D10, 18G99, $20 \mathrm{~L} 05$.

Key words and phrases: symmetric categorical groups, extensions, bilimits, homological algebra. (c) 2002, D. Bourn and E.M. Vitale. Permission to copy for private use granted. 
categorical groups are classified following Breen-Grothendieck approach in terms of monoidal fibrations of bitorsors.

The present paper is organized as follows:

After recalling basic facts on categorical groups (Section 1), in Section 2 we give the definition of extension of symmetric categorical groups and we show that the 2category $\operatorname{EXT}(\mathbb{C}, \mathbb{A})$ of extensions of $\mathbb{A}$ by $\mathbb{C}$ is a 2 -groupoid. In Section 3 we compare extensions of symmetric categorical groups with extensions of abelian groups. Section 4 is devoted to trivial and split extensions. In Section 5 we discuss bi-pullbacks and bi-pushouts of symmetric categorical groups. They are used in Sections 6 and 7 to compare EXT and Hom and to show that EXT measures whether a morphism can be extended or lifted. Sections 8 and 9 contain definition, basic facts and some examples of proper classes of extensions. In Section 10, we establish the fundamental 2-exact Hom-Ext sequences obtained by an extension. Section 11 is devoted to projective (and injective) objects. In Section 12 we show that EXT measures the non-exactness of Hom. In Section 13 we define the 2-dimensional analogue of Baer sum, making $\operatorname{Ext}(\mathbb{C}, \mathbb{A})$ into a symmetric categorical group. The last two sections are devoted to a cohomological classifications of $\mathcal{F}$-extensions (i.e. those extensions of symmetric categorical groups whose epi part has a categorical section). We define a convenient notion of cobord and symmetric cocycle and we obtain a categorical equivalence between the symmetric categorical group of $\mathcal{F}$-extensions and the symmetric categorical group of cocycles modulo cobords.

All along the paper, we restrict our attention to symmetric categorical groups and monoidal functors compatible with the symmetry. Several results contain a part stated in terms of bi-limits only (or proved using only bi-limits) and a dual part involving bi-colimits. It is a general fact that the part involving only bi-limits holds also for not necessarily symmetric categorical groups and arbitrary monoidal functors.

To end this introduction, let us point out two major open problems.

Clearly, working with symmetric categorical groups instead of braided categorical groups, we loss some relevant examples. The reason why we restrict our attention to the symmetric case is that the cokernel of a morphism (as well as basic properties on 2 -exact sequences based on the duality kernel-cokernel, see [16]) is a main ingredient in our analysis, and its description is known, up to now, only for morphisms between symmetric categorical groups. Moreover, several definitions and constructions we use seem much more delicate if we have only a braiding instead of a symmetry (they are so delicate that we suspect that the right context to study the non-symmetric theory could be that of bigroupoids, instead of categorical groups).

Another problem concerns projective objects (in the sense of Definition 11.1) in the 2-category of symmetric categorical groups. The notion of projectivity is crucial in the classical theory, but, unfortunately, we do not know if the 2-category of symmetric categorical groups has enough projective objects. (It would be interesting to solve this problem in order to appreciate the strong specialization done in Sections 14 and 15 , where we consider only $\mathcal{F}$-extensions.)

Finally, we would like to thank the members of the Granada school in category theory for several stimulating and useful discussions the second author had with 
them when he was visiting Granada in summer 2001. We also thank the referee for his help in comparing $\mathcal{F}$-extensions with the classification of extensions established in $[\mathbf{2 0}]$.

\section{The 2-category SCG}

Let us fix some notations. In any category, the composition is written diagrammatically, that is $X \stackrel{f}{\longrightarrow} Y \stackrel{g}{\longrightarrow} Z$ is written $f \cdot g$. The identity arrow is $1=1_{X}: X \rightarrow X$ (but the identity natural transformation on a functor $F$ is called $F$ ). If $\mathbb{C}$ is a monoidal category, we write $I=I_{\mathbb{C}}$ for the unit object, $\otimes$ for the tensor product, $a=a_{X, Y, Z}:(X \otimes Y) \otimes Z \rightarrow X \otimes(Y \otimes Z)$ for the associativity isomorphism, $l=l_{X}: I \otimes X \rightarrow X$ and $r=r_{Y}: Y \otimes I \rightarrow Y$ for the unit isomorphisms. (Ab)using the coherence theorem for monoidal categories, we often assume the associativity isomorphism to be the identity. If $\mathbb{C}$ is symmetric, we write $\gamma=\gamma_{X, Y}: X \otimes Y \rightarrow Y \otimes X$ for the symmetry. If $F: \mathbb{C} \rightarrow \mathbb{D}$ is a monoidal functor, we denote its monoidal structure by $F_{X, Y}: F(X) \otimes F(Y) \rightarrow F(X \otimes Y)$ and $F_{I}: I \rightarrow F(I)$. For any category $\mathbb{C}$, we write $\pi_{0} \mathbb{C}$ for the (possibly large) set of isomorphism classes of objects. If $\mathbb{C}$ is monoidal, $\pi_{1} \mathbb{C}$ is the commutative (see $[\mathbf{1 8}]$ ) monoid $\mathbb{C}(I, I)$. A categorical group (cat-group, for short) is a monoidal groupoid in which each object is regular, i.e. it is invertible, up to isomorphism, with respect to the tensor product. If $\mathbb{G}$ is a cat-group, we fix, for each object $X$, a dual object $X^{*}$, with unit and counit $\eta_{X}: I \rightarrow X \otimes X^{*}, \epsilon_{X}: X^{*} \otimes X \rightarrow I$. If $f: X \rightarrow Y$ is in $\mathbb{C}$, $f^{*}: Y^{*} \rightarrow X^{*}$ is given by

$$
\begin{aligned}
Y^{*} \simeq Y^{*} \otimes I & \stackrel{1 \otimes \eta_{X}}{\longrightarrow} Y^{*} \otimes\left(X \otimes X^{*}\right) \stackrel{1 \otimes(f \otimes 1)}{\longrightarrow} Y^{*} \otimes\left(Y \otimes X^{*}\right) \simeq \\
& \simeq\left(Y^{*} \otimes Y\right) \otimes X^{*} \stackrel{\epsilon_{Y} \otimes 1}{\longrightarrow} I \otimes X^{*} \simeq X^{*} .
\end{aligned}
$$

Basic facts on monoidal categories and cat-groups can be found in $[\mathbf{1 1}, \mathbf{1 2}, \mathbf{1 3}, \mathbf{1 5}$, $16,19,21,23,24,26]$.

The 2-category SCG has symmetric cat-groups as objects, monoidal functors compatible with the symmetry as 1-cells, and monoidal natural transformations as 2cells (observe that they are natural isomorphisms, because cat-groups are groupoids). Note that $\pi_{0}$ and $\pi_{1}$ extend to two 2-functors from SCG to the discrete 2-category $\mathrm{Ab}$ of abelian groups. Moreover, a 1-cell $F: \mathbb{A} \rightarrow \mathbb{B}$ in SCG is an equivalence if and only if $\pi_{0}(F)$ and $\pi_{1}(F)$ are isomorphisms in $\mathrm{Ab}$ (see [16]).

Let us recall now, from [16], the universal property and a construction for kernels and cokernels in SCG. (As a matter of convention, each time that we consider some kind of limit or colimit in SCG, it is to be understood in the sense of bi-limit or bi-colimit, see $[\mathbf{2 2}]$.) Given a 1-cell $\Sigma: \mathbb{B} \rightarrow \mathbb{C}$, its kernel is given by a triple $\left(\operatorname{Ker} \Sigma, e_{\Sigma}, \epsilon_{\Sigma}\right)$

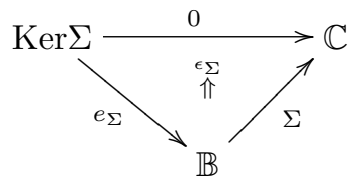


(where $0: \operatorname{Ker} \Sigma \rightarrow \mathbb{C}$ is defined by $0(f)=1_{I}$ for each arrow $f$ in $\operatorname{Ker} \Sigma$ ) such that, for any other triple $(\mathbb{D}, F, \varphi)$

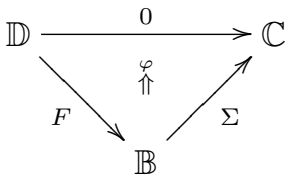

there are $F^{\prime}: \mathbb{D} \rightarrow \operatorname{Ker} \Sigma$ and $\varphi^{\prime}: F^{\prime} \cdot e_{\Sigma} \Rightarrow F$ such that the following diagram commutes

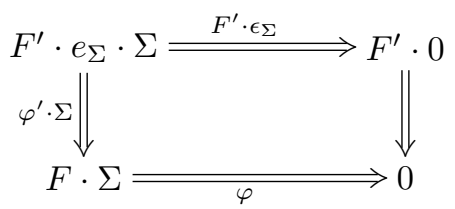

Moreover, if $F^{\prime \prime}: \mathbb{D} \rightarrow \operatorname{Ker} \Sigma$ and $\varphi^{\prime \prime}: F^{\prime} \cdot e_{\Sigma} \Rightarrow F$ make commutative the analogous diagram, then there is a unique $\psi: F^{\prime} \Rightarrow F^{\prime \prime}$ making commutative the following diagram

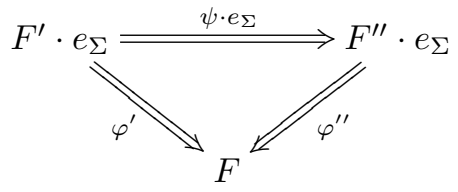

As any bilimit, the kernel is determined, up to equivalence, by its universal property. It can be described as the comma category having as objects pairs of the form $\left(X \in \mathbb{B}, \epsilon_{X}: \Sigma(X) \rightarrow I\right)$. The functor $e_{\Sigma}$ forgets $\epsilon_{X}$ and $\epsilon_{\Sigma}\left(X, \epsilon_{X}\right)$ is $\epsilon_{X}$. In fact, this description could be called the standard kernel, because it satisfies another universal property: if $F$ and $\varphi$ are as before, there is a unique $F^{\prime}$ such that $F^{\prime} \cdot e_{\Sigma}=F$ and $F^{\prime} \cdot \epsilon_{\Sigma}=\varphi$. We always use the first universal property, but the second one is sometimes useful to avoid $\varphi^{\prime}$ and then to simplify notations.

The cokernel is defined by the dual universal property. In the following diagram, we fix the notations for the cokernel of a 1-cell $\Gamma: \mathbb{A} \rightarrow \mathbb{B}$

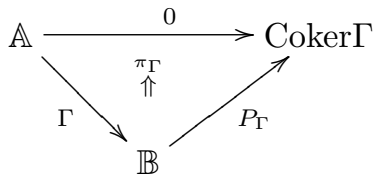

Objects of Coker $\Gamma$ are those of $\mathbb{B}$. An arrow $X \rightarrow Y$ in Coker $\Gamma$ is an equivalence class of pairs of the form $(f, A)$, with $A$ an object of $\mathbb{A}$ and $f: X \rightarrow Y \otimes \Gamma(A)$ an arrow in $\mathbb{B}$. Two pairs $(f, A)$ and $\left(f^{\prime}, A^{\prime}\right)$ are equivalent if there is $g: A \rightarrow A^{\prime}$ in $\mathbb{A}$ such that $f \cdot\left(1_{Y} \otimes \Gamma g\right)=f^{\prime}$. Once again, we get in this way a standard cokernel. 


\section{The 2-category of extensions}

We want now to define extensions of symmetric cat-groups. For this, consider the following diagram in SCG :

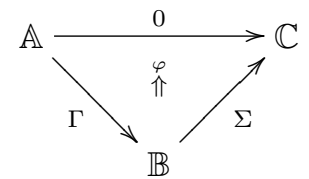

and the corresponding factorizations through the kernel of $\Sigma$ and the cokernel of $\Gamma$

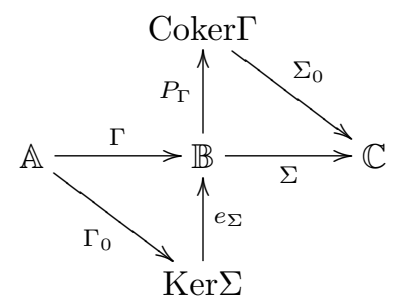

Recall, from [16], that the triple $(\Gamma, \varphi, \Sigma)$ is 2 -exact when $\Gamma_{0}$ is full and essentially surjective on objects or, equivalently, when $\Sigma_{0}$ is full and faithful.

Proposition 2.1. The following conditions are equivalent :

1) The triple $(\Gamma, \varphi, \Sigma)$ is 2-exact, $\Gamma$ is faithful and $\Sigma$ is essentially surjective;

2) $\Gamma_{0}$ is an equivalence and $\Sigma$ is essentially surjective;

3) $\Gamma$ is faithful and $\Sigma_{0}$ is an equivalence.

Proof. Obvious, because $P_{\Gamma}$ is essentially surjective and $e_{\Sigma}$ is faithful.

We are ready to give the definition of extension (compare with Definition 3.2.1 in $[20])$.

Definition 2.2. Let $\mathbb{A}, \mathbb{C}$ be in $\mathrm{SCG}$;

1) An extension of $\mathbb{A}$ by $\mathbb{C}$ is a diagram $(\Gamma, \varphi, \Sigma)$ in $\mathrm{SCG}$

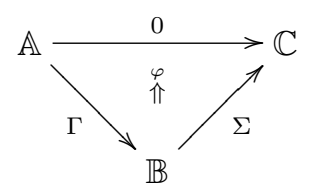

which satisfies the equivalent conditions of Proposition 2.1. We write also $(\Gamma, \varphi, \Sigma): \mathbb{A} \rightarrow \mathbb{B} \rightarrow \mathbb{C}$ for such an extension;

2) If $(\Gamma, \varphi, \Sigma)$ and $\left(\Gamma^{\prime}, \varphi^{\prime}, \Sigma^{\prime}\right)$ are two extensions of $\mathbb{A}$ by $\mathbb{C}$, a 1-cell

$$
(\alpha, \beta, \gamma):(\Gamma, \varphi, \Sigma) \rightarrow\left(\Gamma^{\prime}, \varphi^{\prime}, \Sigma^{\prime}\right)
$$


is given by a 1-cell $\beta$ and two 2-cells $\alpha, \gamma$ in $\mathrm{SCG}$ as in the following diagram

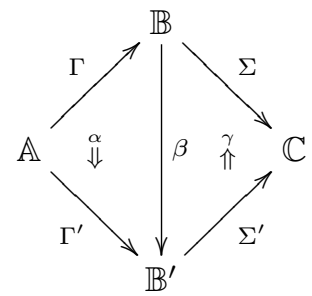

such that the following diagram commutes

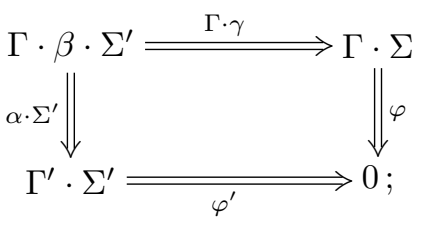

3) If $(\alpha, \beta, \gamma),\left(\alpha^{\prime}, \beta^{\prime}, \gamma^{\prime}\right):(\Gamma, \varphi, \Sigma) \rightarrow\left(\Gamma^{\prime}, \varphi^{\prime}, \Sigma^{\prime}\right)$ are 1-cells, a 2-cell

$$
b:(\alpha, \beta, \gamma) \Rightarrow\left(\alpha^{\prime}, \beta^{\prime}, \gamma^{\prime}\right)
$$

is a 2-cell $b: \beta \Rightarrow \beta^{\prime}$ in SCG such that the following diagrams commute
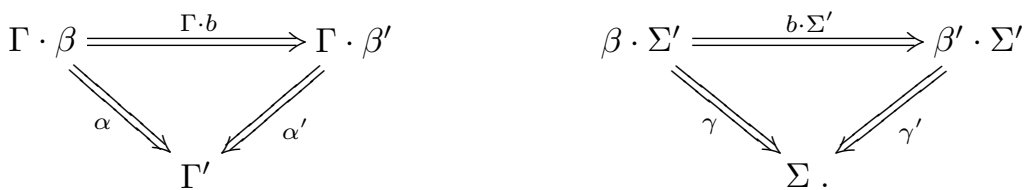

Proposition 2.3. With the obvious compositions and identities, the data of Definition 2.2 define a 2-category $\operatorname{EXT}(\mathbb{C}, \mathbb{A})$.

We write $\operatorname{Ext}(\mathbb{C}, \mathbb{A})$ for the classifying category of $\operatorname{EXT}(\mathbb{C}, \mathbb{A})$, i.e. $\operatorname{Ext}(\mathbb{C}, \mathbb{A})$ has the same objects as $\operatorname{EXT}(\mathbb{C}, \mathbb{A})$ and, as arrows, 2-isomorphism classes of 1-cells in $\operatorname{EXT}(\mathbb{C}, \mathbb{A})$.

Example 2.4. Let $F: \mathbb{A} \rightarrow \mathbb{B}$ be a morphism in SCG. The following diagrams are extensions
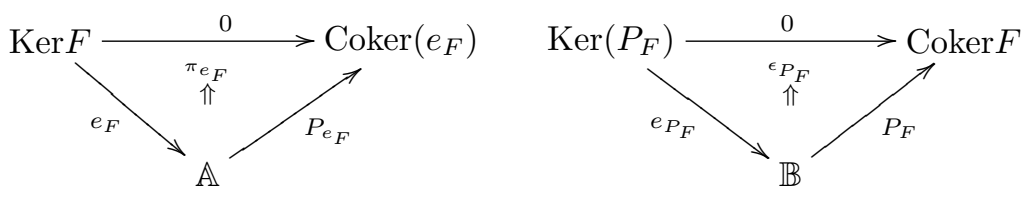

Our first result on extensions will be the 2-dimensional analogue of the Short Five Lemma, that is the fact that $\operatorname{EXT}(\mathbb{C}, \mathbb{A})$ is a 2-groupoid. This has been independently proved also in $[\mathbf{2 0}]$. We sketch the proof for the reader's convenience and because we deduce this fact from a slightly more general argument. We need a notation: if $A$ is an abelian group, we write $A[1]$ for the symmetric cat-group with 
only one object and such that $\pi_{1}(A[1])=A$; we write $A[0]$ for the discrete symmetric cat-group such that $\pi_{0}(A[0])=A$. Both $(-)[0]$ and $(-)[1]$ extend to morphisms. (Of course, $A[0]$ and $A[1]$ are strict cat-groups. Compare with [1], where classical homological algebra is developed in terms of higher strict $n$-cat-groups.)

Lemma 2.5. Let $(f, g): A \rightarrow B \rightarrow C$ be an exact sequence of abelian groups.

1) If $f$ is a monomorphism, then $(f[0],=, g[0]): A[0] \rightarrow B[0] \rightarrow C[0]$ is a 2-exact sequences of symmetric cat-groups;

2) If $g$ is an epimorphism, than $(f[1],=, g[1]): A[1] \rightarrow B[1] \rightarrow C[1]$ is a 2-exact sequences of symmetric cat-groups.

Proposition 2.6. Consider a morphism in SCG together with its kernel and its cokernel,

$$
\operatorname{Ker} F \stackrel{e_{F}}{\longrightarrow} \mathbb{G} \stackrel{F}{\longrightarrow} \mathbb{H} \stackrel{P_{F}}{\longrightarrow} \operatorname{Coker} F \text {. }
$$

Then the following diagram (where $\mathbf{1}$ is the symmetric cat-group with only one arrow, and $\lambda$ and $\mu$ are defined in the proof) is a 2-exact sequence of symmetric cat-groups

$$
\begin{gathered}
\mathbf{1} \longrightarrow \pi_{1}(\operatorname{Ker} F)[0] \stackrel{\pi_{1}\left(e_{F}\right)[0]}{\longrightarrow} \pi_{1}(\mathbb{G})[0] \stackrel{\pi_{1}(F)[0]}{\longrightarrow} \\
\pi_{1}(\mathbb{H})[0] \stackrel{\lambda}{\longrightarrow} \operatorname{Ker} F \stackrel{e_{F}}{\longrightarrow} \stackrel{F}{\longrightarrow} \operatorname{Coker} F \\
\stackrel{P_{F}}{\longrightarrow} \pi_{0}(\mathbb{G})[1] \stackrel{\pi_{0}(F)[1]}{\longrightarrow} \pi_{0}(\mathbb{H})[1] \stackrel{\pi_{0}\left(P_{F}\right)[1]}{\longrightarrow} \pi_{0}(\operatorname{Coker} F)[1] \stackrel{\text { 1 }}{\longrightarrow}
\end{gathered}
$$

Proof. Since $\pi_{1}$ preserves kernels, $\pi_{0}$ preserves cokernels and both send 2-exact sequences into exact sequences $[\mathbf{2 7}], 2$-exactness in $\pi_{1}(\operatorname{Ker} F)[0], \pi_{1}(\mathbb{G})[0], \pi_{0}(\mathbb{H})[1]$ and $\pi_{0}(\operatorname{Coker} F)[1]$ follows from the previous lemma. 2-exactness in $\mathbb{G}$ and $\mathbb{H}$ is obvious. It remains to check 2-exactness in $\pi_{1}(\mathbb{H})[0], \operatorname{Ker} F, \operatorname{Coker} F$ and $\pi_{0}(\mathbb{G})[1]$. This is straightforward, once $\lambda$ and $\mu$ defined. From [16], recall that $\pi_{0}($ Ker $)$ and $\pi_{1}($ Coker $F)$ are isomorphic (in fact, they are equal if we use the description of Ker and Coker given in Section 1).

The functor $\lambda$ corresponds to $\pi_{1}\left(P_{F}\right): \pi_{1}(\mathbb{H}) \rightarrow \pi_{1}(\operatorname{Coker} F)=\pi_{0}(\operatorname{Ker} F)$; explicitly, $\lambda: \pi_{1}(\mathbb{H})[0] \rightarrow \operatorname{Ker} F$ sends $h: I \rightarrow I$ to $\left(I, F_{I}^{-1} \cdot h: F(I) \rightarrow I\right)$.

The functor $\mu$ corresponds to $\pi_{0}\left(e_{F}\right): \pi_{1}(\operatorname{Coker} F)=\pi_{0}(\operatorname{Ker} F) \rightarrow \pi_{0}(\mathbb{G})$; explicitly, $\mu: \operatorname{Coker} F \rightarrow \pi_{0}(\mathbb{G})[1]$ sends $[f, G]: H_{1} \rightarrow H_{2}$ to $[G]: * \rightarrow *$.

Corollary 2.7. Let $F: \mathbb{G} \rightarrow \mathbb{H}$ be a morphism in $S C G$. The following is an exact sequence of abelian groups

$$
\begin{aligned}
& 0 \longrightarrow \pi_{1}(\operatorname{Ker} F) \stackrel{\pi_{1}\left(e_{F}\right)}{\longrightarrow} \pi_{1}(\mathbb{G}) \stackrel{\pi_{1}(F)}{\longrightarrow} \pi_{1}(\mathbb{H}) \stackrel{\pi_{1}\left(P_{F}\right)}{\longrightarrow} \pi_{1}(\operatorname{Coker} F) \\
& 0< \\
& 0
\end{aligned}
$$


Proposition 2.8. Let $\mathbb{A}$ and $\mathbb{C}$ be in $\mathrm{SCG}$.

1) The 2-category $\operatorname{EXT}(\mathbb{C}, \mathbb{A})$ is a 2-groupoid, i.e. each 2-cell is an isomorphism and each 1-cell is an equivalence;

2) The category $\operatorname{Ext}(\mathbb{C}, \mathbb{A})$ is a groupoid.

Proof. 1) We use the notations of Definition 2.2. To prove that 2-cells are isomorphisms, one simply checks that, if $b:(\alpha, \beta, \gamma) \Rightarrow\left(\alpha^{\prime}, \beta^{\prime}, \gamma^{\prime}\right)$ is a 2-cell in $\operatorname{EXT}(\mathbb{C}, \mathbb{A})$, then $b^{-1}: \beta^{\prime} \Rightarrow \beta$ is a 2 -cell $\left(\alpha^{\prime}, \beta^{\prime}, \gamma^{\prime}\right) \Rightarrow(\alpha, \beta, \gamma)$ in $\operatorname{EXT}(\mathbb{C}, \mathbb{A})$. Consider now a 1cell $(\alpha, \beta, \gamma):(\Gamma, \varphi, \Sigma) \rightarrow\left(\Gamma^{\prime}, \varphi^{\prime}, \Sigma^{\prime}\right)$ in $\operatorname{EXT}(\mathbb{C}, \mathbb{A})$. Consider also the factorizations $\bar{\beta}: \operatorname{Ker} \Sigma \rightarrow \operatorname{Ker} \Sigma^{\prime}$ and $\beta$ : Coker $\Gamma \rightarrow \operatorname{Coker} \Gamma^{\prime}$ induced, respectively, by $(\beta, \gamma)$ and $(\alpha, \beta)$. A straightforward direct computation shows that the following four squares commute

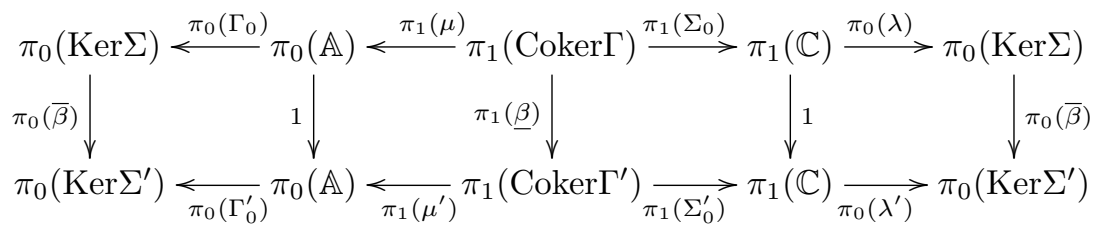

The commutativity of the first and the third square means that $\pi_{0}(\bar{\beta})$ and $\pi_{1}(\underline{\beta})$ are isomorphisms. Now we can particularize Corollary 2.7 taking, as $F$, the functors $\Sigma$ and $\Sigma^{\prime}$. We obtain the following commutative diagram with exact rows

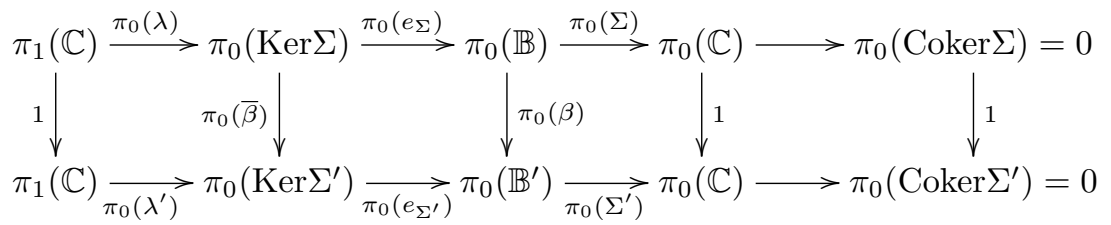

(the zeros are due to the fact that $\Sigma$ and $\Sigma^{\prime}$ are essentially surjective). By the Five Lemma, $\pi_{0}(\beta)$ is an isomorphism. In an analogous way, we can particularize Corollary 2.7 taking, as $F$, the functors $\Gamma$ and $\Gamma^{\prime}$. We have the following commutative diagram with exact rows

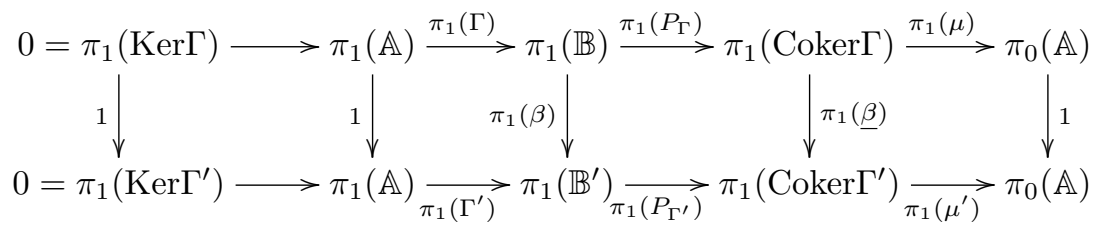

(where the zeros are due to the fact that $\Gamma$ and $\Gamma^{\prime}$ are faithful). The Five Lemma implies now that $\pi_{1}(\beta)$ is an isomorphism. This implies that $\beta: \mathbb{B} \rightarrow \mathbb{B}^{\prime}$ is an equivalence in SCG. Consider the adjoint equivalence in SCG

$$
\beta^{-1}: \mathbb{B}^{\prime} \rightarrow \mathbb{B} ; \eta: 1_{\mathbb{B}} \Rightarrow \beta \cdot \beta^{-1} ; \epsilon: \beta^{-1} \cdot \beta \Rightarrow 1_{\mathbb{B}^{\prime}}
$$


and put

$$
\begin{gathered}
x^{-1}: \Gamma \stackrel{\Gamma \cdot \eta}{\Longrightarrow} \Gamma \cdot \beta \cdot \beta^{-1} \stackrel{\alpha \cdot \beta^{-1}}{\Longrightarrow} \Gamma^{\prime} \cdot \beta^{-1} \\
y^{-1}: \Sigma^{\prime} \stackrel{\epsilon^{-1} \cdot \Sigma^{\prime}}{\Longrightarrow} \beta^{-1} \cdot \beta \cdot \Sigma^{\prime} \stackrel{\beta^{-1} \cdot \gamma}{\Longrightarrow} \beta^{-1} \cdot \Sigma
\end{gathered}
$$

It is now straightforward, using triangular identities for $(\eta, \epsilon)$, to check that $\left(x, \beta^{-1}, y\right):\left(\Gamma^{\prime}, \varphi^{\prime}, \Sigma^{\prime}\right) \rightarrow(\Gamma, \varphi, \Sigma)$ is a 1 -cell and $\eta, \epsilon$ are 2 -cells in $\operatorname{EXT}(\mathbb{C}, \mathbb{A})$.

2) Obvious from 1).

\section{Abelian group extensions}

The comparison between abelian group extensions and extensions of symmetric cat-groups is the object of this short section. First, observe that we can complete Lemma 2.5 in the following way.

Lemma 3.1. Let $(f, g): A \rightarrow B \rightarrow C$ be an extension of abelian groups. Then $(f[0],=, g[0]): A[0] \rightarrow B[0] \rightarrow C[0]$ and $(f[1],=, g[1]): A[1] \rightarrow B[1] \rightarrow C[1]$ are extensions of symmetric cat-groups.

Proof. If $0 \rightarrow A \rightarrow B \rightarrow C \rightarrow 0$ is exact, then $\mathbf{1} \rightarrow A[0] \rightarrow B[0] \rightarrow C[0] \rightarrow \mathbf{1}$ is 2-exact. In particular, the 2-exactness in $A[0]$ means that $f[0]$ is full and faithful, and the 2-exactness in $C[0]$ means that $g[0]$ is full and essentially surjective. The same argument works for $A[1] \rightarrow B[1] \rightarrow C[1]$.

The converse is not true, in the sense that if $(\Gamma, \varphi, \Sigma): \mathbb{A} \rightarrow \mathbb{B} \rightarrow \mathbb{C}$ is an extension of symmetric cat-groups, then in general neither $\left(\pi_{0}(\Gamma), \pi_{0}(\Sigma)\right): \pi_{0}(\mathbb{A}) \rightarrow \pi_{0}(\mathbb{B}) \rightarrow$ $\pi_{0}(\mathbb{C})$ nor $\left(\pi_{1}(\Gamma), \pi_{1}(\Sigma)\right): \pi_{1}(\mathbb{A}) \rightarrow \pi_{1}(\mathbb{B}) \rightarrow \pi_{1}(\mathbb{C})$ are extensions of abelian groups (respectively because $\pi_{0}(\Gamma)$ in general is not injective and $\pi_{1}(\Sigma)$ in general is not surjective.

Lemma 3.2. Let $(\Gamma, \varphi, \Sigma): \mathbb{A} \rightarrow \mathbb{B} \rightarrow \mathbb{C}$ be an extension of symmetric cat-groups.

1) If $\pi_{1}(\mathbb{A})=0=\pi_{1}(\mathbb{C})$ then $\pi_{1}(\mathbb{B})=0$ and $\left(\pi_{0}(\Gamma), \pi_{0}(\Sigma)\right): \pi_{0}(\mathbb{A}) \rightarrow \pi_{0}(\mathbb{B}) \rightarrow$ $\pi_{0}(\mathbb{C})$ is an extension of abelian groups;

2) If $\pi_{0}(\mathbb{A})=0=\pi_{0}(\mathbb{C})$ then $\pi_{0}(\mathbb{B})=0$ and $\left(\pi_{1}(\Gamma), \pi_{1}(\Sigma)\right): \pi_{1}(\mathbb{A}) \rightarrow \pi_{1}(\mathbb{B}) \rightarrow$ $\pi_{1}(\mathbb{C})$ is an extension of abelian groups.

Proof. 1) If we apply $\pi_{1}$, we get an exact sequence $0 \rightarrow \pi_{1}(\mathbb{B}) \rightarrow 0$, so that $\pi_{1}(\mathbb{B})=$ 0 . Moreover, since $\pi_{1}(\mathbb{C})=0$, then $e_{\Sigma}$ is full (see $[\mathbf{1 6}]$ ). But then also $\Gamma$ is full, so that $\pi_{0}(\Gamma)$ is injective.

2) If we apply $\pi_{0}$, we get an exact sequence $0 \rightarrow \pi_{0}(\mathbb{B}) \rightarrow 0$, so that $\pi_{0}(\mathbb{B})=0$. Moreover, since $\pi_{0}(\mathbb{A})=0$, then $P_{\Gamma}$ is full (see [16]). But then also $\Sigma$ is full, so that $\pi_{1}(\Sigma)$ is surjective.

Note that, in the situation of Lemma 3.2, if $\pi_{1}(\mathbb{B})=0$, then $\pi_{1}(\mathbb{A})=0$ (because $\pi_{1}$ preserves kernels), but in general $\pi_{1}(\mathbb{C}) \neq 0$ (because the cokernel of a morphism 
between discrete cat-groups in general is not dicrete). Dually, if $\pi_{0}(\mathbb{B})=0$, then $\pi_{0}(\mathbb{C})=0$ (because $\pi_{0}$ preserves cokernels), but in general $\pi_{0}(\mathbb{A}) \neq 0$.

We can summarize the previous discussion in the following proposition.

Proposition 3.3. Let $A$ and $C$ be two abelian groups. The (discrete) 2-groupoids $\operatorname{EXT}(C[0], A[0]), \operatorname{EXT}(C[1], A[1])$ and $\operatorname{EXT}(C, A)$ (the classical groupoid of extensions of $A$ by $C$ ) are equivalent.

\section{Trivial extensions}

Consider two symmetric cat-groups $\mathbb{A}$ and $\mathbb{C}$; the product category $\mathbb{A} \times \mathbb{C}$ with the obvious projections and injections

$$
\mathbb{A} \stackrel{p_{\mathbb{A}}}{\longleftarrow} \mathbb{A} \times \mathbb{C} \stackrel{p_{\mathbb{C}}}{\longrightarrow} \mathbb{C} \stackrel{i_{\mathbb{A}}}{\longrightarrow} \mathbb{A} \times \mathbb{C} \stackrel{i_{\mathbb{C}}}{\longleftarrow} \mathbb{C}
$$

satisfies the universal properties of the product and of the coproduct in SCG.

Example 4.1. If $\mathbb{A}, \mathbb{C}$ are in $\mathrm{SCG}$, then

$$
T(\mathbb{A}, \mathbb{C}): \mathbb{A} \stackrel{i_{\mathbb{A}}}{\longrightarrow} \mathbb{A} \times \mathbb{C} \stackrel{p_{\mathbb{C}}}{\longrightarrow} \mathbb{C}
$$

(with the identity 2 -cell $i_{\mathbb{A}} \cdot p_{\mathbb{C}}=0$ ) is an extension of $\mathbb{A}$ by $\mathbb{C}$. We call it the trivial extension of $\mathbb{A}$ by $\mathbb{C}$.

Definition 4.2. An extension $(\Gamma, \varphi, \Sigma): \mathbb{A} \rightarrow \mathbb{B} \rightarrow \mathbb{C}$ is a split extension of $\mathbb{A}$ by $\mathbb{C}$ (or, in short, splits) if it is equivalent, in $\operatorname{EXT}(\mathbb{C}, \mathbb{A})$, to the trivial extension $T(\mathbb{A}, \mathbb{C})$.

\section{Lemma 4.3 .}

1) Let

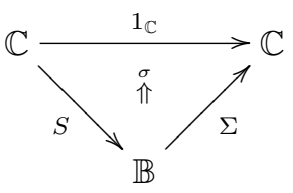

be in $\mathrm{SCG}$; then $\left(e_{\Sigma}, \epsilon_{\Sigma}, \Sigma\right): \operatorname{Ker} \Sigma \rightarrow \mathbb{B} \rightarrow \mathbb{C}$ is a split extension of $\operatorname{Ker} \Sigma$ by $\mathbb{C}$;

2) Let

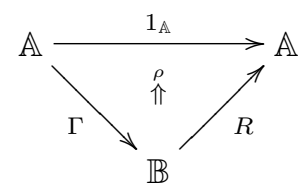

be in $\mathrm{SCG}$; then $\left(\Gamma, \pi_{\Gamma}, P_{\Gamma}\right): \mathbb{A} \rightarrow \mathbb{B} \rightarrow$ Coker $\Gamma$ is a split extension of $\mathbb{A}$ by CokerГ. 
Proof. We give a detailed proof of part 1) (part 2) is similar) because it is our first example of proof using the universal property of a (bi)limit. We need a 1-cell in $\operatorname{EXT}(\mathbb{C}, \operatorname{Ker} \Sigma)$

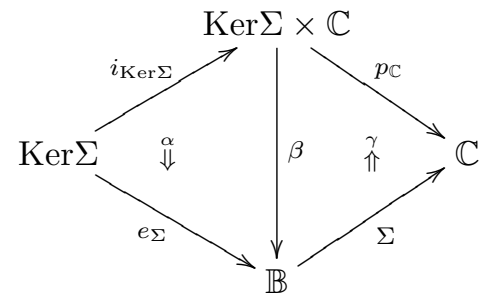

By the universal property of the coproduct $\operatorname{Ker} \Sigma \times \mathbb{C}$, we get a 1-cell $\beta$ and two 2-cells $\alpha, \delta$ in SCG

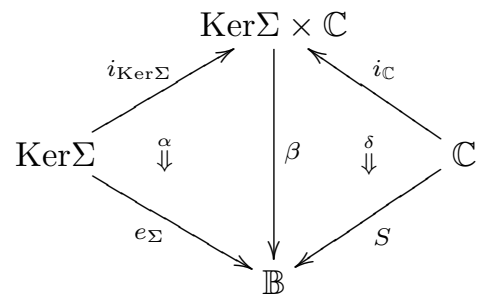

Now we have two 2-cells in SCG

$$
\begin{gathered}
\gamma_{1}: i_{\mathrm{Ker} \Sigma} \cdot \beta \cdot \Sigma \stackrel{\alpha \cdot \Sigma}{\Longrightarrow} e_{\Sigma} \cdot \Sigma \stackrel{\epsilon_{\Sigma}}{\Longrightarrow} 0 \Longrightarrow i_{\mathrm{Ker} \Sigma} \cdot p_{\mathbb{C}} \\
\gamma_{2}: i_{\mathbb{C}} \cdot \beta \cdot \Sigma \Longrightarrow \delta \cdot \Sigma \Longrightarrow i_{\mathbb{C}} \cdot p_{\mathbb{C}}
\end{gathered}
$$

By the universal property of the coproduct $\operatorname{Ker} \Sigma \times \mathbb{C}$, there exists a unique 2-cell $\gamma: \beta \cdot \Sigma \Rightarrow p_{\mathbb{C}}$ in SCG such that $i_{\operatorname{Ker} \Sigma} \cdot \gamma=\gamma_{1}$ and $i_{\mathbb{C}} \cdot \gamma=\gamma_{2}$. It remains to check that $(\alpha, \beta, \gamma): T(\operatorname{Ker} \Sigma, \mathbb{C}) \rightarrow\left(e_{\Sigma}, \epsilon_{\Sigma}, \Sigma\right)$ is a 1 -cell in $\operatorname{EXT}(\mathbb{C}, \operatorname{Ker} \Sigma)$. This means to check the commutativity of

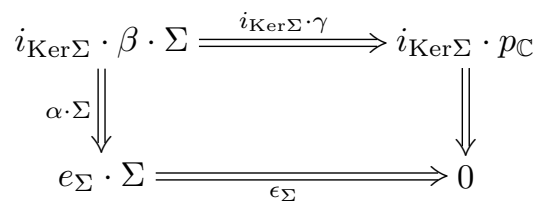

which, by definition of $\gamma_{1}$, amounts to $i_{\operatorname{Ker} \Sigma} \cdot \gamma=\gamma_{1}$.

Corollary 4.4. Let

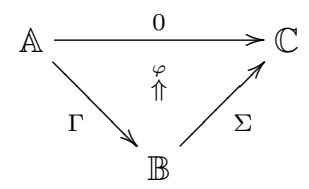

be in $\operatorname{EXT}(\mathbb{C}, \mathbb{A})$. The following conditions are equivalent : 
1) The extension $(\Gamma, \varphi, \Sigma)$ splits ;

2) There exist $S: \mathbb{C} \rightarrow \mathbb{B}$ and $\sigma: S \cdot \Sigma \Rightarrow 1_{\mathbb{C}}$ in $\mathrm{SCG}$;

3) There exist $R: \mathbb{B} \rightarrow \mathbb{A}$ and $\rho: \Gamma \cdot R \Rightarrow 1_{\mathbb{A}}$ in $\mathrm{SCG}$.

Proof. 1$) \Rightarrow 2):$ Given $(\alpha, \beta, \gamma): T(\mathbb{A}, \mathbb{C}) \rightarrow(\Gamma, \varphi, \Sigma)$ in $\operatorname{EXT}(\mathbb{C}, \mathbb{A})$, put $S=i_{\mathbb{C}} \cdot \beta$ and $\sigma: S \cdot \Sigma=i_{\mathbb{C}} \cdot \beta \cdot \Sigma \stackrel{i_{\mathbb{C}} \cdot \gamma}{\Longrightarrow} i_{\mathbb{C}} \cdot p_{\mathbb{C}} \Longrightarrow 1_{\mathbb{C}}$.

2) $\Rightarrow 1)$ : By Lemma 4.3, using that the equivalence $\Gamma_{0}: \mathbb{A} \rightarrow \operatorname{Ker} \Sigma$ induces a biequivalence $\operatorname{EXT}(\mathbb{C}, \mathbb{A}) \simeq \operatorname{EXT}(\mathbb{C}, \operatorname{Ker} \Sigma)$ in which $(\Gamma, \varphi, \Sigma)$ corresponds to $\left(e_{\Sigma}, \epsilon_{\Sigma}, \Sigma\right)$.

1) $\Rightarrow 3) \Rightarrow$ 1) : Similar.

In view of some applications to 2-exact sequences, we need a more precise formulation of Corollary 4.4. For this, fix an extension $(\Gamma, \varphi, \Sigma): \mathbb{A} \rightarrow \mathbb{B} \rightarrow \mathbb{C}$ of $\mathbb{A}$ by $\mathbb{C}$ and consider the category $\operatorname{Split}(\Sigma)$ :

- objects are pairs $\left(S: \mathbb{C} \rightarrow \mathbb{B}, \sigma: S \cdot \Sigma \Rightarrow 1_{\mathbb{C}}\right)$ in SCG ;

- an arrow $\lambda:(S, \sigma) \Rightarrow\left(S^{\prime}, \sigma^{\prime}\right)$ is a 2-cell $\lambda: S \Rightarrow S^{\prime}$ in SCG such that the following diagram commutes

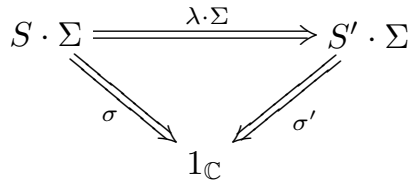

Consider also the trivial extension $T(\mathbb{A}, \mathbb{C})$.

Lemma 4.5. Composition with $i_{\mathbb{C}}: \mathbb{C} \rightarrow \mathbb{A} \times \mathbb{C}$ induces an equivalence

$$
i_{\mathbb{C}} \cdot-: \operatorname{EXT}(\mathbb{C}, \mathbb{A})(T(\mathbb{A}, \mathbb{C}),(\Gamma, \varphi, \Sigma)) \rightarrow \operatorname{Split}(\Sigma)
$$

Proof. Let us describe explicitly the functor $i_{\mathbb{C}} \cdot-$ :

- Given a 1-cell $(\alpha, \beta, \gamma): T(\mathbb{A}, \mathbb{C}) \rightarrow(\Gamma, \varphi, \Sigma)$ in $\operatorname{EXT}(\mathbb{C}, \mathbb{A})$, we obtain an object $\left(\mathbb{C} \stackrel{i_{\mathbb{C}}}{\longrightarrow} \mathbb{A} \times \mathbb{C} \stackrel{\beta}{\longrightarrow} \mathbb{B}, i_{\mathbb{C}} \cdot \gamma: i_{\mathbb{C}} \cdot \beta \cdot \Sigma \Rightarrow i_{\mathbb{C}} \cdot p_{\mathbb{C}}=1_{\mathbb{C}}\right)$ in $\operatorname{Split}(\Sigma)$;

- Given a 2-cell $b:(\alpha, \beta, \gamma) \Rightarrow\left(\alpha^{\prime}, \beta^{\prime}, \gamma^{\prime}\right): T(\mathbb{A}, \mathbb{C}) \rightarrow(\Gamma, \varphi, \Sigma)$ in $\operatorname{EXT}(\mathbb{C}, \mathbb{A})$, then the second condition on $b$ in Definition 2.2 means that $i_{\mathbb{C}} \cdot b:\left(i_{\mathbb{C}} \cdot \beta, i_{\mathbb{C}} \cdot \gamma\right) \Rightarrow$ $\left(i_{\mathbb{C}} \cdot \beta^{\prime}, i_{\mathbb{C}} \cdot \gamma^{\prime}\right)$ is an arrow in $\operatorname{Split}(\Sigma)$.

Now we check that $i_{\mathbb{C}} \cdot-$ is an equivalence :

Faithfulness : let $\bar{b}:(\alpha, \beta, \gamma) \Rightarrow\left(\alpha^{\prime}, \beta^{\prime}, \gamma^{\prime}\right)$ be another 2-cell in $\operatorname{EXT}(\mathbb{C}, \mathbb{A})$. Since $\alpha^{\prime}$ is a natural isomorphism, by the first condition on $b$ and $\bar{b}$ in Definition 2.2 we have that $i_{\mathbb{A}} \cdot b=i_{\mathbb{A}} \cdot \bar{b}$. If, moreover, we assume that $i_{\mathbb{C}} \cdot b=i_{\mathbb{C}} \cdot \bar{b}$, then $b=\bar{b}$.

Fullness : let $\lambda:\left(i_{\mathbb{C}} \cdot \beta, i_{\mathbb{C}} \cdot \gamma\right) \Rightarrow\left(i_{\mathbb{C}} \cdot \beta^{\prime}, i_{\mathbb{C}} \cdot \gamma^{\prime}\right)$ be an arrow in $\operatorname{Split}(\Sigma)$ and consider $i_{\mathbb{A}} \cdot \beta \stackrel{\alpha}{\Longrightarrow} \Gamma \stackrel{\left(\alpha^{\prime}\right)^{-1}}{\Longrightarrow} i_{\mathbb{A}} \cdot \beta^{\prime}$. By the universal property of the coproduct $\mathbb{A} \times \mathbb{C}$, we 
get a unique 2-cell $b: \beta \Rightarrow \beta^{\prime}$ in SCG such that

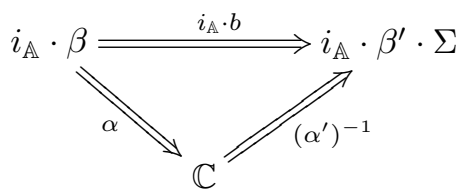

commutes and $i_{\mathbb{C}} \cdot b=\lambda$. It remains to check the commutativity of

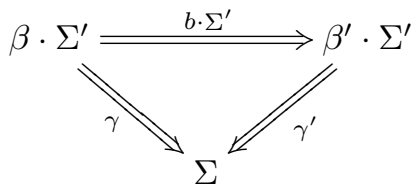

One can do this precomposing with $i_{\mathbb{A}}$ and $i_{\mathbb{C}}$ and using, respectively, that $(\alpha, \beta, \gamma)$ and $\left(\alpha^{\prime}, \beta^{\prime}, \gamma^{\prime}\right)$ are 1-cells in $\operatorname{EXT}(\mathbb{C}, \mathbb{A})$ and the condition on $\lambda$ to be an arrow in $\operatorname{Split}(\Sigma)$.

Essential surjectivity : this is the part already proved in Lemma 4.3 (write everywhere $\mathbb{A}, \varphi$ and $\Gamma$ insted of $\operatorname{Ker} \Sigma, \epsilon_{\Sigma}$ and $e_{\Sigma}$ ). It remains only to check that $\delta:\left(i_{\mathbb{C}} \cdot \beta, i_{\mathbb{C}} \cdot \gamma\right) \Rightarrow(S, \sigma)$ is an arrow in $\operatorname{Split}(\Sigma)$, but this is exactly the second condition on $\gamma$ in the proof of Lemma 4.3.

For later use, let us write explicitly a consequence of Lemma 4.5. Let $(x, L, y):\left(\Gamma_{1}, \varphi_{1}, \Sigma_{1}\right) \rightarrow\left(\Gamma_{2}, \varphi_{2}, \Sigma_{2}\right)$ be a 1-cell in $\operatorname{EXT}(\mathbb{C}, \mathbb{A})$

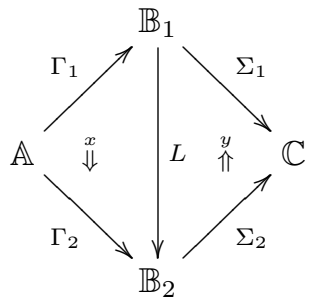

For each pair of 1-cells

$$
\left(\alpha_{1}, \beta_{1}, \gamma_{1}\right): T(\mathbb{A}, \mathbb{C}) \rightarrow\left(\Gamma_{1}, \varphi_{1}, \Sigma_{1}\right) \quad\left(\alpha_{2}, \beta_{2}, \gamma_{2}\right): T(\mathbb{A}, \mathbb{C}) \rightarrow\left(\Gamma_{2}, \varphi_{2}, \Sigma_{2}\right)
$$

in $\operatorname{EXT}(\mathbb{C}, \mathbb{A})$, there is a bijection between 2-cells $b:\left(\alpha_{1}, \beta_{1}, \gamma_{1}\right) \cdot(x, L, y) \Rightarrow$ $\left(\alpha_{2}, \beta_{2}, \gamma_{2}\right)$ in $\operatorname{EXT}(\mathbb{C}, \mathbb{A})$ and arrows

$$
\begin{gathered}
\lambda:\left(\mathbb{C} \stackrel{S_{1}}{\longrightarrow} \mathbb{B}_{1} \stackrel{L}{\longrightarrow} \mathbb{B}_{2}, S_{1} \cdot L \cdot \Sigma_{2} \stackrel{S_{1} \cdot y}{\Longrightarrow} S_{1} \cdot \Sigma_{1} \stackrel{\sigma_{1}}{\Longrightarrow} 1_{\mathbb{C}}\right) \Rightarrow \\
\left(\mathbb{C} \stackrel{S_{2}}{\longrightarrow} \mathbb{B}_{2}, S_{2} \cdot \Sigma_{2} \stackrel{\sigma_{2}}{\longrightarrow} 1_{\mathbb{C}}\right),
\end{gathered}
$$

in $\operatorname{Split}\left(\Sigma_{2}\right)$, where $\left(S_{1}, \sigma_{1}\right)$ and $\left(S_{2}, \sigma_{2}\right)$ correspond to the extensions $\left(\Gamma_{1}, \varphi_{1}, \Sigma_{1}\right)$ and $\left(\Gamma_{2}, \varphi_{2}, \Sigma_{2}\right)$ via the equivalences

$$
\begin{gathered}
i_{\mathbb{C}} \cdot-: \operatorname{EXT}(\mathbb{C}, \mathbb{A})\left(T(\mathbb{A}, \mathbb{C}),\left(\Gamma_{1}, \varphi_{1}, \Sigma_{1}\right)\right) \rightarrow \operatorname{Split}\left(\Sigma_{1}\right) \\
i_{\mathbb{C}} \cdot-: \operatorname{EXT}(\mathbb{C}, \mathbb{A})\left(T(\mathbb{A}, \mathbb{C}),\left(\Gamma_{2}, \varphi_{2}, \Sigma_{2}\right)\right) \rightarrow \operatorname{Split}\left(\Sigma_{2}\right) .
\end{gathered}
$$


This bijection sends $b: \beta_{1} \cdot L \Rightarrow \beta_{2}$ into $\lambda: S_{1} \cdot L=i_{\mathbb{C}} \cdot \beta_{1} \cdot L \stackrel{i_{\mathbb{C}} \cdot b}{\Longrightarrow} i_{\mathbb{C}} \cdot \beta_{2}=S_{2}$.

Clearly, given an extension $(\Gamma, \varphi, \Sigma)$, instead of $\Sigma$ one could consider $\Gamma$ and obtain a dual lemma perfectly analogous to Lemma 4.5. We leave this to the reader.

\section{Pullbacks and pushouts in SCG}

To compare EXT and Hom, as well as to define the 2-dimensional analogue of Baer sum, we need pullbacks and pushouts in SCG (in the sense of bilimits, of course).

Let us start with pushouts. First, we recall the universal property: given two 1-cells $F: \mathbb{A} \rightarrow \mathbb{B}$ and $G: \mathbb{A} \rightarrow \mathbb{C}$ in SCG, their pushout is a diagram in SCG of the form

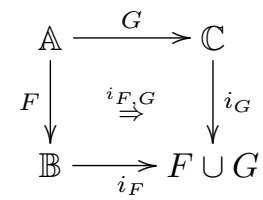

such that, for any other diagram in SCG

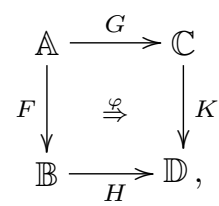

there exist a 1-cell $\varphi^{H, K}: F \cup G \rightarrow \mathbb{D}$ and two 2-cells $\varphi^{H}: i_{F} \cdot \varphi^{H, K} \Rightarrow H, \varphi^{K}: i_{G}$. $\varphi^{H, K} \Rightarrow K$ in SCG such that the following diagram commutes

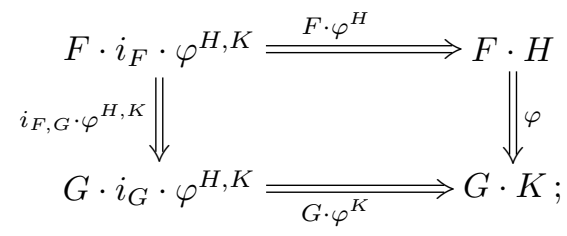

moreover, if $\bar{\varphi}^{H, K}: F \cup G \rightarrow \mathbb{D}, \bar{\varphi}^{H}: i_{F} \cdot \bar{\varphi}^{H, K} \Rightarrow H, \bar{\varphi}^{K}: i_{G} \cdot \bar{\varphi}^{H, K} \Rightarrow K$ satisfy the same condition, then there is a unique 2-cell $\psi: \bar{\varphi}^{H, K} \Rightarrow \varphi^{H, K}$ in SCG such that the following diagrams commute
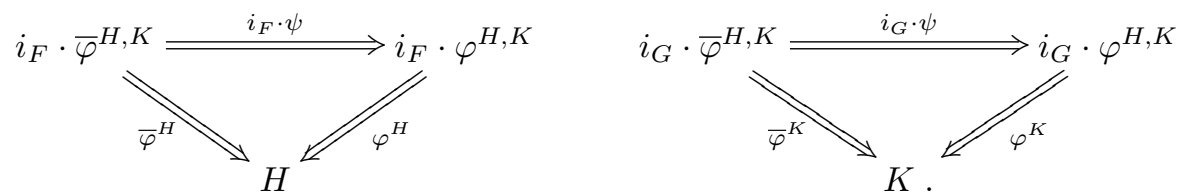

Passing through the description of cokernels in SCG given in $[\mathbf{1 6}]$ and the obvious description of coproducts in SCG, we get an explicit description for pushouts :

- Objects of $F \cup G$ are those of $\mathbb{B} \times \mathbb{C}$;

- A pre-morphism is a triple $(f, A, g):\left(B_{1}, C_{1}\right) \rightarrow\left(B_{2}, C_{2}\right)$, with $A$ an object of $\mathbb{A}$, 
$f: B_{1} \rightarrow F A \otimes B_{2}$ in $\mathbb{B}$ and $g: C_{1} \otimes G A \rightarrow C_{2}$ in $\mathbb{C} ;$

- A morphism is an equivalence class of pre-morphisms :

$(f, A, g),(\bar{f}, \bar{A}, \bar{g}):\left(B_{1}, C_{1}\right) \rightarrow\left(B_{2}, C_{2}\right)$ are equivalent if there exists $\alpha: A \rightarrow \bar{A}$ in $\mathbb{A}$ such that $f \cdot(F \alpha \otimes 1)=\bar{f}$ and $(1 \otimes G \alpha) \cdot \bar{g}=g$; we write $[f, A, g]$ for the equivalence class of a pre-morphism $(f, A, g)$;

- The composition of

$$
\left(B_{1}, C_{1}\right) \stackrel{[f, A, g]}{\longrightarrow}\left(B_{2}, C_{2}\right) \stackrel{\left[f^{\prime}, A^{\prime}, g^{\prime}\right]}{\longrightarrow}\left(B_{3}, C_{3}\right)
$$

is, up to associativity, $\left[f \cdot\left(1 \otimes f^{\prime}\right) \cdot\left(F_{A, A^{\prime}} \otimes 1\right), A \otimes A^{\prime},\left(1 \otimes G_{A, A^{\prime}}^{-1}\right) \cdot(g \otimes 1) \cdot g\right]$;

- The tensor product of

$$
\left(B_{1}, C_{1}\right) \stackrel{[f, A, g]}{\longrightarrow}\left(B_{2}, C_{2}\right) \text { with }\left(B_{1}^{\prime}, C_{1}^{\prime}\right) \stackrel{\left[f^{\prime}, A^{\prime}, g^{\prime}\right]}{\longrightarrow}\left(B_{2}^{\prime}, C_{2}^{\prime}\right)
$$

is $\left[\left(f \otimes f^{\prime}\right) \cdot(1 \otimes \gamma \otimes 1) \cdot\left(1 \otimes 1 \otimes F_{A, A^{\prime}}\right), A \otimes A^{\prime},\left(1 \otimes 1 \otimes G_{A, A^{\prime}}^{-1}\right) \cdot(1 \otimes \gamma \otimes 1) \cdot(g \otimes\right.$ $\left.\left.g^{\prime}\right)\right]:\left(B_{1} \otimes B_{1}^{\prime}, C_{1} \otimes C_{1}^{\prime}\right) \rightarrow\left(B_{2} \otimes B_{2}^{\prime}, C_{2} \otimes C_{2}^{\prime}\right) ;$

- $i_{F}: \mathbb{B} \rightarrow F \cup G$ sends $f: B_{1} \rightarrow B_{2}$ into

$\left[f \cdot l_{B_{2}}^{-1} \cdot\left(F_{I} \otimes 1\right), I,\left(1 \otimes G_{I}^{-1}\right) \cdot l_{I}\right]:\left(B_{1}, I\right) \rightarrow\left(B_{2}, I\right) ;$

- $i_{G}: \mathbb{C} \rightarrow F \cup G$ sends $g: C_{1} \rightarrow C_{2}$ into

$\left[r_{I}^{-1} \cdot\left(1 \otimes F_{I}\right), I,\left(1 \otimes G_{I}^{-1}\right) \cdot r_{C_{1}} \cdot g\right]:\left(I, C_{1}\right) \rightarrow\left(I, C_{2}\right) ;$

- $i_{F, G}: F \cdot i_{F} \Rightarrow G \cdot i_{G}$ is defined, for each $A$ in $\mathbb{A}$, by

$i_{F, G}(A)=\left[r_{F A}^{-1}, A, l_{G A}\right]: i_{F}(F A)=(F A, I) \rightarrow(I, G A)=i_{G}(G A)$;

- $\varphi^{H, K}: F \cup G \rightarrow \mathbb{D}$ sends $[f, A, g]:\left(B_{1}, C_{1}\right) \rightarrow\left(B_{2}, C_{2}\right)$ into

$$
\begin{aligned}
& H\left(B_{1}\right) \otimes K\left(C_{1}\right) \simeq K\left(C_{1}\right) \otimes H\left(B_{1}\right) \\
& \begin{aligned}
& \downarrow 1 \otimes H f \\
& K\left(C_{1}\right) \otimes H\left(F(A) \otimes B_{2}\right) \simeq K\left(C_{1}\right) \otimes H(F(A)) \otimes H\left(B_{2}\right)
\end{aligned} \\
& \downarrow 1 \otimes \varphi_{A} \otimes 1 \\
& K\left(C_{1}\right) \otimes K(G(A)) \otimes H\left(B_{2}\right) \simeq K\left(C_{1} \otimes G(A)\right) \otimes H\left(B_{2}\right) \\
& \downarrow K g \otimes 1 \\
& K\left(C_{2}\right) \otimes H\left(B_{2}\right) \simeq H\left(B_{2}\right) \otimes K\left(C_{2}\right)
\end{aligned}
$$

- $\varphi^{H}: i_{F} \cdot \varphi^{H, K} \Rightarrow H$ is defined, for each $B$ in $\mathbb{B}$, by

$\varphi^{H}(B): \varphi^{H, K}\left(i_{F}(B)\right)=H(B) \otimes K(I) \stackrel{1 \otimes K_{I}^{-1}}{\longrightarrow} H(B) \otimes I \simeq H(B) ;$

- $\varphi^{K}: i_{G} \cdot \varphi^{H, K} \Rightarrow K$ is defined, for each $C$ in $\mathbb{C}$, by

$\varphi^{K}(C): \varphi^{H, K}\left(i_{G}(C)\right)=H(I) \otimes K(C) \stackrel{H_{I}^{-1} \otimes 1}{\longrightarrow} I \otimes K(C) \simeq K(C) ;$

- $\psi: \bar{\varphi}^{H, K} \Rightarrow \varphi^{H, K}$ is defined, for $(B, C)$ in $F \cup G$, by $\psi(B, C): \bar{\varphi}^{H, K}(B, C) \simeq$

$\bar{\varphi}^{H, K}\left(i_{F}(B)\right) \otimes \bar{\varphi}^{H, K}\left(i_{G}(C)\right) \stackrel{\bar{\varphi}^{H}(B) \otimes \bar{\varphi}^{K}(C)}{\longrightarrow} H(B) \otimes K(C)=\varphi^{H, K}(B, C)$.

All what we need about pushouts is the next proposition. 
Proposition 5.1. Consider the following pushout diagram in SCG

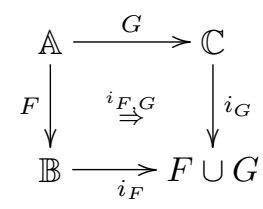

1) If $G$ is faithful, then $i_{F}$ is faithful;

2) If $G$ is full, then $i_{F}$ is full;

3) If $G$ is essentially surjective, then $i_{F}$ is essentially surjective.

Proof. Everything can be checked directly using the previous explicit description. For example, let us prove point 1 . Consider two arrows $f, h: B_{1} \rightarrow B_{2}$ in $\mathbb{B}$ and assume that $i_{F}(f)=i_{F}(h)$ in $F \cup G$. This means that there exists $\alpha: I \rightarrow I$ in $\mathbb{A}$ making commutative the following diagrams
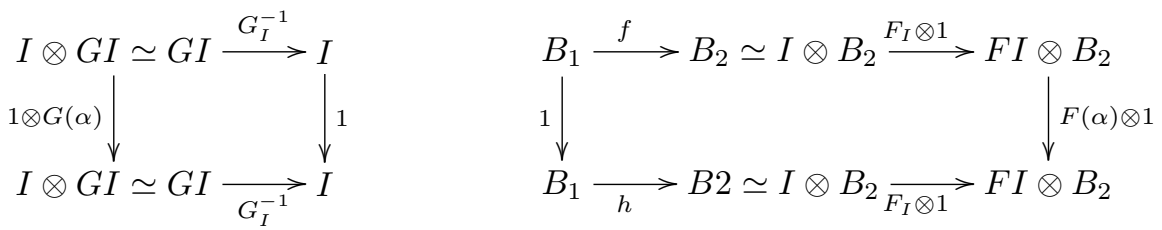

¿From the first equation, we have $G(\alpha)=1_{G I}$. If $G$ is faithful, this implies that $\alpha=1_{I}$. But then, from the second equation, we get $f=h$.

Pullbacks in SCG are easy, in fact they are computed as in the 2-category of groupoids. We fix the notations for future references and we leave to the reader to write the universal property and the explicit description.

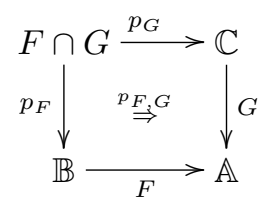

For pullbacks, the dual of Proposition 5.1 holds.

Proposition 5.2. With the previous notations.

1) If $G$ is essentially surjective, then $p_{F}$ is essentially surjective;

2) If $G$ is full, then $p_{F}$ is full;

3) If $G$ is faithful, then $p_{F}$ is faithful.

\section{EXT and Hom}

This section is devoted to the construction of new extensions from a given one, using pullbacks and pushouts. 
Let $\mathbb{C}, \mathbb{D}$ be two symmetric cat-groups; the hom-category $\operatorname{Hom}(\mathbb{D}, \mathbb{C})$ has an obvious structure of symmetric cat-group. This plainly extends to a 2 -functor

$$
\text { Hom }: \mathrm{SCG} \times \mathrm{SCG} \rightarrow \mathrm{SCG}
$$

which reverses the direction of 1-cells in the first variable. Fix now a third symmetric cat-group $\mathbb{A}$ and an extension $E=(\Gamma, \varphi, \Sigma): \mathbb{A} \rightarrow \mathbb{B} \rightarrow \mathbb{C}$ of $\mathbb{A}$ by $\mathbb{C}$.

We need a 2-functor

$$
-\cdot E: \operatorname{Hom}(\mathbb{D}, \mathbb{C}) \rightarrow \operatorname{EXT}(\mathbb{D}, \mathbb{A}),
$$

where we consider $\operatorname{Hom}(\mathbb{D}, \mathbb{C})$ as a 2-category with no non-trivial 2-cells.

For this, consider a 1-cell $G: \mathbb{D} \rightarrow \mathbb{C}$ in SCG and the pullback of $\Sigma$ and $G$, together with the comparison, as in the following diagram

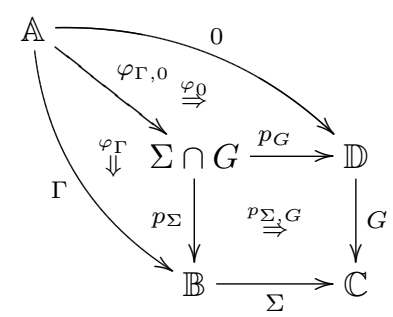

where $\varphi_{\Gamma, 0}, \varphi_{\Gamma}$ and $\varphi_{0}$ make commutative the following diagram

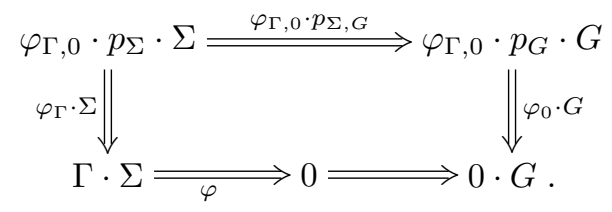

Lemma 6.1. The diagram in SCG

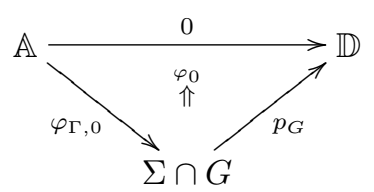

is an extension of $\mathbb{A}$ by $\mathbb{D}$; we denote it by $G \cdot E$.

Proof. From Proposition 5.2, we know that $p_{G}$ is essentially surjective. The fact that

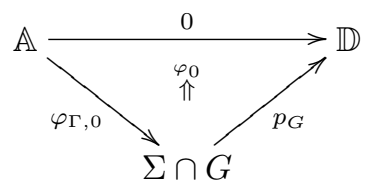

is a kernel of $p_{G}$ can be checked using the universal property of the pullback $\Sigma \cap G$ 
and the universal property of

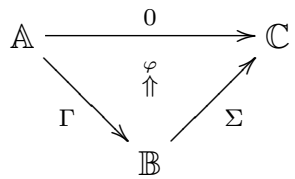

as kernel of $\Sigma$. The detailed proof is long but essentially straightforward, and we omit it.

Consider now another 1-cell $G^{\prime}: \mathbb{D} \rightarrow \mathbb{C}$ in SCG and the extension $G^{\prime} \cdot E$ obtained by the pullback

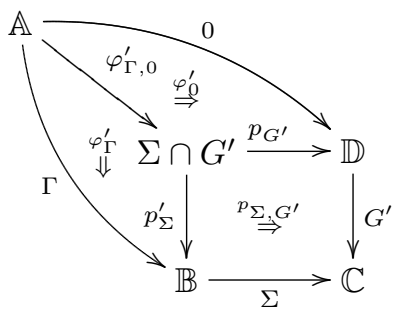

Let $\mu$ : $G \Rightarrow G^{\prime}$ be a 2-cell in SCG ; by the universal property of $\Sigma \cap G^{\prime}$, we obtain a 1-cell $\beta: \Sigma \cap G \rightarrow \Sigma \cap G^{\prime}$ and two 2-cells $s: \beta \cdot p_{\Sigma}^{\prime} \Rightarrow p_{\Sigma}, \gamma: \beta \cdot p_{G^{\prime}} \Rightarrow p_{G}$ in SCG such that the following diagram commutes

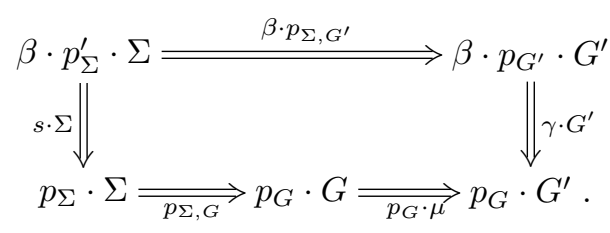

We are looking for a 1-cell in $\operatorname{EXT}(\mathbb{D}, \mathbb{A})$ induced by $\mu$ :

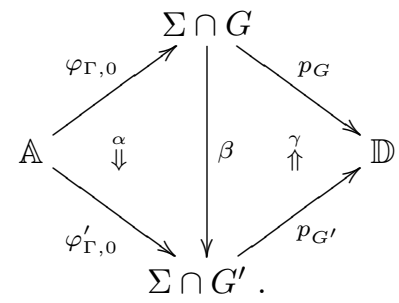

As far as $\alpha$ is concerned, we have

$$
\begin{gathered}
\alpha_{\mathbb{D}}: \varphi_{\Gamma, 0} \cdot \beta \cdot p_{G^{\prime}} \stackrel{\varphi_{\Gamma, 0} \cdot \gamma}{\Longrightarrow} \varphi_{\Gamma, 0} \cdot p_{G} \stackrel{\varphi_{0}}{\longrightarrow} 0 \stackrel{\left(\varphi_{0}^{\prime}\right)^{-1}}{\longrightarrow} \varphi_{\Gamma, 0}^{\prime} \cdot p_{G^{\prime}} \\
\alpha_{\mathbb{B}}: \varphi_{\Gamma, 0} \cdot \beta \cdot p_{\Sigma}^{\prime} \stackrel{\varphi_{\Gamma, 0} \cdot s}{\longrightarrow} \varphi_{\Gamma, 0} \cdot p_{\Sigma} \stackrel{\varphi_{\Gamma}}{\longrightarrow} 0 \stackrel{\left(\varphi_{\Gamma}^{\prime}\right)^{-1}}{\longrightarrow} \varphi_{\Gamma, 0}^{\prime} \cdot p_{\Sigma}^{\prime} .
\end{gathered}
$$


Since

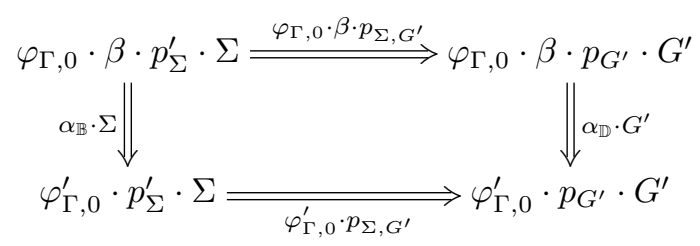

commutes, there is a unique 2-cell $\alpha: \varphi_{\Gamma, 0} \cdot \beta \Rightarrow \varphi_{\Gamma, 0}^{\prime}$ such that $\alpha \cdot p_{G^{\prime}}=\alpha_{\mathbb{D}}$ and $\alpha \cdot p_{\Sigma}^{\prime}=\alpha_{\mathbb{B}}$.

Lemma 6.2. With the previous notations,

$$
(\alpha, \beta, \gamma):\left(\varphi_{\Gamma, 0}, \varphi_{0}, p_{G}\right) \rightarrow\left(\varphi_{\Gamma, 0}^{\prime}, \varphi_{0}^{\prime}, p_{G}^{\prime}\right)
$$

is a 1-cell in $\operatorname{EXT}(\mathbb{D}, \mathbb{A})$; we denote it by $\mu \cdot E$.

Proof. The condition to be a 1 -cell in $\operatorname{EXT}(\mathbb{D}, \mathbb{A})$ is precisely the equation $\alpha \cdot p_{G^{\prime}}=$ $\alpha_{\mathbb{D}}$.

We pass now to the dual construction, involving pushouts. For this, consider an extension $E=(\Gamma, \varphi, \Sigma): \mathbb{A} \rightarrow \mathbb{B} \rightarrow \mathbb{C}$ in SCG and fix a 1-cell $F: \mathbb{A} \rightarrow \mathbb{D}$ in SCG. Consider also the pushout of $F$ and $\Gamma$ and the factorization as in the following diagram

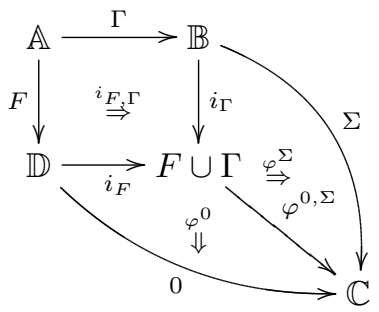

with $\varphi^{0, \Sigma}, \varphi^{0}$ and $\varphi^{\Sigma}$ making commutative the diagram

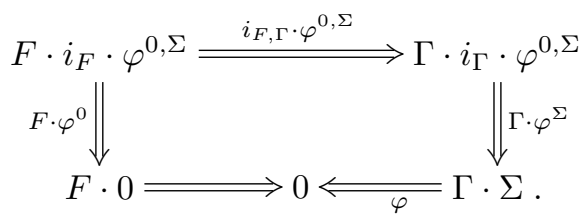

Lemma 6.3. The diagram in $\mathrm{SCG}$

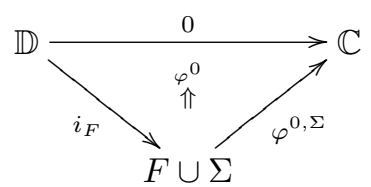

is an extension of $\mathbb{D}$ by $\mathbb{C} ;$ we denote it by $E \cdot F$. 
Proof. From Proposition 5.1, we know that $i_{F}$ is faithful. Moreover, one can check directly the universal property of the cokernel

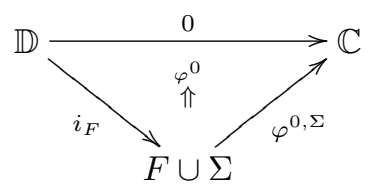

using the universal property of the pushout $F \cup \Gamma$ and that of the cokernel

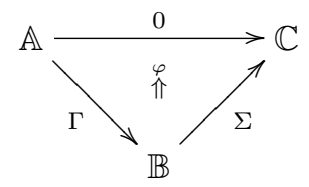

The construction of the extension $E \cdot F$ of Lemma 6.3 fits into a 2-functor

$$
-\cdot F: \operatorname{EXT}(\mathbb{C}, \mathbb{A}) \rightarrow \operatorname{EXT}(\mathbb{C}, \mathbb{D})
$$

Let us describe it in detail.

Consider another extension $E^{\prime}=\left(\Gamma^{\prime}, \varphi^{\prime}, \Sigma^{\prime}\right): \mathbb{A} \rightarrow \mathbb{B}^{\prime} \rightarrow \mathbb{C}$ with the corresponding factorization

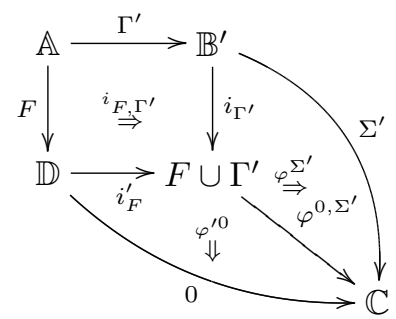

and let $(\alpha, \beta, \gamma):(\Gamma, \varphi, \Sigma) \rightarrow\left(\Gamma^{\prime}, \varphi^{\prime}, \Sigma^{\prime}\right)$ be a 1-cell in $\operatorname{EXT}(\mathbb{C}, \mathbb{A})$. By the universal property of the pushout $F \cup \Gamma$, we get $\beta_{F}: F \cup \Gamma \rightarrow F \cup \Gamma^{\prime}, \alpha_{F}: i_{F} \cdot \beta_{F} \Rightarrow$ $i_{F}^{\prime}, \delta_{F}: i_{\Gamma} \cdot \beta_{F} \Rightarrow \beta \cdot i_{\Gamma^{\prime}}$ making commutative the following diagram

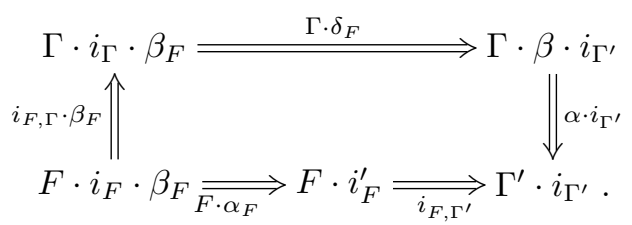


Consider now the 2-cells

$$
\begin{aligned}
& \gamma_{\mathbb{B}}: i_{\Gamma} \cdot \beta_{F} \cdot \varphi^{0, \Sigma^{\prime}} \stackrel{\delta_{F} \cdot \varphi^{0, \Sigma^{\prime}}}{\Longrightarrow} \beta \cdot i_{\Gamma^{\prime}} \cdot \varphi^{0, \Sigma^{\prime}} \stackrel{\beta \cdot \varphi^{\Sigma^{\prime}}}{\longrightarrow} \beta \cdot \Sigma^{\prime} \stackrel{\gamma}{\longrightarrow} \|_{\|_{\left(\varphi^{\Sigma}\right)^{-1}}} \\
& i_{\Gamma} \cdot \varphi^{0, \Sigma} \\
& \gamma_{\mathbb{D}}: i_{F} \cdot \beta_{F} \cdot \varphi^{0, \Sigma^{\prime}} \stackrel{\alpha_{F} \cdot \varphi^{0, \Sigma^{\prime}}}{=} i_{F}^{\prime} \cdot \varphi^{0, \Sigma^{\prime}} \stackrel{\varphi^{\prime 0}}{=} 0 \stackrel{\left(\varphi^{0}\right)^{-1}}{\Longrightarrow} i_{F} \cdot \varphi^{0, \Sigma} .
\end{aligned}
$$

Since they are compatible with $i_{F, \Gamma}$, there exists a unique $\gamma_{F}: \beta_{F} \cdot \varphi^{0, \Sigma^{\prime}} \Rightarrow \varphi^{0, \Sigma}$ such that $i_{F} \cdot \gamma_{F}=\gamma_{\mathbb{D}}$ and $i_{\Gamma} \cdot \gamma_{F}=\gamma_{\mathbb{B}}$. In particular,

$$
\left(\alpha_{F}, \beta_{F}, \gamma_{F}\right):\left(i_{F}, \varphi^{0}, \varphi^{0, \Sigma}\right) \rightarrow\left(i_{F}^{\prime}, \varphi^{\prime 0}, \varphi^{0, \Sigma^{\prime}}\right)
$$

is a 1-cell in $\operatorname{EXT}(\mathbb{C}, \mathbb{D})$.

Starting from another 1-cell $\left(\alpha^{\prime}, \beta^{\prime}, \gamma^{\prime}\right):(\Gamma, \varphi, \Sigma) \rightarrow\left(\Gamma^{\prime}, \varphi^{\prime}, \Sigma^{\prime}\right)$ in $\operatorname{EXT}(\mathbb{C}, \mathbb{A})$, repeat the previous argument. Consider also a 2-cell $b:(\alpha, \beta, \gamma) \Rightarrow\left(\alpha^{\prime}, \beta^{\prime}, \gamma^{\prime}\right)$. Because of the first condition in the definition of 2-cell, the universal property of the pushout $F \cup \Gamma$ gives a unique 2-cell $b_{F}: \beta_{F} \Rightarrow \beta_{F}^{\prime}$ making commutative the following diagram
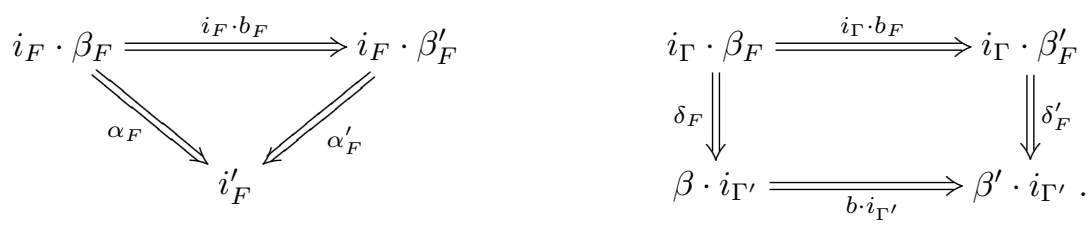

To have a 2-cell $b_{F}:\left(\alpha, \beta_{F}, \gamma_{F}\right) \Rightarrow\left(\alpha_{F}^{\prime}, \beta_{F}^{\prime}, \gamma_{F}^{\prime}\right)$ in $\operatorname{EXT}(\mathbb{C}, \mathbb{D})$, it remains to check the commutativity of

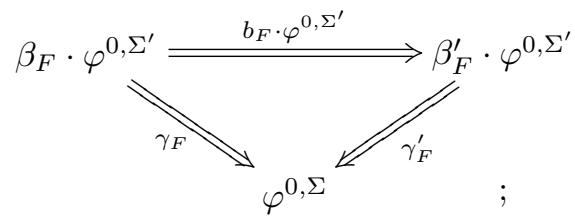

this can be done, via the definition of $\gamma_{F}$, using the second condition on the 2-cell $b$ and the two equations satisfied by $b_{F}$.

To end this section, let us summarize the situation just discussed, as well as the dual situation.

Proposition 6.4. Consider two 1-cells $F: \mathbb{A} \rightarrow \mathbb{D}, G: \mathbb{D} \rightarrow \mathbb{C}$ in $\mathrm{SCG}$ and an extension $E=(\Gamma, \varphi, \Sigma): \mathbb{A} \rightarrow \mathbb{B} \rightarrow \mathbb{C}$. The pullback construction gives two 2- 
functors

$$
\begin{aligned}
& -\cdot E: \operatorname{Hom}(\mathbb{D}, \mathbb{C}) \longrightarrow \operatorname{EXT}(\mathbb{D}, \mathbb{A}) \\
& G \cdot-: \operatorname{EXT}(\mathbb{C}, \mathbb{A}) \longrightarrow \operatorname{EXT}(\mathbb{D}, \mathbb{A}) .
\end{aligned}
$$

The pushout construction gives two 2-functors

$$
\begin{aligned}
& E \cdot-: \operatorname{Hom}(\mathbb{A}, \mathbb{D}) \longrightarrow \operatorname{EXT}(\mathbb{C}, \mathbb{D}) \\
& -\cdot F: \operatorname{EXT}(\mathbb{C}, \mathbb{A}) \longrightarrow \operatorname{EXT}(\mathbb{C}, \mathbb{D}) .
\end{aligned}
$$

\section{First application}

As a first, simple application, let us show that EXT measures if a 1-cell can be lifted (or extended).

Proposition 7.1. Consider a 1-cell $G: \mathbb{D} \rightarrow \mathbb{C}$ in $\mathrm{SCG}$ and an extension $E=$ $(\Gamma, \varphi, \Sigma): \mathbb{A} \rightarrow \mathbb{B} \rightarrow \mathbb{C}$ of $\mathbb{A}$ by $\mathbb{C}$. There exist $\bar{G}: \mathbb{D} \rightarrow \mathbb{B}$ and $\psi: \bar{G} \cdot \Sigma \Rightarrow G$ in SCG if and only if the extension $G \cdot E$ in $\operatorname{EXT}(\mathbb{D}, \mathbb{A})$ splits.

Proof. If $G \cdot E$ splits, there is a section $S: \mathbb{D} \rightarrow \Sigma \cap G, \sigma: S \cdot p_{G} \Rightarrow 1_{\mathbb{D}}$ of $p_{G}$ in SCG. Then one can put $\bar{G}=S \cdot p_{\Sigma}$ and

$$
\psi: S \cdot p_{\Sigma} \cdot \Sigma \stackrel{S \cdot p_{\Sigma, G}}{\Longrightarrow} S \cdot p_{G} \cdot G \stackrel{\sigma \cdot G}{\Longrightarrow} 1_{\mathbb{D}} \cdot G=G .
$$

Conversely, consider

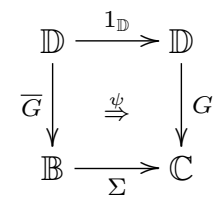

By the universal property of the pullback $\Sigma \cap G$, we get

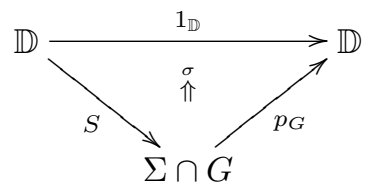

in $\mathrm{SCG}$

We state the dual proposition, whose proof is left to the reader.

Proposition 7.2. Consider a 1-cell $F: \mathbb{A} \rightarrow \mathbb{D}$ in SCG and an extension $E=$ $(\Gamma, \varphi, \Sigma): \mathbb{A} \rightarrow \mathbb{B} \rightarrow \mathbb{C}$ of $\mathbb{A}$ by $\mathbb{C}$. There exist $\bar{F}: \mathbb{B} \rightarrow \mathbb{D}$ and $\psi: \Gamma \cdot \bar{F} \Rightarrow F$ in $\mathrm{SCG}$ if and only if the extension $E \cdot F$ in $\operatorname{EXT}(\mathbb{C}, \mathbb{D})$ splits. 


\section{Proper classes of extensions}

As explained in the Introduction, we define now the notion of proper class of extensions, in order to make results of Sections 10, 11 and 13 available for some special classes of extensions.

The next definition extends Definition 2.2. It is useful to define proper classes, and also to define Baer sum (see Section 13).

Definition 8.1. Consider two extensions $E=(\Gamma, \varphi, \Sigma)$ and $E^{\prime}=\left(\Gamma^{\prime}, \varphi^{\prime}, \Sigma^{\prime}\right)$ in SCG
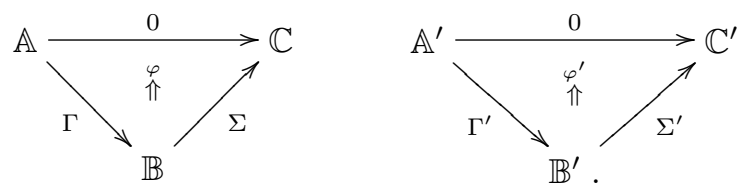

1) A 1-cell $(F, \alpha, \beta, \gamma, G): E \rightarrow E^{\prime}$ is pictured in the following diagram

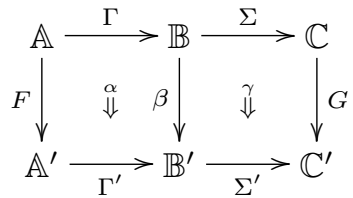

with $F, \alpha, \beta, \gamma, G$ in SCG and such that the following diagram commutes

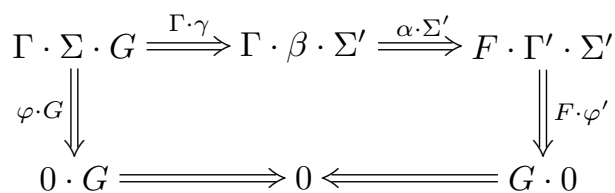

2) If $(F, \alpha, \beta, \gamma, G),\left(F^{\prime}, \alpha^{\prime}, \beta^{\prime}, \gamma^{\prime}, G^{\prime}\right): E \rightarrow E^{\prime}$ are two 1-cells, a 2-cell

$$
(x, y, z):(F, \alpha, \beta, \gamma, G) \Rightarrow\left(F^{\prime}, \alpha^{\prime}, \beta^{\prime}, \gamma^{\prime}, G^{\prime}\right)
$$

is a triple $x: F \Rightarrow F^{\prime}, y: \beta \Rightarrow \beta^{\prime}, z: G \Rightarrow G^{\prime}$ of 2-cells in SCG making commutative the following diagrams
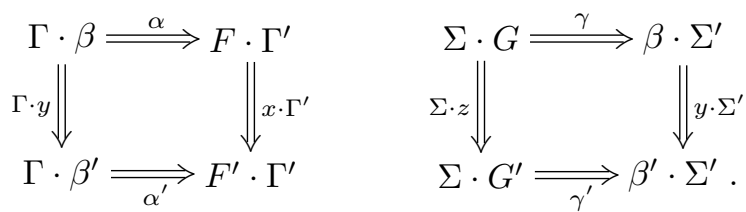

Observe that 2-cells are invertible, and that a 1-cell $(F, \alpha, \beta, \gamma, G)$ is invertible as soon as $F$ and $G$ are equivalences. The proof is similar to that of Proposition 2.8.

Definition 8.2. A proper class $\mathcal{P}$ of extensions is the assignement, for every pair of symmetric cat-groups $\mathbb{A}$ and $\mathbb{C}$, of a full (at the level of 1-cells and 2-cells) sub2-category $\mathcal{P}(\mathbb{C}, \mathbb{A})$ of $\operatorname{EXT}(\mathbb{C}, \mathbb{A})$ in such a way that:

1) The trivial extension $T(\mathbb{A}, \mathbb{C})$ is in $\mathcal{P}(\mathbb{C}, \mathbb{A})$; 
2) If $E \in \mathcal{P}(\mathbb{C}, \mathbb{A}), E^{\prime} \in \operatorname{EXT}\left(\mathbb{C}^{\prime}, \mathbb{A}^{\prime}\right)$ and $(F, \alpha, \beta, \gamma, G): E \rightarrow E^{\prime}$ is an equivalence (in the sense of Definition 8.1), then $E^{\prime} \in \mathcal{P}\left(\mathbb{C}^{\prime}, \mathbb{A}^{\prime}\right)$;

3) The 2-functors of Proposition 6.4 restrict to $\mathcal{P}$

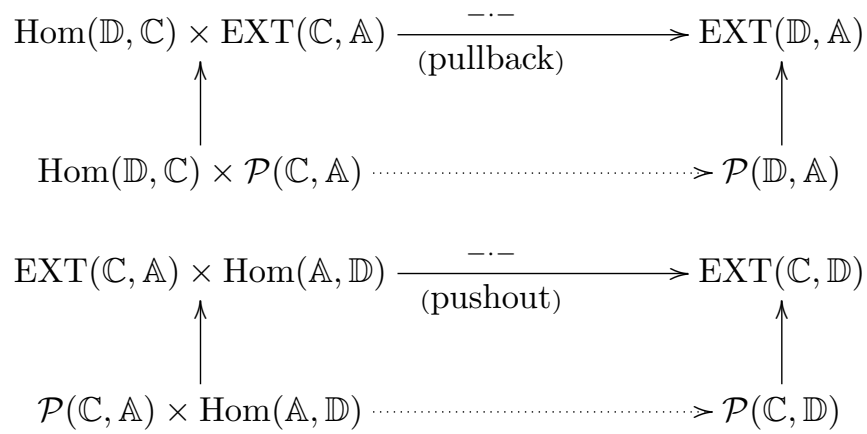

To prepare the study of injective and projective symmetric cat-groups, let us observe that a proper class $\mathcal{P}$ of extensions gives rise to two classes of morphisms in SCG.

Definition 8.3. Let $\mathcal{P}$ be a proper class of extensions.

1) $A$ morphism $\Gamma: \mathbb{A} \rightarrow \mathbb{B}$ in $\mathrm{SCG}$ is a $\mathcal{P}$-mono if there exist $\Sigma: \mathbb{B} \rightarrow \mathbb{C}$ and $\varphi: \Gamma \cdot \Sigma \Rightarrow 0$ in $\mathrm{SCG}$ such that $(\Gamma, \varphi, \Sigma) \in \mathcal{P}(\mathbb{C}, \mathbb{A})$. Equivalently, $\Gamma$ is a $\mathcal{P}$-mono if $\left(\Gamma, \pi_{\Gamma}, P_{\Gamma}\right) \in \mathcal{P}(\operatorname{Coker} \Gamma, \mathbb{A})$.

2) $A$ morphism $\Sigma: \mathbb{B} \rightarrow \mathbb{C}$ in $\mathrm{SCG}$ is a $\mathcal{P}$-epi if there exist $\Gamma: \mathbb{A} \rightarrow \mathbb{B}$ and $\varphi: \Gamma \cdot \Sigma \Rightarrow 0$ in $\mathrm{SCG}$ such that $(\Gamma, \varphi, \Sigma) \in \mathcal{P}(\mathbb{C}, \mathbb{A})$. Equivalently, $\Sigma$ is a $\mathcal{P}$-epi if $\left(e_{\Sigma}, \epsilon_{\Sigma}, \Sigma\right) \in \mathcal{P}(\mathbb{C}, \operatorname{Ker} \Sigma)$.

The classes $\mathcal{P}$-mono and $\mathcal{P}$-epi inherit some good 2-categorical property from those of the proper class $\mathcal{P}$.

Proposition 8.4. Let $\mathcal{P}$ be a proper class of extensions.

0) For any $\mathbb{A} \in \mathrm{SCG}$, the canonic morphism $\mathbf{1} \rightarrow \mathbb{A}$ is a $\mathcal{P}$-mono;

0') For any $\mathbb{A} \in \mathrm{SCG}$, the canonic morphism $\mathbb{A} \rightarrow \mathbf{1}$ is a $\mathcal{P}$-epi;

1) Equivalences $\subseteq \mathcal{P}$-mono $\subseteq$ faithful functors;

1') Equivalences $\subseteq \mathcal{P}$-epi $\subseteq$ essentially surjective functors;

2) $\mathcal{P}$-monos are stable under composition with equivalences and under natural isomorphisms;

2') $\mathcal{P}$-epis are stable under composition with equivalences and under natural isomorphisms;

3) $\mathcal{P}$-monos are stable under pushouts;

3') $\mathcal{P}$-epis are stable under pullbacks;

4) Given $F: \mathbb{A} \rightarrow \mathbb{E}, H: \mathbb{E} \rightarrow \mathbb{B}$ in $\mathrm{SCG}$, if $F \cdot H$ is a $\mathcal{P}$-mono then $F$ is a $\mathcal{P}$-mono;

$\left.4^{\prime}\right)$ Given $L: \mathbb{B} \rightarrow \mathbb{E}, G: \mathbb{E} \rightarrow \mathbb{C}$ in $\mathrm{SCG}$, if $L \cdot G$ is a $\mathcal{P}$-epi then $G$ is a $\mathcal{P}$-epi. 
The non trivial points are 4 and 4'. Their proof is based on the next lemma

\section{Lemma 8.5.}

1) Consider the following diagram in SCG, where $\bar{H}$ and $\bar{h}$ are induced by the universal property of Coker $F$,

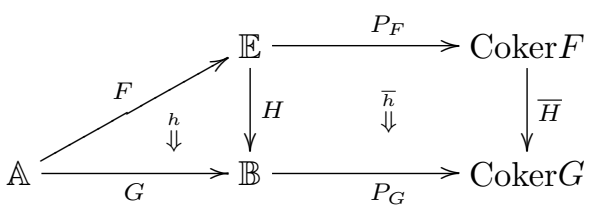

If $G$ is faithful, then the square is a pullback.

2) Consider the following diagram in SCG, where $\bar{L}$ and $\bar{l}$ are induced by the universal property of $\mathrm{Ker} G$,

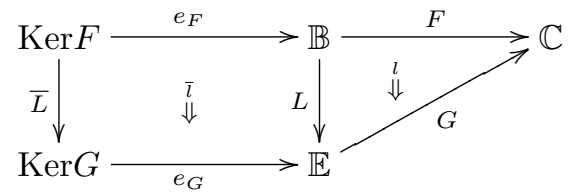

If $F$ is essentially surjective, then the square is a pushout.

Proof. Let us prove part 2, the proof of part 1 is dual. Observe that, since $F$ is essentially surjective, $\left(e_{F}, \epsilon_{F}, F\right): \operatorname{Ker} F \rightarrow \mathbb{B} \rightarrow \mathbb{C}$ and $\left(e_{G}, \epsilon_{G}, G\right): \operatorname{Ker} G \rightarrow \mathbb{E} \rightarrow \mathbb{C}$ are extensions. Consider now the factorization through the pushout (Section 5) and the pushout extension (Section 6) as in the following diagrams
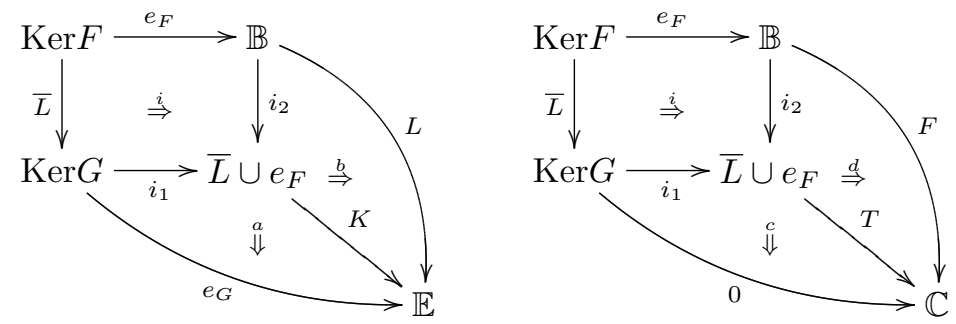

Using the universal property of the pushout $\bar{L} \cup e_{F}$, one can complete the following diagram with a 2-cell $e: K \cdot G \Rightarrow T$ and check that it is a 1-cell between extensions

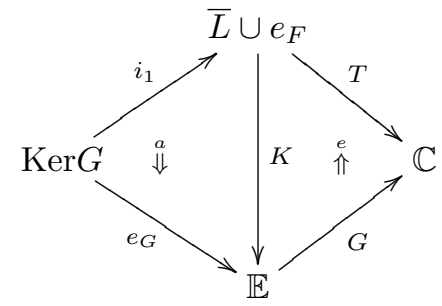

By Proposition 2.8, the proof is complete. 
The proof of 8.4.4 and 8.4.4' is now obvious. Consider the diagram of 8.5.2 with $F=L \cdot G$ and $l=L \cdot G$. Since $F$ is a $\mathcal{P}$-epi, the extension $\left(e_{F}, \epsilon_{F}, F\right)$ is in $\mathcal{P}(\mathbb{C}, \operatorname{Ker} F)$. But $\left(e_{G}, \epsilon_{G}, G\right)$ is the pushout extension of $\left(e_{F}, \epsilon_{F}, F\right)$ along $\bar{L}$, so that it is in $\mathcal{P}(\mathbb{C}, \operatorname{Ker} G)$, that is $G$ is a $\mathcal{P}$-epi. The proof of 8.4.4 is similar.

We have constructed the classes $\mathcal{P}$-mono and $\mathcal{P}$-epi starting from a proper class of extensions. It is possible to work in the opposite direction.

Proposition 8.6. Let $\mathcal{M}$ and $\mathcal{E}$ be two classes of morphisms in SCG. Assume that $\mathcal{M}$ satisfies conditions 0,1,2, 3 and 4 of Proposition 8.4 and that $\mathcal{E}$ satisfies conditions 0', 1', 2', 3' and 4' of Proposition 8.4. Define $\mathcal{P}(\mathbb{C}, \mathbb{A})$ as the full sub-2category of $\operatorname{EXT}(\mathbb{C}, \mathbb{A})$ of those extensions $(\Gamma, \varphi, \Sigma): \mathbb{A} \rightarrow \mathbb{B} \rightarrow \mathbb{C}$ such that $\Gamma \in \mathcal{M}$ and $\Sigma \in \mathcal{E}$. Then $\mathcal{P}$ is a proper class of extensions.

Proof. Let us check, for example, that if $E=(\Gamma, \varphi, \Sigma): \mathbb{A} \rightarrow \mathbb{B} \rightarrow \mathbb{C}$ is in $\mathcal{P}(\mathbb{C}, \mathbb{A})$, then for any $G: \mathbb{D} \rightarrow \mathbb{C}$ the pullback extension $G \cdot E$ is in $\mathcal{P}(\mathbb{D}, \mathbb{A})$. Consider the diagram defining $G \cdot E$ (Section 6 )

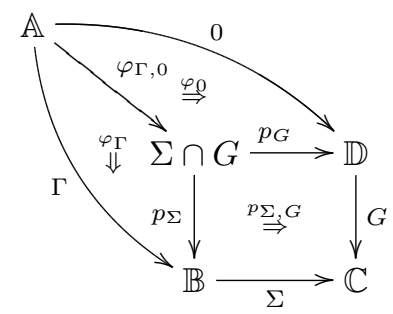

By condition $3, p_{G}$ is in $\mathcal{E}$; by condition 4 and the fact that $\mathcal{M}$ is closed under natural isomorphisms, we have that $\varphi_{\Gamma, 0}$ is in $\mathcal{E}$.

Finally, let us observe that the class $\mathcal{E}$ alone suffices to construct a proper class of extensions.

Proposition 8.7. Let $\mathcal{E}$ be a class of morphisms in SCG satisfying conditions 0', 1', 2', 3' and 4' of Proposition 8.4. Define the class $\mathcal{M}$ as the class of those faithful morphisms $\Gamma: \mathbb{A} \rightarrow \mathbb{B}$ in $\mathrm{SCG}$ whose cokernel is in $\mathcal{E}$ (equivalently, which are the kernel of some morphism $\Sigma: \mathbb{B} \rightarrow \mathbb{C}$ in $\mathcal{E}$ ). Then $\mathcal{M}$ satisfies conditions 0, 1, 2, 3 and 4 of Proposition 8.4.

Proof. Let us check condition 3. Consider an extension $(\Gamma, \varphi, \Sigma): \mathbb{A} \rightarrow \mathbb{B} \rightarrow \mathbb{C}$ with $\Gamma$ in $\mathcal{E}$. Consider also the pushout extension along any $F: \mathbb{A} \rightarrow \mathbb{D}$ in SCG (Section 6)

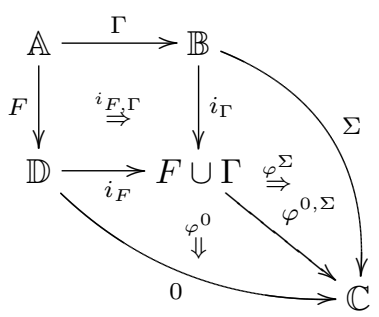


Since $\Gamma \in \mathcal{M}$, then $\Sigma \in \mathcal{E}$. By condition $4^{\prime}, \varphi^{0, \Sigma} \in \mathcal{E}$ and then its kernel $i_{\Sigma}$ is in $\mathcal{M}$. As far as condition 4 is concerned, it follows from part 1 of Lemma 8.5 and the assumption that $\mathcal{E}$ is stable under pullbacks.

Dually, the class $\mathcal{E}$ can be constructed by the class $\mathcal{M}$, so that the class $\mathcal{M}$ alone suffices to get a proper class of extensions.

\section{Examples of proper classes}

Example 9.1. Clearly, EXT is a proper class of extensions. In this case, $\mathcal{P}$-epis are the essentially surjective functors and $\mathcal{P}$-monos are the faithful functors.

Example 9.2. Define an extension $(\Gamma, \varphi, \Sigma): \mathbb{A} \rightarrow \mathbb{B} \rightarrow \mathbb{C}$ to be a $\mathcal{G}$-extension if $\Sigma: \mathbb{B} \rightarrow \mathbb{C}$ is a Grothendieck fibration. This class is not proper (for example because Grothendieck fibrations are not stable under natural isomorphisms). Moreover, the smallest proper class containing $\mathcal{G}$ is EXT. Indeed, if $\Sigma: \mathbb{B} \rightarrow \mathbb{C}$ is any functor between groupoids, the standard (bi)pullback of $\Sigma$ along the identity on $\mathbb{C}$ is a Grothendieck fibration equivalent to $\Sigma$.

Example 9.3. We arrive now to the example which motivates the abstract approach of Section 8 .

Definition 9.4. An extension $(\Gamma, \varphi, \Sigma): \mathbb{A} \rightarrow \mathbb{B} \rightarrow \mathbb{C}$ is an $\mathcal{F}$-extension if there exist a functor $S: \mathbb{C} \rightarrow \mathbb{B}$ and a natural transformation $\sigma: S \cdot \Sigma \Rightarrow 1_{\mathbb{C}}$.

Let us insist on the fact $\mathcal{F}$-extensions are not trivial, because we do not require $S$ and $\sigma$ to be monoidal. The fact that $\mathcal{F}$ is a proper class is quite obvious. We only point out that the stability under pullbacks is due to the fact (already observed) that pullbacks in SCG are computed as in the 2-category of groupoids, so that a functorial section for $\Sigma: \mathbb{B} \rightarrow \mathbb{C}$ gives rise to a functorial section for any pullback of $\Sigma$. The last section of this paper is devoted to a cohomological classification of $\mathcal{F}$-extensions.

We have defined the class $\mathcal{F}$ using $\mathcal{F}$-epis. We want now to characterize $\mathcal{F}$-monos.

Proposition 9.5. Let $(\Gamma, \varphi, \Sigma): \mathbb{A} \rightarrow \mathbb{B} \rightarrow \mathbb{C}$ be an extension. The following conditions are equivalent:

1) $(\Gamma, \varphi, \Sigma)$ is an $\mathcal{F}$-extension;

2) There exists a functor $R: \mathbb{B} \rightarrow \mathbb{A}$ and a coherent (w.r.t. the monoidal structure of $\Gamma$ ) natural transformation

$$
\nu=\left(\nu_{B, A}: R(B \otimes \Gamma(A)) \rightarrow R(B) \otimes A\right)_{(B, A) \in \mathbb{B} \times \mathbb{A}}
$$

To prove Proposition 9.5, we need the following lemma, which says that the section $(S, \sigma)$ can be normalized, and that the pair $R, \nu$ induces a normalized retraction for $\Gamma$. 


\section{Lemma 9.6.}

1) Let $\Sigma: \mathbb{B} \rightarrow \mathbb{C}, S: \mathbb{C} \rightarrow \mathbb{B}, \sigma: S \cdot \Sigma \Rightarrow 1_{\mathbb{C}}$ be as in Definition 9.4; there is a functor $\bar{S}: \mathbb{C} \rightarrow \mathbb{B}$, a natural transformation $\bar{\sigma}: \bar{S} \cdot \Sigma \Rightarrow 1_{\mathbb{C}}$ and a morphism $\bar{S}_{I}: I \rightarrow \bar{S}(I)$ such that the following diagram commutes

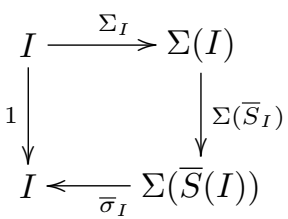

2) Let $\Gamma: \mathbb{A} \rightarrow \mathbb{B}, R: \mathbb{B} \rightarrow \mathbb{A}$ and $\nu$ be as in Proposition 9.5; there is a functor $\bar{R}: \mathbb{B} \rightarrow \mathbb{A}$, a natural transformation $\bar{\rho}: \Gamma \cdot \bar{R} \Rightarrow 1_{\mathbb{A}}$ and a morphism $\bar{R}_{I}: I \rightarrow$ $\bar{R}(I)$ such that the following diagram commutes

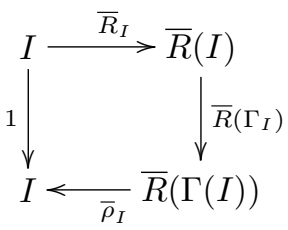

Proof. 1) Define

$$
\begin{gathered}
\bar{S}: \quad f: X \rightarrow Y \mapsto S(f) \otimes 1: S(X) \otimes S(I)^{*} \rightarrow S(Y) \otimes S(I)^{*} \\
\bar{S}_{I}=\eta_{S(I)}: I \rightarrow S(I) \otimes S(I)^{*}=\bar{S}(I) \\
\bar{\sigma}_{X}: \Sigma(\bar{S}(X)) \simeq \Sigma(S(X)) \otimes \Sigma(S(I))^{*} \stackrel{\sigma_{X} \otimes\left(\sigma_{I}^{-1}\right)^{*}}{\longrightarrow} X \otimes I^{*} \simeq X \otimes I \simeq X
\end{gathered}
$$

2) Define $\bar{R}(X)=R(X) \otimes R(I)^{*}$ and $\bar{R}_{I}=\eta_{R(I)}: I \rightarrow R(I) \otimes R(I)^{*}=\bar{R}(I)$. We get a natural transformation $\bar{\nu}_{B, A}=\nu_{B, A} \cdot(1 \otimes \gamma): \bar{R}(B \otimes \Gamma(A))=R(B \otimes \Gamma(A)) \otimes$ $R(I)^{*} \rightarrow R(B) \otimes A \otimes R(I)^{*} \rightarrow R(B) \otimes R(I)^{*} \otimes A=\bar{R}(B) \otimes A$. Finally, we have $\rho_{A}=\bar{\nu}_{I, A} \cdot \bar{R}_{I}^{-1}: \bar{R}(\Gamma(A)) \simeq \bar{R}(I \otimes \Gamma(A)) \rightarrow \bar{R}(I) \otimes A \rightarrow I \otimes A \simeq A$.

Now we can come back to the proof of Proposition 9.5.

Proof. 1) $\Rightarrow 2):$ Since $\Gamma_{0}: \mathbb{A} \rightarrow \operatorname{Ker} \Sigma$ is an equivalence, to prove condition 2) we look for a functor $R: \mathbb{B} \rightarrow \operatorname{Ker} \Sigma$. Let $B$ be an object of $\mathbb{B}$, we define

$$
R(B)=\left(B \otimes S(\Sigma(B))^{*}, R_{B}\right),
$$

where $R_{B}$ is

$$
\Sigma\left(B \otimes S(\Sigma(B))^{*}\right) \simeq \Sigma(B) \otimes \Sigma(S(\Sigma(B)))^{*} \stackrel{1 \otimes\left(\sigma_{\Sigma(B)}^{-1}\right)^{*}}{\longrightarrow} \Sigma(B) \otimes \Sigma(B)^{*} \stackrel{\eta_{\Sigma(B)}^{-1}}{\longrightarrow} I ;
$$

if $f: B \rightarrow C$ is an arrow in $\mathbb{B}$, we define $R(f)=f \otimes S\left(\Sigma\left(f^{-1}\right)\right)^{*}$. Now, to obtain a natural transformation $\nu$, let us consider an object $(N, n: \Sigma(N) \rightarrow I)$ in $\operatorname{Ker} \Sigma$. 
Up to the monoidal structure of $\Sigma$, the component of $\nu$ at the point $(B,(N, n))$ is given by the following composition

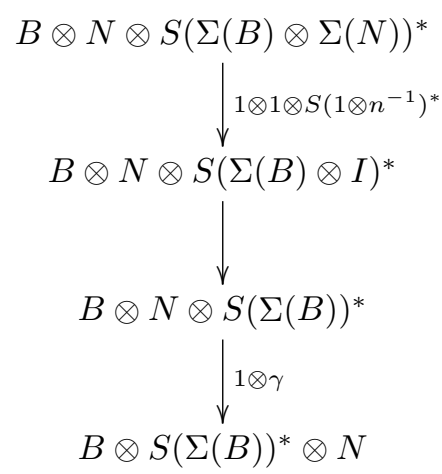

$2) \Rightarrow 1$ ) : Now we look for a functor $S:$ Coker $\Gamma \rightarrow \mathbb{B}$ (the natural transformation $\sigma: S \cdot P_{\Sigma} \Rightarrow 1_{\text {Coker } \Gamma}$ will be the obvious one). Let $X$ be an object in Coker $\Gamma$, we put $S(X)=X \otimes \Gamma(R(X))^{*}$. Consider now an arrow $[f, A]: X \rightarrow Y$ in Coker $\Gamma$, with $A$ in $\mathbb{A}$ and $f: X \rightarrow Y \otimes \Gamma(A)$ in $\mathbb{B}$. We define $S[f, A]$ by

$$
\begin{aligned}
& X \otimes \Gamma(R(X))^{*} \\
& \downarrow f \otimes \Gamma\left(R\left(f^{-1}\right)\right)^{*} \\
& Y \otimes \Gamma(A) \otimes \Gamma(R(Y \otimes \Gamma(A)))^{*} \\
& 1 \otimes 1 \otimes \Gamma\left(\nu_{Y, A}^{-1}\right)^{*} \\
& Y \Gamma(A) \otimes \Gamma(R(Y) \otimes A)^{*} \simeq Y \otimes \Gamma(R(Y))^{*}
\end{aligned}
$$

It is easy to check that $S$ is well defined; its functoriality follows from the coherence of $\nu$.

The lack of symmetry in Proposition 9.5 can be corrected. Indeed, since $\Sigma$ is monoidal, the natural transformation $\sigma$ can be equivalently replaced by a natural transformation $\Sigma(B \otimes S(C)) \rightarrow \Sigma(B) \otimes C$.

The difference between extensions and $\mathcal{F}$-extensions is stressed by the following fact.

Proposition 9.7. Let $(\Gamma, \varphi, \Sigma): \mathbb{A} \rightarrow \mathbb{B} \rightarrow \mathbb{C}$ be an $\mathcal{F}$-extension in SCG.

1) $\pi_{0} \mathbb{A} \stackrel{\pi_{0} \Gamma}{\longrightarrow} \pi_{0} \mathbb{B} \stackrel{\pi_{0} \Sigma}{\longrightarrow} \pi_{0} \mathbb{C}$ is an extension of abelian groups;

2) $\pi_{1} \mathbb{A} \stackrel{\pi_{1} \Gamma}{\longrightarrow} \pi_{1} \mathbb{B} \stackrel{\pi_{1} \Sigma}{\longrightarrow} \pi_{1} \mathbb{C}$ is a split extension of abelian groups.

Proof. Since $(\Gamma, \varphi, \Sigma)$ is an extension, in particular it is 2-exact. This implies the exactness of the two sequences of abelian groups (see Proposition 3.1 in [27]). Moreover, $\pi_{1} \Gamma$ is injective, because $\Gamma$ is faithful, and $\pi_{0} \Sigma$ is surjective, because $\Sigma$ is essentially surjective. By part 2 in Lemma $9.6, \pi_{0} \Gamma \cdot \pi_{0} \bar{R}=1_{\pi_{0} \mathbb{A}}$, so that $\pi_{0} \Gamma$ is injective and the $\pi_{0}$-sequence is an extension of abelian groups. As far as the $\pi_{1}$-sequence 
is concerned, we assume that the section $(S, \sigma)$ is normalized (Lemma 9.6). In this way, we can still define $\pi_{1} S: \pi_{1} \mathbb{C} \rightarrow \pi_{1} \mathbb{B}$ by

$$
I \stackrel{x}{\longrightarrow} I \quad \mapsto \quad I \stackrel{S_{I}}{\longrightarrow} S(I) \stackrel{S(x)}{\longrightarrow} S(I) \stackrel{S_{I}^{-1}}{\longrightarrow} I
$$

and it is a morphism of groups because $S$ is a functor. Finally, using once again the condition of Lemma 9.6, one checks that $\pi_{1} S \cdot \pi_{1} \Sigma=1_{\pi_{1} \mathbb{C}}$, so that the $\pi_{1^{-}}$sequence is a split extension of abelian groups.

Example 9.8. If, in Definition 9.4, we ask that the section $(S, \sigma)$ is only at a graph-theoretical level, we get another proper class strictly contained between $\mathcal{F}$ and EXT.

Example 9.9. An extension $(\Gamma, \varphi, \Sigma): \mathbb{A} \rightarrow \mathbb{B} \rightarrow \mathbb{C}$ of symmetric cat-groups is a $\pi_{1}$-extension if $\left(\pi_{1} \Gamma, \pi_{1} \Sigma\right): \pi_{1} \mathbb{A} \rightarrow \pi_{1} \mathbb{B} \rightarrow \pi_{1} \mathbb{C}$ is an extension of abelian groups. Equivalently, $(\Gamma, \varphi, \Sigma)$ is a $\pi_{1}$-extension when $\pi_{1} \Sigma$ is surjective. Since $\pi_{1}$ preserves pullbacks, $\pi_{1}$-extensions are a proper class.

Example 9.10. An extension $(\Gamma, \varphi, \Sigma): \mathbb{A} \rightarrow \mathbb{B} \rightarrow \mathbb{C}$ of symmetric cat-groups is a $\pi_{0}$-extension if $\left(\pi_{0} \Gamma, \pi_{0} \Sigma\right): \pi_{0} \mathbb{A} \rightarrow \pi_{0} \mathbb{B} \rightarrow \pi_{0} \mathbb{C}$ is an extension of abelian groups. Equivalently, $(\Gamma, \varphi, \Sigma)$ is a $\pi_{0}$-extension when $\pi_{0} \Gamma$ is injective. Since $\pi_{0}$ preserves pushouts, $\pi_{0}$-extensions are a proper class.

Example 9.11. From Propositions 5.1 and 5.2, we know that morphisms in SCG which are full functors are stable under pullbacks and pushouts. Therefore, one can wonder if, adding the conditions that $\Gamma$ and $\Sigma$ are full, one gets a proper class of extensions. This is not the case, because the trivial extension $\mathbb{A} \rightarrow \mathbb{A} \times \mathbb{C} \rightarrow \mathbb{C}$ is not of this kind, apart when $\mathbb{A}$ is equivalent to a one-object cat-group and $\mathbb{C}$ is equivalent to a discrete cat-group. There are no other possibilities. In fact, we have the following lemma.

Lemma 9.12. Let $(\Gamma, \varphi, \Sigma): \mathbb{A} \rightarrow \mathbb{B} \rightarrow \mathbb{C}$ be an extension.

1) $\Gamma$ is full if and only if $\pi_{1}(\mathbb{C})=0$;

2) $\Sigma$ is full if and only $\pi_{0}(\mathbb{A})=0$.

Proof. We prove part 1, part 2 is dual.

If: It follows from 1.3.8 in [16], because $\mathbb{C}$ is equivalent to $\operatorname{Coker} \Gamma$.

Only if: It follows from 1.3.2 in [16], because $\Gamma$ is equivalent to $e_{\Sigma}$.

Full extensions, that is extensions with $\Gamma$ and $\Sigma$ full, provide a quick classification of symmetric cat-groups in the following way. Let $A$ and $B$ be two abelian groups and call $\operatorname{Ep}(A, B)$ (Ep stays for "Epinglage", see [21]) the following 2-category:

- an object is a triple $(\mathbb{B}, a, c)$ with $\mathbb{B}$ in SCG and $a: \pi_{1}(\mathbb{B}) \rightarrow A, c: \pi_{0}(\mathbb{B}) \rightarrow C$ two isomorphisms of groups;

- a 1-cell $F:(\mathbb{B}, a, c) \rightarrow\left(\mathbb{B}^{\prime}, a^{\prime}, c^{\prime}\right)$ is a morphism $F: \mathbb{B} \rightarrow \mathbb{B}^{\prime}$ in SCG such that the 
following diagrams commute
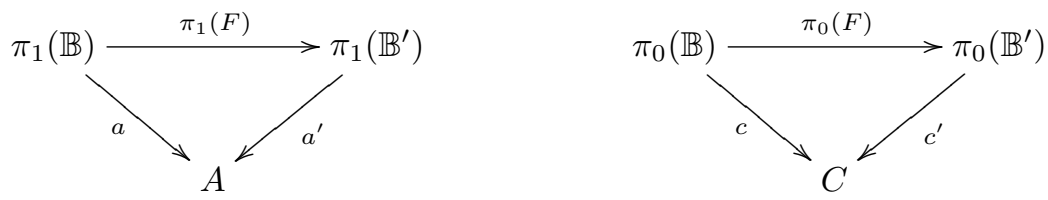

- a 2-cell $\lambda: F \Rightarrow F^{\prime}$ is simply a 2-cell in SCG.

Proposition 9.13. Let $A$ and $C$ be two abelian groups. The 2-categories $\operatorname{Ep}(A, C)$ and $\operatorname{EXT}(C[0], A[1])$ are biequivalent.

Proof. Given a symmetric cat-group $\mathbb{B}$, there is a canonical extension $\pi_{1}(\mathbb{B})[1] \rightarrow$ $\mathbb{B} \rightarrow \pi_{0}(\mathbb{B})[0]$. Conversely, if $(\Gamma, \varphi, \Sigma): A[1] \rightarrow \mathbb{B} \rightarrow C[0]$ is an extension, by Corollary 2.7 we have an exact sequence

$$
0 \longrightarrow A \stackrel{\pi_{1}(\Gamma)}{\longrightarrow} \pi_{1}(\mathbb{B}) \longrightarrow 0 \longrightarrow 0 \longrightarrow \pi_{0}(\mathbb{B}) \stackrel{\pi_{0}(\Sigma)}{\longrightarrow} C \longrightarrow 0
$$

so that $\pi_{1}(\Gamma)$ and $\pi_{0}(\Sigma)$ are isomorphisms. Finally, to check that these two constructions give a biequivalence, observe that in a 1-cell

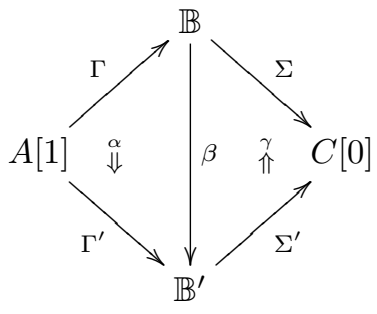

$\alpha$ is reduced to $\alpha_{I}$ which, by monoidality, is determined by $\Gamma, \beta$ and $\Gamma^{\prime}$; moreover, since $C[0]$ is discrete, $\gamma$ simply amount to say that $\pi_{0}(\beta) \cdot \pi_{0}\left(\Sigma^{\prime}\right)=\pi_{0}(\Sigma)$.

This classification does not seem very deep. Nevertheless, observe that a full extension $(\Gamma, \varphi, \Sigma): A[1] \rightarrow \mathbb{B} \rightarrow C[0]$ always has a functorial section for $\Sigma$, so that full extensions are a special case of $\mathcal{F}$-extensions. Therefore, we can apply to full extensions the cohomological classification of Section 15. Since the choice of a functorial section of $\Sigma$ amount to the choice of a set-theoretical section of $\pi_{0}(\Sigma)$ together with a clivage of $\mathbb{B}$, Theorem 15.2 and the previous proposition give the classification of symmetric cat-groups obtained by Sinh in $[\mathbf{2 1}]$. 


\section{The fundamental 2-exact sequences}

In this section we use the functors induced by the 2-functors of Proposition 6.4 on the classifying groupoids. We use the same notations :

$$
\begin{aligned}
& -\cdot E: \operatorname{Hom}(\mathbb{D}, \mathbb{C}) \longrightarrow \operatorname{Ext}(\mathbb{D}, \mathbb{A}) \\
& G \cdot-: \operatorname{Ext}(\mathbb{C}, \mathbb{A}) \longrightarrow \operatorname{Ext}(\mathbb{D}, \mathbb{A}) \\
& E \cdot-: \operatorname{Hom}(\mathbb{A}, \mathbb{D}) \longrightarrow \operatorname{Ext}(\mathbb{C}, \mathbb{D}) \\
& -\cdot F: \operatorname{Ext}(\mathbb{C}, \mathbb{A}) \longrightarrow \operatorname{Ext}(\mathbb{C}, \mathbb{D}) .
\end{aligned}
$$

All these groupoids are pointed groupoids, and the various functors are functors of pointed groupoids (in fact, they are all in SCG, but the structure of symmetric cat-group of $\operatorname{Ext}(\mathbb{C}, \mathbb{A})$ will be discussed later $)$ : the point in $\operatorname{Hom}(\mathbb{D}, \mathbb{C})$ is the zero functor $0: \mathbb{D} \rightarrow \mathbb{C}$, the point in $\operatorname{Ext}(\mathbb{C}, \mathbb{A})$ is the trivial extension $T(\mathbb{A}, \mathbb{C})$. We say that a diagram in the 2-category $\mathrm{Grpd}^{*}$ of pointed groupoids

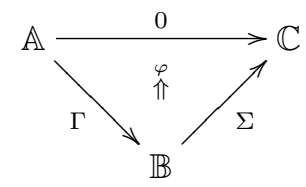

is a 2-exact sequence if the comparison functor $\Gamma_{0}: \mathbb{A} \rightarrow \operatorname{Ker} \Sigma$ is full and essentially surjective.

The next lemma is a step towards Proposition 10.2. It is an obvious exercise on the left exactness of representable 2 -functors.

Lemma 10.1. Consider a symmetric cat-group $\mathbb{D}$, a 1-cell $\Sigma: \mathbb{B} \rightarrow \mathbb{C}$ in $\mathrm{SCG}$ and its kernel

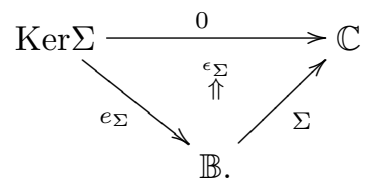

In the next diagram, the comparison functor $K$ is an equivalence

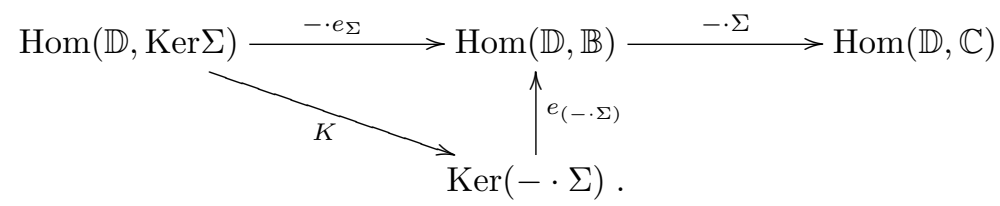


Proposition 10.2. Let $E=(\Gamma, \varphi, \Sigma)$

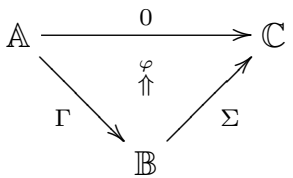

be an extension in SCG and fix a symmetric cat-group $\mathbb{D}$. The sequence in Grpd*

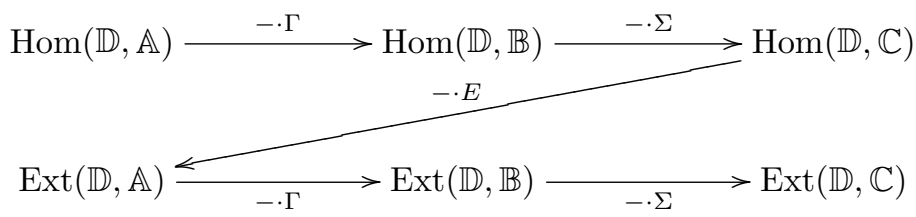

(equipped with the pointed natural transformations specified in the proof) is 2-exact at each point.

If $\mathcal{P}$ is a proper class of extensions and if $E \in \mathcal{P}(\mathbb{C}, \mathbb{A})$, the same result holds replacing $\mathrm{EXT}$ by $\mathcal{P}$.

Proof. The 2-exactness of

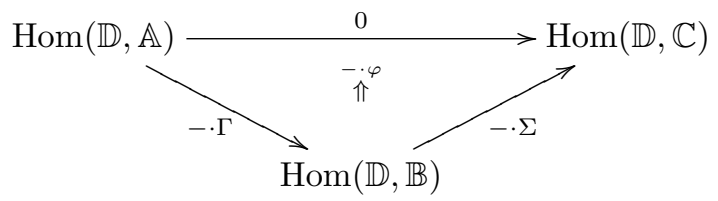

is attested by Lemma 10.1. We give now a detailed proof of the 2-exactness in $\operatorname{Ext}(\mathbb{D}, \mathbb{A})$. The argument for the 2-exactness in $\operatorname{Hom}(\mathbb{D}, \mathbb{C})$ and $\operatorname{Ext}(\mathbb{D}, \mathbb{B})$ is similar and we give only the description of the pointed natural transformations involved. We use Lemma 4.5 and its consequence discussed at the end of Section 4, as wall as the dual argument.

2-exactness in $\operatorname{Ext}(\mathbb{D}, \mathbb{A})$ : let $G: \mathbb{D} \rightarrow \mathbb{C}$ be in $\operatorname{Hom}(\mathbb{D}, \mathbb{C})$ and consider the pullback along $\Sigma$ and the pushout along $\Gamma$, i.e. the extension $(G \cdot E) \cdot \Gamma$, as in the following diagrams
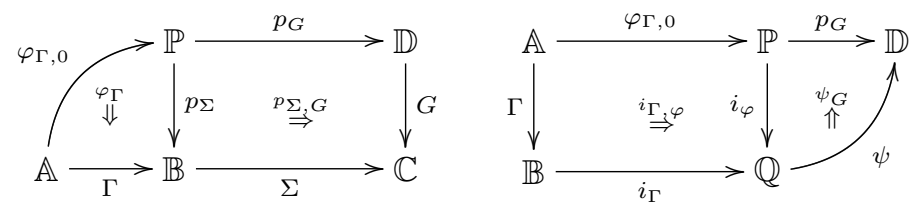

with $\varphi_{0}: \varphi_{\Gamma, 0} \cdot p_{G} \Rightarrow 0$ and $\psi_{0}: i_{\Gamma} \cdot \psi \Rightarrow 0$. Using $\varphi_{\Gamma}$, we get a retraction in SCG in 
the following way

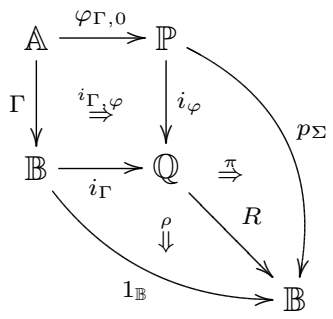

This gives the component at $G$ of a pointed natural transformation $\xi:(-\cdot E) \cdot(-$. $\Gamma) \Rightarrow 0$.

Consider now the factorization through the kernel of $(-\cdot \Gamma)$ induced by the previous natural transformation

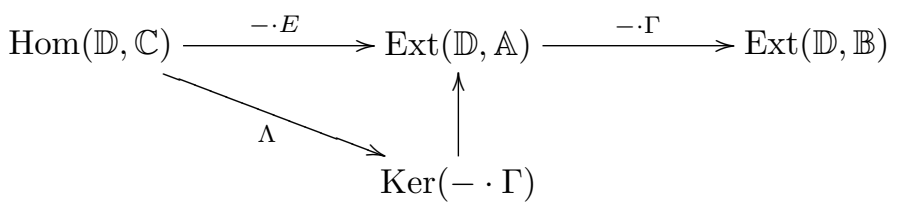

1) $\Lambda$ is essentially surjective on objects : consider an extension $(L, \mu, M): \mathbb{A} \rightarrow \mathbb{E} \rightarrow$ $\mathbb{D}$, its pushout along $\Gamma$

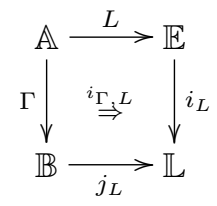

and the factorization

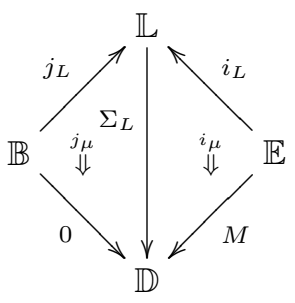

making commutative the following diagram

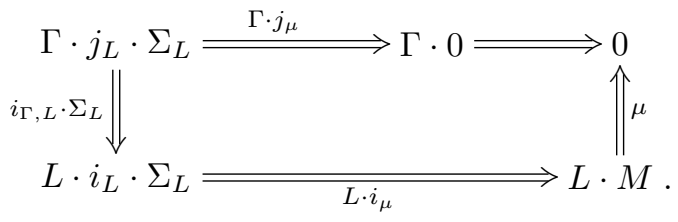


Assume that there is a retraction in SCG

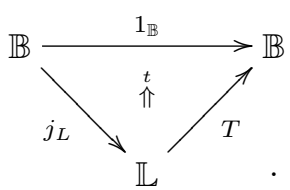

Using this retraction, we can construct a 2-cell in SCG

$$
\tau: L \cdot i_{L} \cdot T \cdot \Sigma \stackrel{i_{\Gamma, L}^{-1} \cdot T \cdot \Sigma}{=} \Gamma \cdot j_{L} \cdot T \cdot \Sigma \stackrel{\Gamma \cdot t \cdot \Sigma}{\Longrightarrow} \Gamma \cdot \Sigma \stackrel{\varphi}{\Longrightarrow} 0 .
$$

Since $(M, \mu)$ is a cokernel of $L$, we get $G: \mathbb{D} \rightarrow \mathbb{C}$ in $\operatorname{Hom}(\mathbb{D}, \mathbb{C})$ and a 2-cell $\tau^{\prime}: M \cdot G \Rightarrow i_{L} \cdot T \cdot \Sigma$ in SCG such that the following diagram commutes

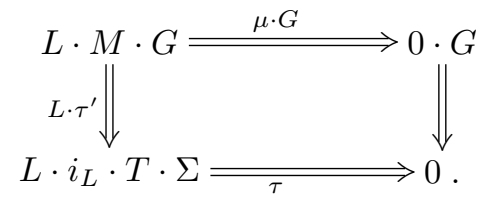

Consider now the extension $G \cdot E$ as at the beginning of the proof. We look for a 1-cell $(\alpha, \beta, \gamma):(L, \mu, M) \rightarrow G \cdot E$ in $\operatorname{EXT}(\mathbb{D}, \mathbb{A})$. The universal property of the pullback $\mathbb{P}$ gives a factorization

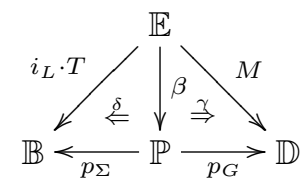

such that

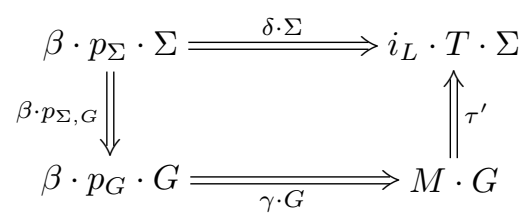

commutes. We can construct two 2-cells in SCG

$$
\begin{aligned}
& \alpha_{G}: L \cdot \beta \cdot p_{G} \stackrel{L \cdot \gamma}{\Longrightarrow} L \cdot M \stackrel{\mu}{\Longrightarrow} 0 \stackrel{\varphi_{0}^{-1}}{\Longrightarrow} \varphi_{\Gamma, 0} \cdot p_{G} \\
& \alpha_{\Sigma}: L \cdot \beta \cdot p_{\Sigma} \stackrel{L \cdot \delta}{\Longrightarrow} L \cdot i_{L} \cdot T \stackrel{i_{\Gamma, L}^{-1} \cdot T}{\Longrightarrow} \Gamma \cdot j_{L} \cdot T \stackrel{\Gamma \cdot t}{\Longrightarrow} \Gamma \stackrel{\varphi_{\Gamma}^{-1}}{\Longrightarrow} \varphi_{\Gamma, 0} \cdot p_{\Sigma} .
\end{aligned}
$$

Since $\alpha_{G}$ and $\alpha_{\Sigma}$ are compatible with $p_{\Sigma, G}$, the universal property of the pullback $\mathbb{P}$ gives a unique 2-cell $\alpha: L \cdot \beta \Rightarrow \varphi_{\Gamma, 0}$ in SCG such that $\alpha \cdot p_{G}=\alpha_{G}$ and $\alpha \cdot p_{\Sigma}=\alpha_{\Sigma}$. In particular, the equation $\alpha \cdot p_{G}=\alpha_{G}$ express the condition on $(\alpha, \beta, \gamma)$ to be a 1-cell $(L, \mu, M) \rightarrow G \cdot E$ in $\operatorname{EXT}(\mathbb{D}, \mathbb{A})$. It remains to prove that $(\alpha, \beta, \gamma)$ is a morphism in the kernel of $(-\cdot \Gamma)$. For this, observe that the universal property of the pushout $\mathbb{L}$ gives a factorization $q: \mathbb{L} \rightarrow \mathbb{Q}, m: i_{L} \cdot q \Rightarrow \beta \cdot i_{\varphi}, n: j_{L} \cdot q \Rightarrow i_{\Gamma}$ 
such that

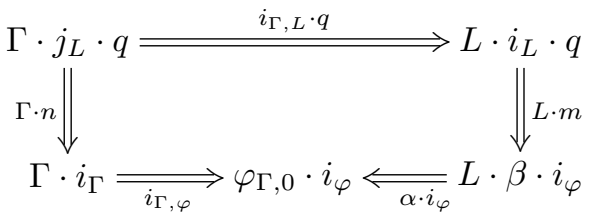

commutes. Now we have two retractions of $j_{L}:(T, t)$ and the one given by

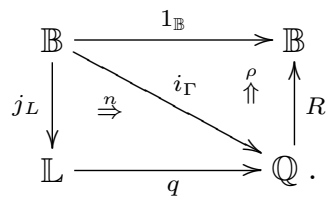

We have also a 2-cell in SCG

$$
i_{L} \cdot q \cdot R=\beta \cdot i_{\varphi} \cdot R=\stackrel{\beta \cdot \pi}{\Longrightarrow} \beta \cdot p_{\Sigma} \stackrel{\delta}{\Longrightarrow} i_{L} \cdot T
$$

and, since the following diagram commutes

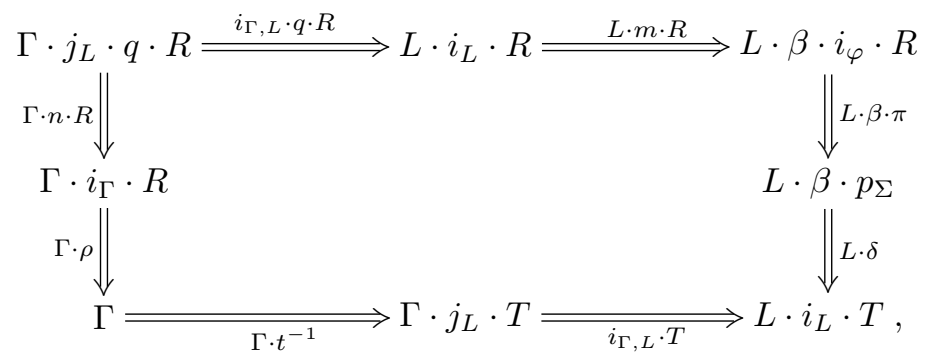

the universal property of the pushout $\mathbb{L}$ gives a unique 2 -cell in SCG, $\lambda: T \Rightarrow q \cdot R$, such that
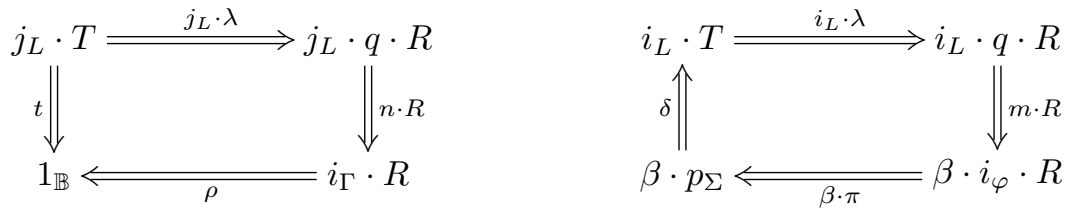

commute. In particular, the first equation exactly means that $\lambda$ commutes with the retraction.

2) $\Lambda$ is full : consider $G, \bar{G}$ in $\operatorname{Hom}(\mathbb{D}, \mathbb{C})$, the extensions $G \cdot E$ and $\bar{G} \cdot E$ and a 1-cell $(\alpha, \beta, \gamma): G \cdot E \rightarrow \bar{G} \cdot E$ in $\operatorname{EXT}(\mathbb{D}, \mathbb{A})$. Consider also the retractions in SCG
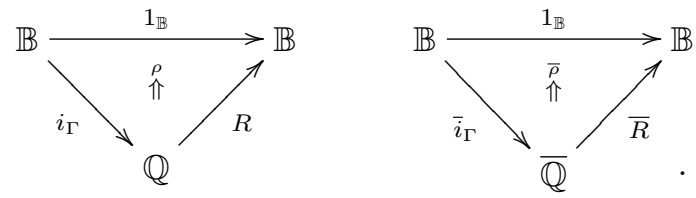
The universal property of the pushout $\mathbb{Q}$ gives a factorization $q, a, b$ as in the following diagram

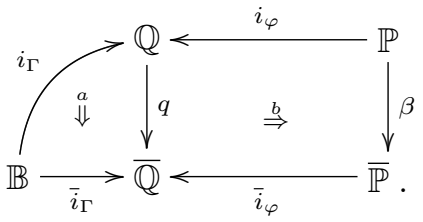

In this way we have a second retraction for $i_{\Gamma}$ :

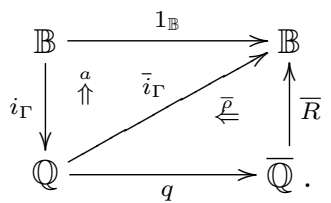

Assume that there is a 2-cell $\lambda: R \Rightarrow q \cdot \bar{R}$ in SCG making commutative the following diagram

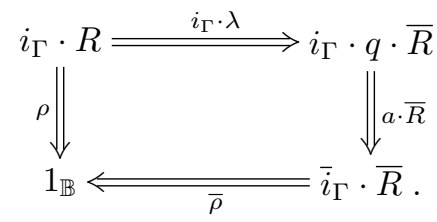

We can build up the following 2-cell

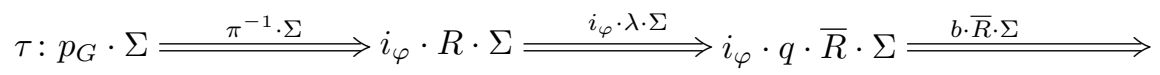

$\beta \cdot \bar{i}_{\varphi} \cdot \bar{R} \cdot \Sigma \stackrel{\beta \cdot \bar{\pi} \cdot \Sigma}{\Longrightarrow} \beta \cdot \bar{p}_{\Sigma} \cdot \Sigma \stackrel{\beta \cdot p_{\Sigma, \bar{G}}}{\Longrightarrow} \beta \cdot p_{\bar{G}} \cdot \bar{G} \stackrel{\gamma \cdot \bar{G}}{\Longrightarrow} p_{G} \cdot \bar{G}$.

Since the diagram

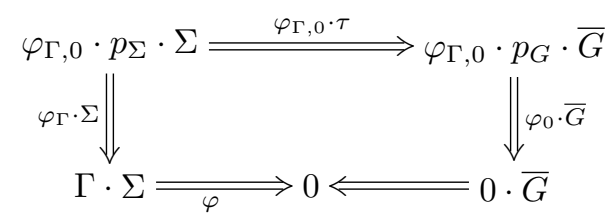

commutes, the universal property of $\left(p_{G}, \varphi_{0}\right)$ (it is the cokernel of $\left.\varphi_{\Gamma, 0}\right)$ gives a unique 2-cell $\mu: G \Rightarrow \bar{G}$ in SCG making commutative the following diagram

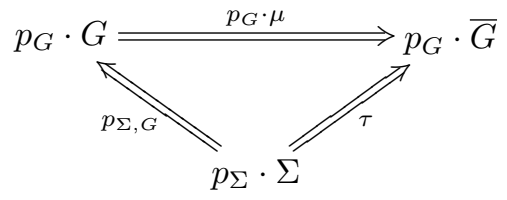


It remains to prove that $(\alpha, \beta, \gamma)$ and $\mu \cdot E$ coincide as arrows in $\operatorname{Ext}(\mathbb{D}, \mathbb{A})$. The functor part of $\mu \cdot E$ is given by the following factorization through the pullback $\overline{\mathbb{P}}$

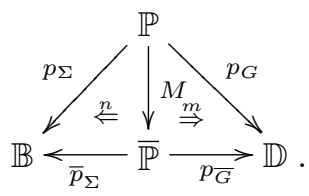

We need a 2-cell $x: M \Rightarrow \beta$ in $\operatorname{EXT}(\mathbb{D}, \mathbb{A})$ and, for this, we can consider

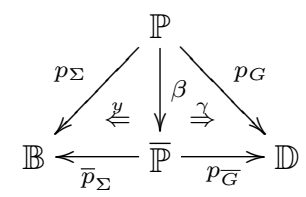

where $y$ is given by the following composition

$$
\beta \cdot \bar{p}_{\Sigma} \stackrel{\beta \cdot \bar{\pi}^{-1}}{\Longrightarrow} \beta \cdot \bar{i}_{\varphi} \cdot \bar{R} \stackrel{b^{-1} \cdot \bar{R}}{\Longrightarrow} i_{\varphi} \cdot q \cdot \bar{R} \stackrel{i_{\varphi} \cdot \lambda^{-1}}{\Longrightarrow} i_{\varphi} \cdot R \stackrel{\pi}{\Longrightarrow} p_{\Sigma}
$$

Since the following diagram commutes

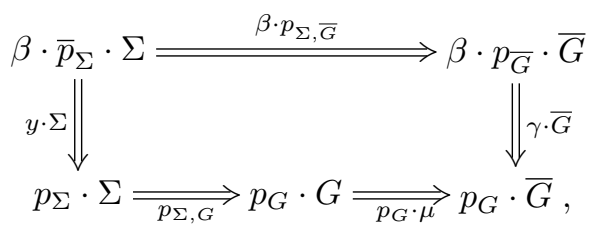

the universal property of the pullback $\overline{\mathbb{P}}$ gives a unique 2-cell $x: M \Rightarrow \beta$ in SCG such that
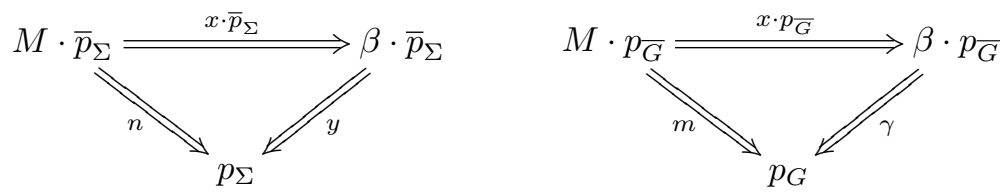

commute. The verification that $x$ satisfies also the first condition of 2-cell in $\operatorname{EXT}(\mathbb{D}, \mathbb{A})$ needs a full description of $\mu \cdot E$ (see Lemma 6.2) and we omit it.

2-exactness in $\operatorname{Hom}(\mathbb{D}, \mathbb{C})$ : let $F: \mathbb{D} \rightarrow \mathbb{B}$ be in $\operatorname{Hom}(\mathbb{D}, \mathbb{B})$ and consider the following pullback

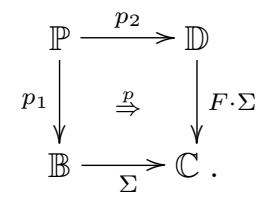


We get a section of $p_{2}$ in SCG in the following way

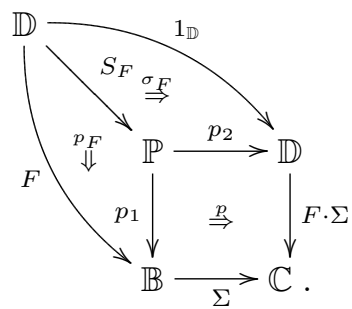

Via Lemma 4.5, the section $\left(S_{F}, \sigma_{F}\right)$ gives the component at $F$ of a pointed natural transformation $\zeta:(-\cdot \Sigma) \cdot(-\cdot E) \Rightarrow 0$.

2-exactness in $\operatorname{Ext}(\mathbb{D}, \mathbb{B}):$ let $L=(F, \psi, G): \mathbb{A} \rightarrow \mathbb{E} \rightarrow \mathbb{D}$ be an extension of $\mathbb{A}$ by $\mathbb{D}$. Consider the following pushouts and factorizations
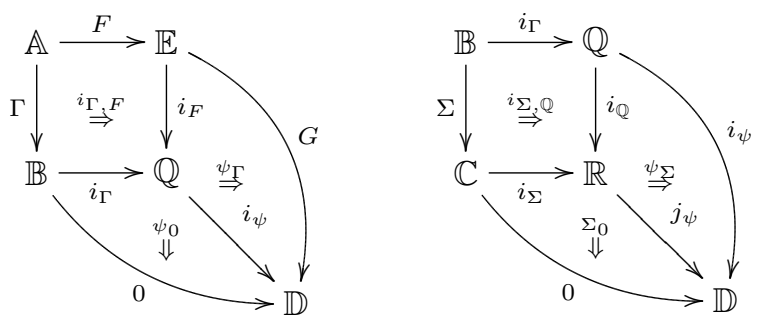

which gives $(L \cdot \Gamma) \cdot \Sigma$. We get a factorization

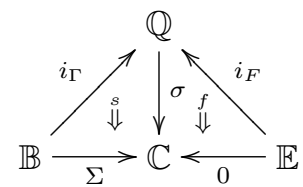

and, finally, a factorization

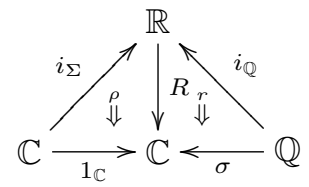

making commutative the following diagram

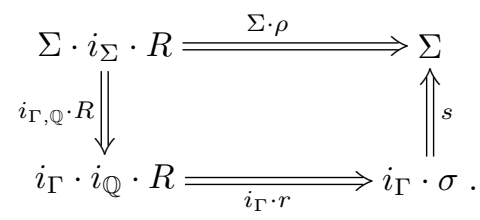

In particular, via the dual of Lemma 4.5, the retraction $(R, \rho)$ of $i_{\Sigma}$ gives the component at the point $L$ of a pointed natural transformation $\chi:(-\cdot \Gamma) \cdot(-\cdot \Sigma) \Rightarrow 0$.

Let us write explicitly the duals of Lemma 10.1 and Proposition 10.2. 
Lemma 10.3. Consider a symmetric cat-group $\mathbb{D}$, a 1 -cell $\Gamma: \mathbb{A} \rightarrow \mathbb{B}$ in SCG and its cokernel

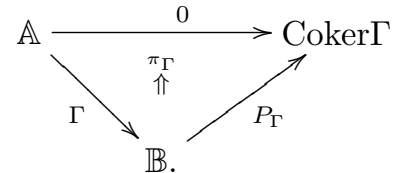

In the next diagram, the comparison functor $K$ is an equivalence

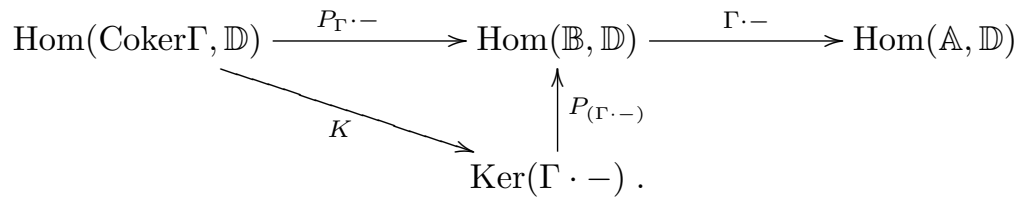

Proposition 10.4. Let $E=(\Gamma, \varphi, \Sigma)$

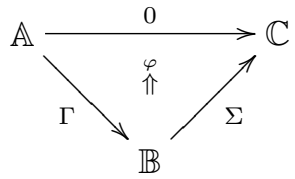

be an extension in SCG and fix a symmetric cat-group $\mathbb{D}$. The sequence in $\mathrm{Grpd}^{*}$

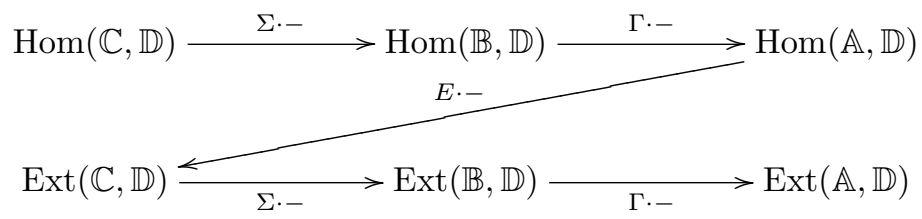

(equipped with four specific pointed natural transformations) is 2-exact at each point. If $\mathcal{P}$ is a proper class of extensions and if $E \in \mathcal{P}(\mathbb{C}, \mathbb{A})$, the same result holds replacing $\mathrm{EXT}$ by $\mathcal{P}$.

\section{Projective objects}

In this section we consider projective objects in SCG. Definitions and properties for injective objects are dual and are left to the reader. We fix a proper class $\mathcal{P}$ of extensions. The notion of $\mathcal{P}$-projective object corresponding to our definition of extension is the following one.

Definition 11.1. Let $\mathbb{A}$ be a symmetric cat-group ; $\mathbb{A}$ is $\mathcal{P}$-projective if, for any $\mathcal{P}$-epi $F: \mathbb{B} \rightarrow \mathbb{A}$, there exists a section in SCG (i.e. a 1-cell $S: \mathbb{A} \rightarrow \mathbb{B}$ and a 2-cell $\sigma: S \cdot F \Rightarrow 1_{\mathbb{A}}$ in $\left.\mathrm{SCG}\right)$.

Using pullbacks in SCG (see Section 5), the previous definition can be restated in the following way : a symmetric cat-group $\mathbb{A}$ is $\mathcal{P}$-projective if, for each 1-cell $G: \mathbb{A} \rightarrow \mathbb{C}$ in $\mathrm{SCG}$ and for each $\mathcal{P}$-epi $F: \mathbb{B} \rightarrow \mathbb{C}$, there exist $G^{\prime}: \mathbb{A} \rightarrow \mathbb{B}$ and $g: G^{\prime} \cdot F \Rightarrow G$ in SCG. 
Corollary 11.2. Let $\mathbb{A}$ be in $\mathrm{SCG}$; the following conditions are equivalent :

1) $\mathbb{A}$ is $\mathcal{P}$-projective ;

2) For each $\mathbb{B}$ in $\mathrm{SCG}$, any $\mathcal{P}$-extension of $\mathbb{B}$ by $\mathbb{A}$ splits ;

3) For each $\mathbb{B}$ in $\mathrm{SCG}$, the classifying groupoid cl $\mathcal{P}(\mathbb{A}, \mathbb{B})$ of $\mathcal{P}(\mathbb{A}, \mathbb{B})$ is connected.

In the next corollary we use that the sequence of Proposition 10.4 (as well as that of Proposition 10.2) is a 2-exact sequence of symmetric cat-groups, and not only of pointed groupoids (the cat-group structure of $c l \mathcal{P}$ is discussed in Section 13). By Proposition 6.2 in [16], we can then translate its 2 -exactness saying that the factorizations through the appropriate cokernels are full and faithful functors.

Corollary 11.3. Consider an extension $E=(\Gamma, \varphi, \Sigma): \mathbb{A} \rightarrow \mathbb{B} \rightarrow \mathbb{C}$ of $\mathbb{A}$ by $\mathbb{C}$. Assume that $\mathbb{B}$ is a $\mathcal{P}$-projective object in $\mathrm{SCG}$ and that $E \in \mathcal{P}(\mathbb{C}, \mathbb{A})$. Then, for any symmetric cat-group $\mathbb{D}, c l \mathcal{P}(\mathbb{C}, \mathbb{D})$ is equivalent in $\mathrm{SCG}$ to the cokernel of

$$
\Gamma \cdot-: \operatorname{Hom}(\mathbb{B}, \mathbb{D}) \rightarrow \operatorname{Hom}(\mathbb{A}, \mathbb{D}) .
$$

Proof. By Proposition 10.4, we have a 2-exact sequence

$$
\operatorname{Hom}(\mathbb{A}, \mathbb{D}) \stackrel{E \cdot-}{\longrightarrow} \operatorname{cl} \mathcal{P}(\mathbb{C}, \mathbb{D}) \stackrel{\Sigma \cdot-}{\longrightarrow} \operatorname{cl} \mathcal{P}(\mathbb{B}, \mathbb{D})
$$

and then its image by $\pi_{0}: \mathrm{SCG} \rightarrow \mathrm{Ab}$ is an exact sequence of abelian groups. Moreover, by Corollary $11.2, \pi_{0}(\operatorname{cl} \mathcal{P}(\mathbb{B}, \mathbb{D}))=0$, so that $\pi_{0}(E \cdot-)$ is surjective. Equivalently, $E \cdot-$ is essentially surjective on objects. Consider now the factorization $\epsilon$ of $E \cdot-$ through the cokernel of $\Gamma \cdot-$

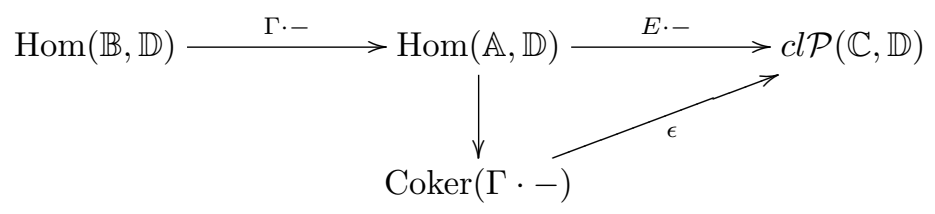

By 2-exactness in $\operatorname{Hom}(\mathbb{A}, \mathbb{D})$ of the sequence in Proposition 10.4, $\epsilon$ is full and faithful. It is also essentially surjective, because $E \cdot-$ is essentially surjective.

\section{Exactness of Hom}

In this section we show that EXT measures the non exactness of representable 2 -functors. We consider the case of covariant representable 2-functors and we leave to the reader the controvariant case.

Lemma 12.1. Let

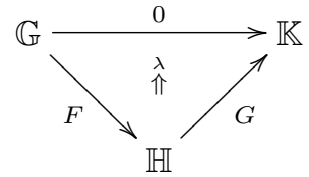

be a 2-exact sequence in SCG. The functor $F$ is essentially surjective if and only if $\pi_{0}(G)=0$. 
Proof. Only if : Since $\pi_{0}(F)$ is surjective, $\operatorname{Coker} \pi_{0}(F)=0$. But Coker $\pi_{0}(F) \simeq$ $\pi_{0}(\operatorname{Coker} F)$, and $\pi_{0}(G)$ factors through $\pi_{0}(\operatorname{Coker} F)$.

If : Since $\left(\pi_{0}(F), \pi_{0}(G)\right): \pi_{0}(\mathbb{G}) \rightarrow \pi_{0}(\mathbb{H}) \rightarrow \pi_{0}(\mathbb{K})$ is exact and $\pi_{0}(G)=0$, then $\pi_{0}(F)$ is surjective, that is $F$ is essentially surjective.

Proposition 12.2. Let $E=(\Gamma, \varphi, \Sigma): \mathbb{A} \rightarrow \mathbb{B} \rightarrow \mathbb{C}$ be an extension. Consider a symmetric cat-group $\mathbb{D}$ and the morphism $-\cdot E: \operatorname{Hom}(\mathbb{D}, \mathbb{C}) \rightarrow \operatorname{Ext}(\mathbb{D}, \mathbb{A})$. The sequence $(-\cdot \Gamma,-\cdot \varphi,-\cdot \Sigma): \operatorname{Hom}(\mathbb{D}, \mathbb{A}) \rightarrow \operatorname{Hom}(\mathbb{D}, \mathbb{B}) \rightarrow \operatorname{Hom}(\mathbb{D}, \mathbb{C})$ is an extension if and only if $\pi_{0}(-\cdot E)=0$.

Proof. By Lemma 10.1, we know that $-\cdot \Gamma$ is equivalent to the kernel of $-\cdot \Sigma$. Hence, the result follows applying the previous lemma to the sequence

$$
\operatorname{Hom}(\mathbb{D}, \mathbb{B}) \stackrel{-\cdot \Sigma}{\longrightarrow} \operatorname{Hom}(\mathbb{D}, \mathbb{C}) \stackrel{-\cdot E}{\longrightarrow} \operatorname{Ext}(\mathbb{D}, \mathbb{A})
$$

which is 2-exact by Proposition 10.2.

Corollary 12.3. Let $\mathbb{D}$ be a symmetric cat-group. The following conditions are equivalent :

1) $\mathbb{D}$ is projective (i.e. $\mathcal{P}$-projective for $\mathcal{P}=\mathrm{EXT}$ );

2) For any essentially surjective morphism $\Sigma: \mathbb{B} \rightarrow \mathbb{C}$, the functor

$$
-\cdot \Sigma: \operatorname{Hom}(\mathbb{D}, \mathbb{B}) \rightarrow \operatorname{Hom}(\mathbb{D}, \mathbb{C})
$$

is essentially surjective ;

3) For any extension $(\Gamma, \varphi, \Sigma): \mathbb{A} \rightarrow \mathbb{B} \rightarrow \mathbb{C}$, the sequence

$$
(-\cdot \Gamma,-\cdot \varphi,-\cdot \Sigma): \operatorname{Hom}(\mathbb{D}, \mathbb{A}) \rightarrow \operatorname{Hom}(\mathbb{D}, \mathbb{B}) \rightarrow \operatorname{Hom}(\mathbb{D}, \mathbb{C})
$$

is an extension.

Proof. The non-trivial implication is 1) $\Rightarrow 2$ ), which follows from Proposition 12.2 and Corollary 11.2.

We consider now $\mathcal{F}$-extensions (Example 9.3). In this case, Proposition 12.2 has a slightly different (and a little bit more difficult to prove) formulation.

Proposition 12.4. Consider an $\mathcal{F}$-extension $E=(\Gamma, \varphi, \Sigma): \mathbb{A} \rightarrow \mathbb{B} \rightarrow \mathbb{C}$ in $\mathrm{SCG}$ and a symmetric cat-group $\mathbb{D}$. The diagram $(\mathbb{D}, E)$ :

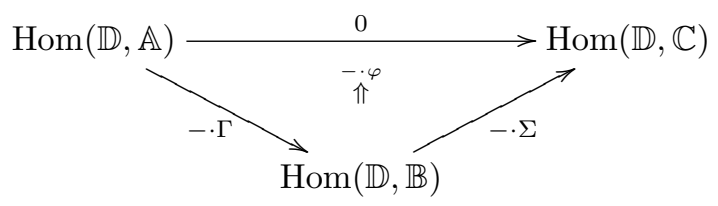

is an $\mathcal{F}$-extension if and only if the 2-functor

$$
-\cdot E: \operatorname{Hom}(\mathbb{D}, \mathbb{C}) \rightarrow \mathcal{F}(\mathbb{D}, \mathbb{A})
$$

is isomorphic to the 2-functor which sends each 1-cell $G: \mathbb{D} \rightarrow \mathbb{C}$ into the trivial extension $T(\mathbb{A}, \mathbb{D})$ and each 2-cell $\mu: G \Rightarrow G^{\prime}$ into the identity of $T(\mathbb{A}, \mathbb{D})$. 
Proof. By Lemma 10.1, we have to deal only with a functorial section for $-\cdot \Sigma$. We use the notations of Lemma 6.1 and Lemma 6.2.

$(\Leftarrow)$ Assume that we have a 2-natural transformation

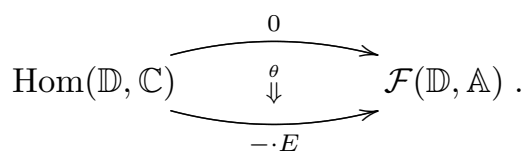

This means that, for each $G: \mathbb{D} \rightarrow \mathbb{C}$ in $\operatorname{Hom}(\mathbb{D}, \mathbb{C})$, we have a 1-cell $\left(\alpha_{G}, \theta_{G}, \gamma_{G}\right): T(\mathbb{A}, \mathbb{D}) \rightarrow G \cdot E$ in $\mathcal{F}(\mathbb{D}, \mathbb{A})$

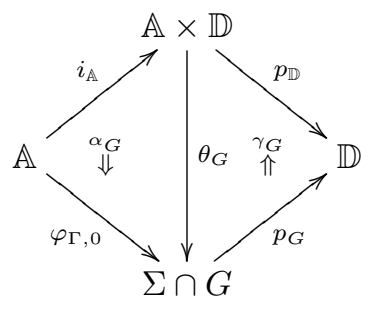

and, for each $\mu: G \Rightarrow G^{\prime}$ in $\operatorname{Hom}(\mathbb{D}, \mathbb{C})$, we have a 2 -cell $\theta_{\mu}:\left(\alpha_{G}, \theta_{G}, \gamma_{G}\right) \cdot(\mu \cdot E) \Rightarrow$ $\left(\alpha_{G^{\prime}}, \theta_{G^{\prime}}, \gamma_{G^{\prime}}\right)$. Now we can define $T: \operatorname{Hom}(\mathbb{D}, \mathbb{C}) \rightarrow \operatorname{Hom}(\mathbb{D}, \mathbb{B})$ by

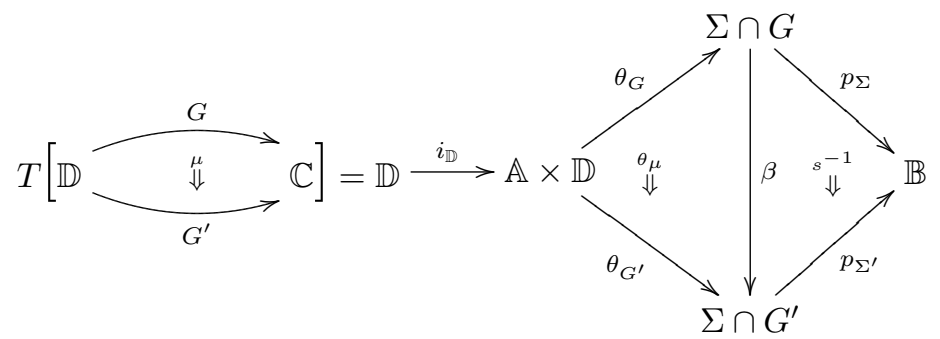

and it is functorial by 2-naturality of $\theta$.

We need a natural transformation

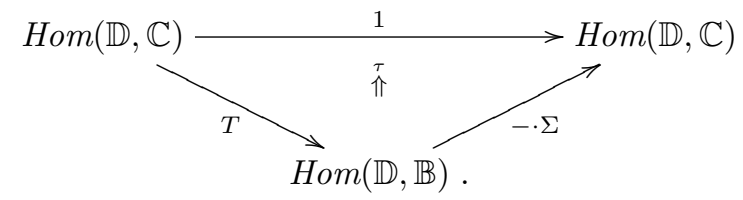

For each $G \in \operatorname{Hom}(\mathbb{D}, \mathbb{C})$, we define $\tau(G): T(G) \cdot \Sigma \Rightarrow G$ by the following diagram

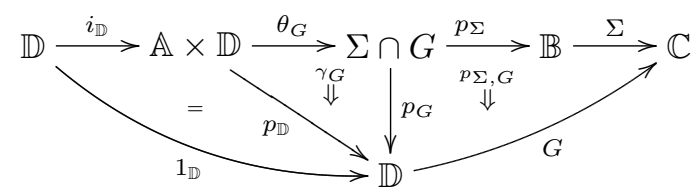

The naturality of $\tau$ comes from the compatibility of $\beta$ with $p_{\Sigma, G}$ and $p_{\Sigma, G^{\prime}}$ and from the fact that $\theta_{\mu}$ is a 2-cell in $\mathcal{F}(\mathbb{D}, \mathbb{A})$. 
$(\Rightarrow)$ Assume that we have a functorial section

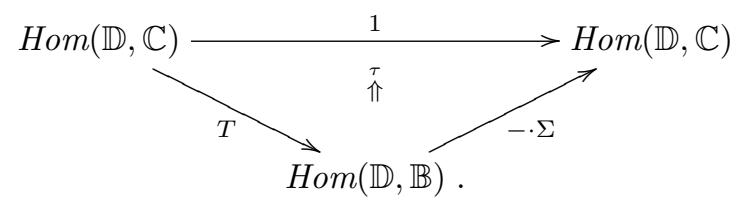

and consider, for $G$ in $\operatorname{Hom}(\mathbb{D}, \mathbb{C})$, the 2-cell $\tau(G): T(G) \cdot \Sigma \Rightarrow G$ in SCG. By the universal property of the pullback $\Sigma \cap G$, we obtain $g, g_{1}, g_{2}$ in SCG as in the following diagram

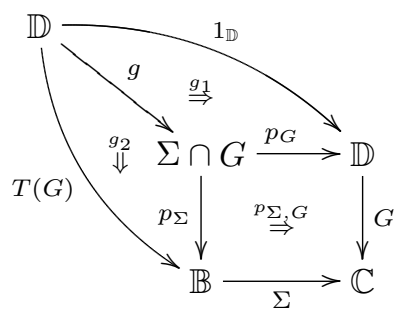

making commutative the following diagram

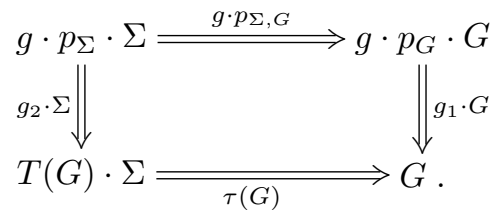

In particular, $\left(g, g_{1}\right)$ is an object of $\operatorname{Split}\left(p_{G}\right)$. By Lemma $4.5,\left(g, g_{1}\right)$ corresponds to a 1-cell $\left(\alpha_{G}, \theta_{G}, \gamma_{G}\right): T(\mathbb{A}, \mathbb{D}) \rightarrow G \cdot E$ in $\mathcal{F}(\mathbb{D}, \mathbb{A})$.

Now, let $\mu: G \Rightarrow G^{\prime}$ be in $\operatorname{Hom}(\mathbb{D}, \mathbb{C})$. We need a 2 -cell in $\mathcal{F}(\mathbb{D}, \mathbb{A})$ as in the following diagram

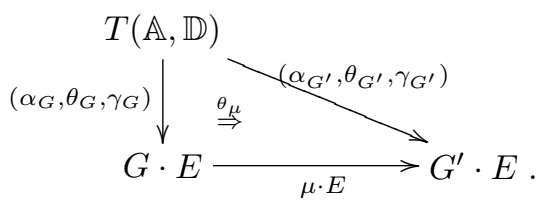

By Lemma 4.5, we can equivalently look for an arrow

$$
\lambda_{\mu}:\left(g \cdot \beta, g \cdot \beta \cdot p_{G^{\prime}} \stackrel{g \cdot \gamma}{=} g \cdot p_{G} \stackrel{g_{1}}{\Longrightarrow} 1_{\mathbb{D}}\right) \Rightarrow\left(g^{\prime}, g^{\prime} \cdot p_{G^{\prime}}^{\stackrel{g_{1}^{\prime}}{\Longrightarrow}} 1_{\mathbb{D}}\right)
$$


in $\operatorname{Split}\left(p_{G^{\prime}}\right)$, where $g^{\prime}, g_{1}^{\prime}$ and $g_{2}^{\prime}$ are as in the following diagram

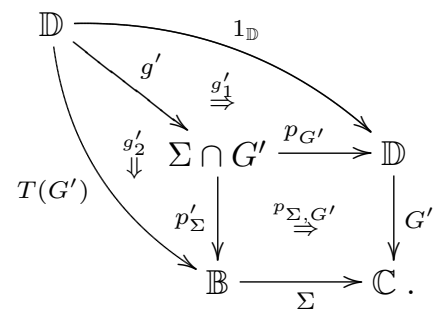

Consider the 2-cells

$$
\begin{aligned}
& \lambda_{\mathbb{D}}: g \cdot \beta \cdot p_{G^{\prime}} \stackrel{g \cdot \gamma}{\Longrightarrow} g \cdot p_{G} \stackrel{g_{1}}{\Longrightarrow} 1_{\mathbb{D}} \stackrel{\left(g_{1}^{\prime}\right)^{-1}}{\Longrightarrow} g^{\prime} \cdot p_{G^{\prime}} \\
& \lambda_{\mathbb{B}}: g \cdot \beta \cdot p_{\Sigma}^{\prime} \stackrel{g \cdot s}{\Longrightarrow} g \cdot p_{\Sigma} \stackrel{g_{2}}{\Longrightarrow} T(G) \stackrel{T(\mu)}{\Longrightarrow} T\left(G^{\prime} \stackrel{\left(g_{2}^{\prime}\right)^{-1}}{\Longrightarrow} g^{\prime} \cdot p_{\Sigma}^{\prime} \cdot\right.
\end{aligned}
$$

Using the three equations satisfied by $\beta, \gamma, s, g, g_{1}, g_{2}, g^{\prime}, g_{1}^{\prime}, g_{2}^{\prime}$ and the naturality of $\tau$, one can check that the following diagram commutes

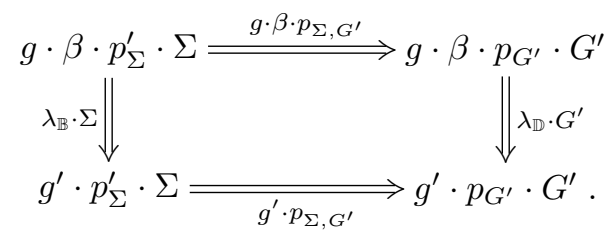

The universal property of the pullback $\Sigma \cap G^{\prime}$ gives then a unique 2-cell $\lambda_{\mu}: g \cdot \beta \Rightarrow g^{\prime}$ such that $\lambda_{\mu} \cdot p_{G^{\prime}}=\lambda_{\mathbb{D}}$ and $\lambda_{\mu} \cdot p_{\Sigma}^{\prime}=\lambda_{\mathbb{B}}$. The first of these equations precisely means that $\lambda_{\mu}$ is an arrow in $\operatorname{Split}\left(p_{G^{\prime}}\right)$.

\section{Baer sum}

In this section, we introduce Baer sum for extensions of symmetric cat-groups. The construction closely follows the 1-dimensional analogue and we don't give the proof. If $A, C$ are two abelian groups, the group of extensions of $A$ by $C$ is in fact the group of connected components of the category of extensions of $A$ by $C$, which is a symmetric cat-group. In the same way, the structure of symmetric cat-group on $\operatorname{Ext}(\mathbb{C}, \mathbb{A})$, for $\mathbb{A}$ and $\mathbb{C}$ two symmetric cat-groups, could be extended to a monoidal structure on the 2-groupoid $\operatorname{EXT}(\mathbb{C}, \mathbb{A})$. We limit our attention to the groupoid $\operatorname{Ext}(\mathbb{C}, \mathbb{A})$ only for sake of simplicity.

Lemma 13.1. With the notations of Definition 8.1.

1) Consider an extension $E=(\Gamma, \varphi, \Sigma)$, a 1-cell $G: \mathbb{D} \rightarrow \mathbb{C}$ in SCG and the 
following 1-cell $G \cdot E \rightarrow E$ (see Lemma 6.1)

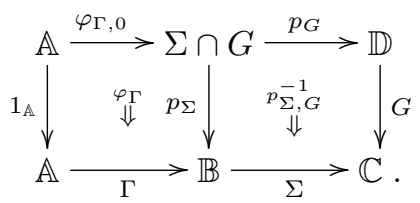

For any 1 -cell $(F, \alpha, \beta, \gamma, G): E^{\prime} \rightarrow E$

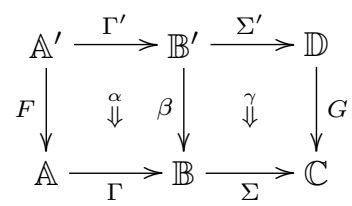

there is a 1-cell $\left(F^{\prime}, \alpha^{\prime}, \beta^{\prime}, \gamma^{\prime}, G^{\prime}\right): E^{\prime} \rightarrow G \cdot E$ and a 2-cell

$$
(x, y, z):\left(F^{\prime}, \alpha^{\prime}, \beta^{\prime}, \gamma^{\prime}, G^{\prime}\right) \cdot\left(1_{\mathbb{A}}, \varphi_{\Gamma}, p_{\Sigma}, p_{\Sigma, G}^{-1}, G\right) \Rightarrow(F, \alpha, \beta, \gamma, G) .
$$

Moreover, for any other 1-cell $\left(F^{\prime \prime}, \alpha^{\prime \prime}, \beta^{\prime \prime}, \gamma^{\prime \prime}, G^{\prime \prime}\right): E^{\prime} \rightarrow G \cdot E$ with a 2-cell

$$
(u, v, w):\left(F^{\prime \prime}, \alpha^{\prime \prime}, \beta^{\prime \prime}, \gamma^{\prime \prime}, G^{\prime \prime}\right) \cdot\left(1_{\mathbb{A}}, \varphi_{\Gamma}, p_{\Sigma}, p_{\Sigma, G}^{-1}, G\right) \Rightarrow(F, \alpha, \beta, \gamma, G),
$$

there is a unique 2-cell $(a, b, c):\left(F^{\prime}, \alpha^{\prime}, \beta^{\prime}, \gamma^{\prime}, G^{\prime}\right) \Rightarrow\left(F^{\prime \prime}, \alpha^{\prime \prime}, \beta^{\prime \prime}, \gamma^{\prime \prime}, G^{\prime \prime}\right)$ making commutative the following diagram

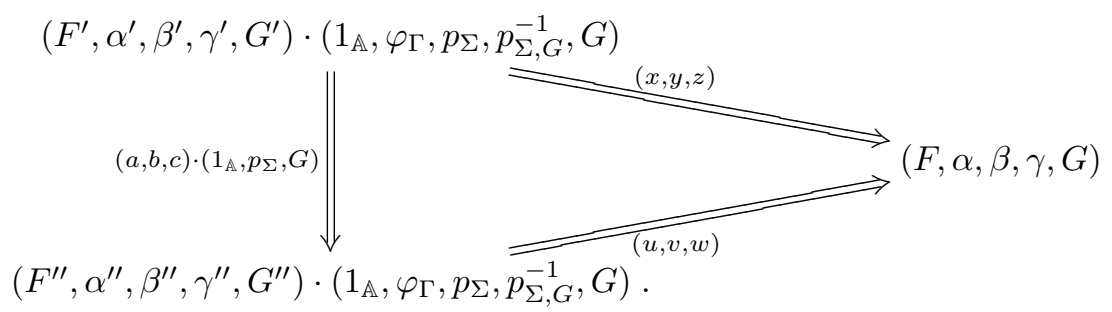

2) An analogue universal property holds if one considers an extension $E=(\Gamma, \varphi, \Sigma)$, a 1-cell $F: \mathbb{A} \rightarrow \mathbb{D}$ in SCG and the 1-cell $E \rightarrow E \cdot F$ as in Lemma 6.3

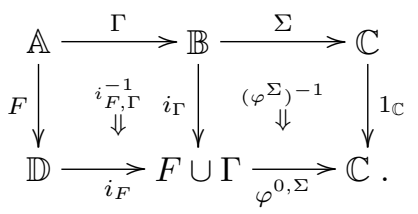

Corollary 13.2. Consider an extension $E=(\Gamma, \varphi, \Sigma): \mathbb{A} \rightarrow \mathbb{B} \rightarrow \mathbb{C}$ and four 1-cells in SCG

$$
\mathbb{A} \stackrel{F}{\longrightarrow} \mathbb{D} \stackrel{H}{\longrightarrow} \mathbb{F} \quad \mathbb{G} \stackrel{K}{\longrightarrow} \mathbb{E} \stackrel{G}{\longrightarrow} \mathbb{C} .
$$

The following pairs of functors are naturally isomorphic in a canonical way :

1) $\operatorname{Ext}(\mathbb{C}, \mathbb{A}) \stackrel{-\cdot F}{\longrightarrow} \operatorname{Ext}(\mathbb{C}, \mathbb{D}) \stackrel{-\cdot H}{\longrightarrow} \operatorname{Ext}(\mathbb{C}, \mathbb{F})$

and $\operatorname{Ext}(\mathbb{C}, \mathbb{A}) \longrightarrow \operatorname{Ext}(\mathbb{C}, \mathbb{F})$; 
2) $\operatorname{Ext}(\mathbb{C}, \mathbb{A}) \stackrel{G \cdot-}{\longrightarrow} \operatorname{Ext}(\mathbb{E}, \mathbb{A}) \stackrel{K \cdot-}{\longrightarrow} \operatorname{Ext}(\mathbb{G}, \mathbb{A})$

and $\operatorname{Ext}(\mathbb{C}, \mathbb{A}) \longrightarrow(K \cdot G) \cdot-\quad \operatorname{Ext}(\mathbb{G}, \mathbb{A}) ;$

3) $\operatorname{Ext}(\mathbb{C}, \mathbb{A}) \stackrel{-\cdot F}{\longrightarrow} \operatorname{Ext}(\mathbb{C}, \mathbb{D}) \stackrel{G \cdot-}{\longrightarrow} \operatorname{Ext}(\mathbb{E}, \mathbb{D})$

and $\operatorname{Ext}(\mathbb{C}, \mathbb{A}) \stackrel{G \cdot-}{\longrightarrow} \operatorname{Ext}(\mathbb{E}, \mathbb{A}) \stackrel{-\cdot F}{\longrightarrow} \operatorname{Ext}(\mathbb{E}, \mathbb{D}) ;$

4) $\operatorname{Hom}(\mathbb{A}, \mathbb{D}) \stackrel{E \cdot-}{\longrightarrow} \operatorname{Ext}(\mathbb{C}, \mathbb{D}) \stackrel{G \cdot-}{\longrightarrow} \operatorname{Ext}(\mathbb{E}, \mathbb{D})$

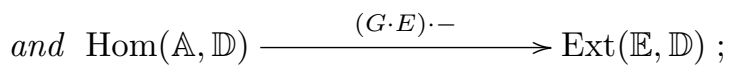

5) $\operatorname{Hom}(\mathbb{E}, \mathbb{C}) \stackrel{-\cdot E}{\longrightarrow} \operatorname{Ext}(\mathbb{E}, \mathbb{A}) \stackrel{-\cdot F}{\longrightarrow} \operatorname{Ext}(\mathbb{E}, \mathbb{D})$

and $\operatorname{Hom}(\mathbb{E}, \mathbb{C}) \longrightarrow \operatorname{Ext}(\mathbb{E}, \mathbb{D})$.

We can now describe the monoidal structure of the groupoid $\operatorname{Ext}(\mathbb{C}, \mathbb{A})$. Given two extensions
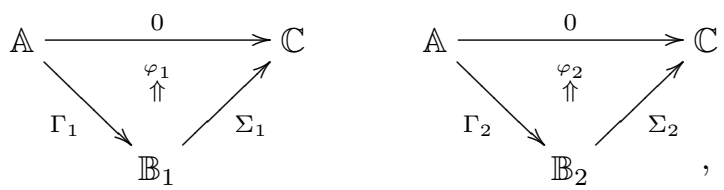

we get a new extension considering

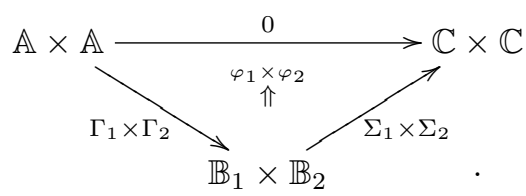

This construction extends to a 2-functor

$$
\oplus: \operatorname{EXT}(\mathbb{C}, \mathbb{A}) \times \operatorname{EXT}(\mathbb{C}, \mathbb{A}) \rightarrow \operatorname{EXT}(\mathbb{C} \times \mathbb{C}, \mathbb{A} \times \mathbb{A})
$$

Consider now the diagonal and codiagonal 1-cells in SCG

$$
\Delta: \mathbb{C} \rightarrow \mathbb{C} \times \mathbb{C} \quad \nabla: \mathbb{C} \times \mathbb{C} \rightarrow \mathbb{C} .
$$

Using part 3 of Corollary 13.2, we can define the tensor product in $\operatorname{Ext}(\mathbb{C}, \mathbb{A})$ in the following way

$$
\otimes: \operatorname{Ext}(\mathbb{C}, \mathbb{A}) \times \operatorname{Ext}(\mathbb{C}, \mathbb{A}) \stackrel{\oplus}{\longrightarrow} \operatorname{Ext}(\mathbb{C} \times \mathbb{C}, \mathbb{A} \times \mathbb{A}) \stackrel{\Delta \cdot-\cdot \nabla}{\longrightarrow} \operatorname{Ext}(\mathbb{C}, \mathbb{A}) .
$$

The unit object is given by the trivial extension $T(\mathbb{A}, \mathbb{C})$ and the inverse of an extension $E$ is $E \cdot\left(1_{\mathbb{A}}^{*}\right)$, where $1_{\mathbb{A}}^{*}: \mathbb{A} \rightarrow \mathbb{A}$ is the 1-cell in SCG defined by $X \stackrel{f}{\longrightarrow} Y \sim X^{*} \stackrel{\left(f^{-1}\right)^{*}}{\longrightarrow} Y^{*}$. It is a (very) long but essentially straightforward work to complete this definition with associativity, commutativity and unit isomorphisms, and to check the axioms for a symmetric cat-group. For example, 
the associativity isomorphism is given by the canonical natural isomorphisms in the following diagram (where we write $(\mathbb{C}, \mathbb{A})$ instead of $\operatorname{Ext}(\mathbb{C}, \mathbb{A}))$ :

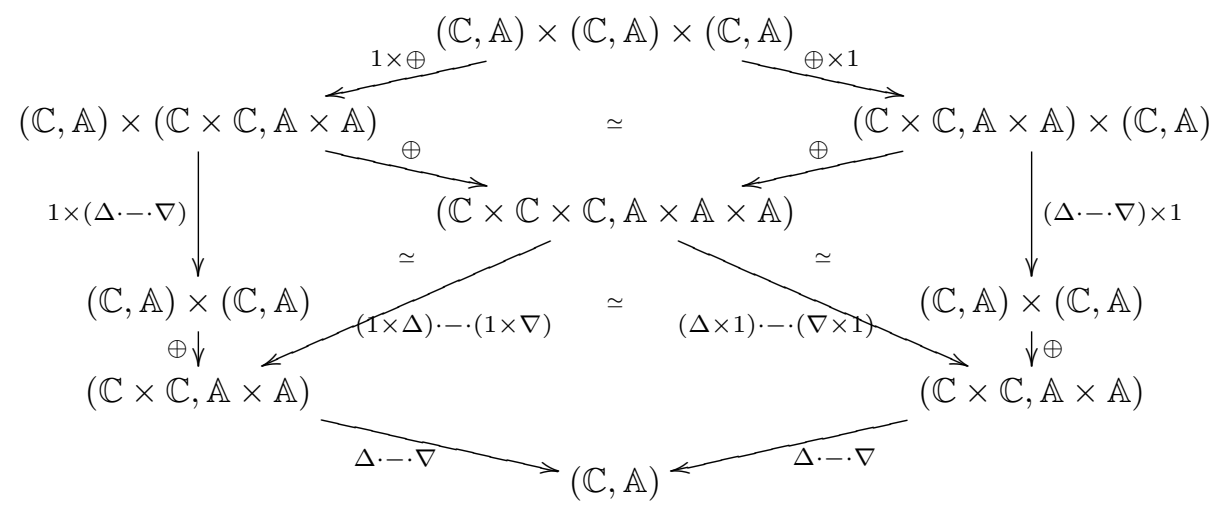

Remark 13.3. Observe that the functors listed at the beginning of Section 10 are now 1-cells in SCG. In particular, the sequences of Propositions 10.2 and 10.4 are 2-exact sequences in SCG.

Remark 13.4. The results of this section hold if we replace EXT by a proper class $\mathcal{P}$ of extensions, provided that the 2 -functor $\oplus: \operatorname{EXT}(\mathbb{C}, \mathbb{A}) \times \operatorname{EXT}(\mathbb{C}, \mathbb{A}) \rightarrow$ $\operatorname{EXT}(\mathbb{C} \times \mathbb{C}, \mathbb{A} \times \mathbb{A})$ restricts to $\mathcal{P}$. This is the case for all the examples given in Section 9 .

\section{Cobords and cocycles}

In [20], extensions of (non necessarily symmetric) cat-groups are classified. A catgroup $\mathbb{A}$ is a bicategory with only one object, and we can consider the bicategory $\operatorname{Bieq}(\mathbb{A})$ of biequivalences from $\mathbb{A}$ to $\mathbb{A}$. Clearly, $\operatorname{Bieq}(\mathbb{A})$ is a monoidal bicategory under composition. Given another cat-group $\mathbb{C}$, we can look at it as a monoidal bicategory with only identity 2-arrows. The main result in $[\mathbf{2 0}]$ is a biequivalence $\operatorname{EXT}(\mathbb{C}, \mathbb{A}) \simeq \operatorname{Bimon}(\mathbb{C}, \operatorname{Bieq}(\mathbb{A}))$, where an object in $\operatorname{Bimon}(\mathbb{C}, \operatorname{Bieq}(\mathbb{A}))$ is a morphism of bicategories $\mathbb{C} \rightarrow \operatorname{Bieq}(\mathbb{A})$ which takes into account the monoidal structures in a sense made precise in $[\mathbf{2 0}]$. (The referee pointed out to us that $\mathcal{F}$-extensions (Definition 9.4) correspond to those objects of $\operatorname{Bimon}(\mathbb{C}, \operatorname{Bieq}(\mathbb{A}))$ such that the underlying homomorphism of bicategories is equivalent to the null homomorphism.)

In this section and in the next one, we look for a cohomological classification of extensions more on the line of Schreier theory. Having in mind factor sets, the class of extensions which seems appropriate for such a theory is the class of $\mathcal{F}$-extensions. Once again, for the reasons explained in the introduction, we restrict our attention to symmetric cat-groups.

The first step towards a cohomological classification of $\mathcal{F}$-extensions is to find an appropriate notion of cocycle. For this, consider $\mathbb{A}$ and $\mathbb{C}$ in SCG and let us look 
for a monoidal structure on $\mathbb{A} \times \mathbb{C}$ such that

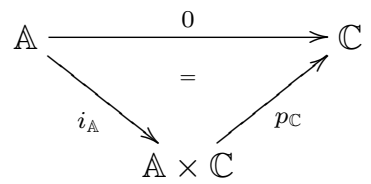

is an extension (necessarily, an $\mathcal{F}$-extension).

Let us call for a moment $*: \mathbb{A} \times \mathbb{C} \times \mathbb{A} \times \mathbb{C} \rightarrow \mathbb{A} \times \mathbb{C}$ the new tensor product in $\mathbb{A} \times \mathbb{C}$. If we assume that

$$
(A, X) *(B, Y)=((A, I) *(B, I)) \otimes((I, X) *(I, Y))
$$

(where $\otimes$ is the point-wise tensor product in $\mathbb{A} \times \mathbb{C}$ ), we get that $(A, X) *(B, Y)$ must be equal to $(A \otimes B \otimes F(X, Y), X \otimes Y)$, for $F: \mathbb{C} \times \mathbb{C} \rightarrow \mathbb{A}$ any functor. Moreover, the associativity, unit and symmetry isomorphisms for $(\mathbb{A} \times \mathbb{C}, *)$ give rise to four natural isomorphisms (compare with 5.2.1.11, 5.2.1.14 and 5.2.1.15 in [20])

$$
\begin{aligned}
& \text { - } c_{F}(X, Y, Z): F(X, Y) \otimes F(X \otimes Y, Z) \rightarrow F(Y, Z) \otimes F(X, Y \otimes Z) ; \\
& \text { - } r_{F}(X): F(X, I) \rightarrow I \\
& \text { - } l_{F}(Y): F(I, Y) \rightarrow I \\
& \text { - } s_{F}(X, Y): F(X, Y) \rightarrow F(Y, X) .
\end{aligned}
$$

Finally, the four coherence axioms for the isomorphisms in $(\mathbb{A} \times \mathbb{C}, *)$ are equivalent to the following four axioms on $c_{F}, r_{F}, l_{F}$ and $s_{F}$, expressed in terms of commutative diagrams (we omit the associativity isomorphism in $\mathbb{A}$ ) :

(C1) The two following compositions coincide

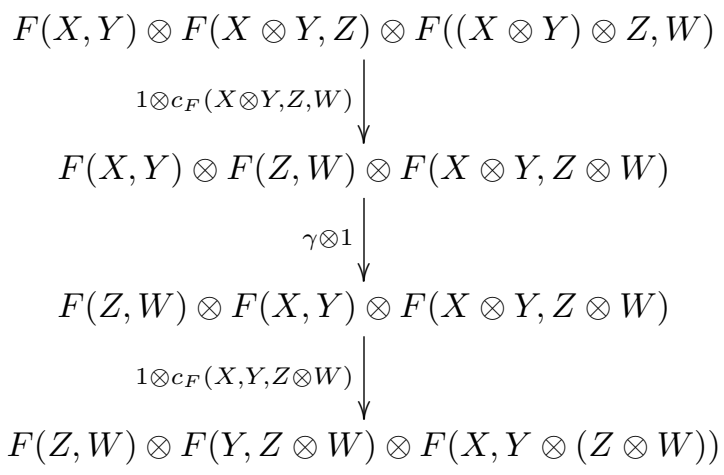




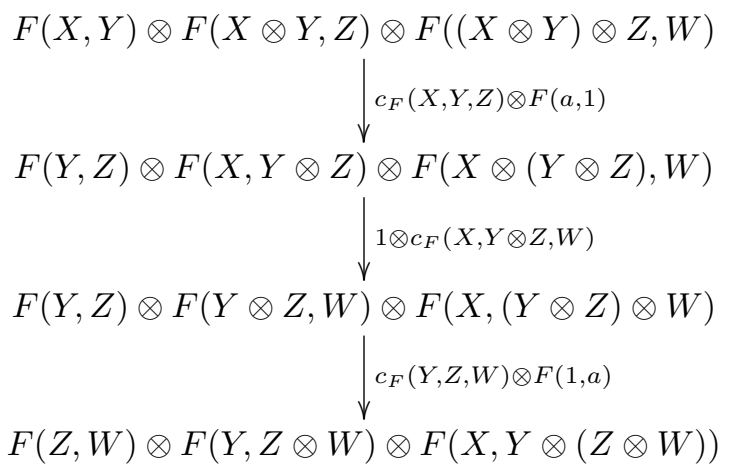

$(\mathrm{C} 2)$

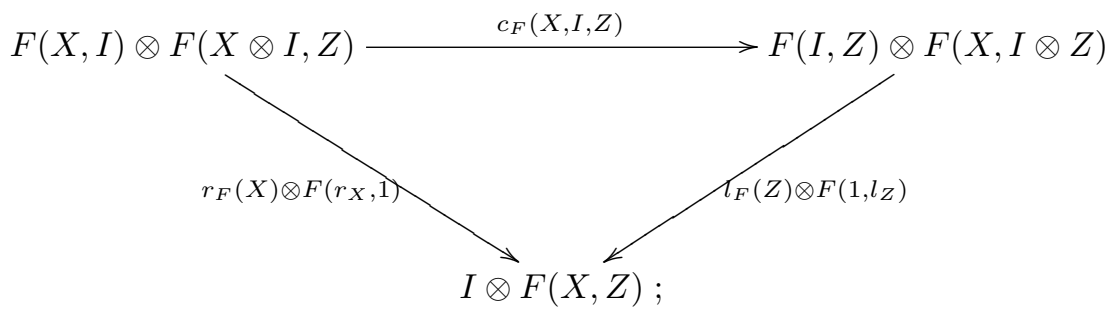

(C3)

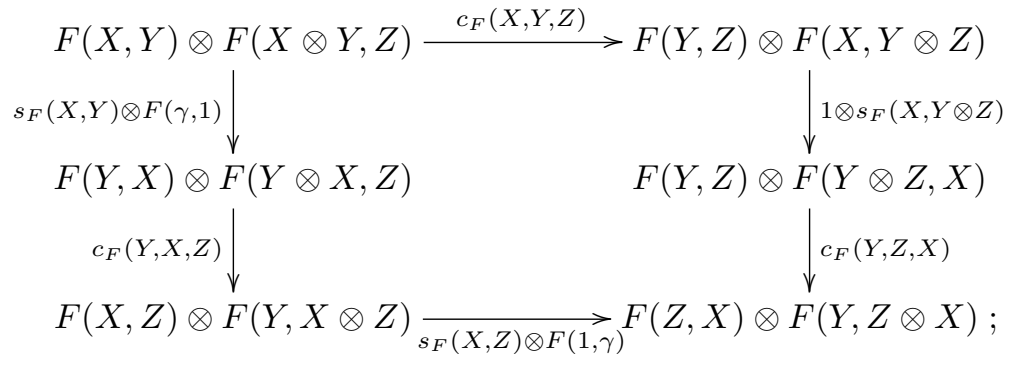

(C4)

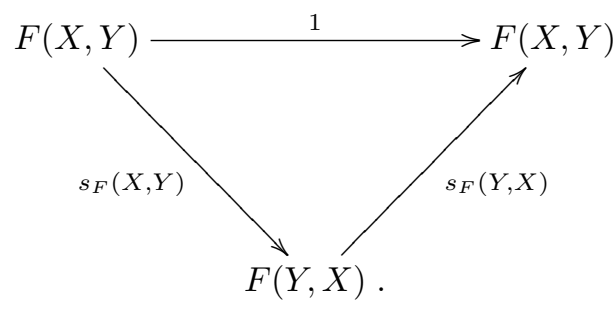

Definition 14.1. Let $\mathbb{A}, \mathbb{C}$ be two symmetric cat-groups.

1) A symmetric 2-cocycle of $\mathbb{C}$ with coefficients in $\mathbb{A}$ (a cocycle, for short) is given by $F=\left(F, c_{F}, r_{F}, l_{F}, s_{F}\right)$ where $F: \mathbb{C} \times \mathbb{C} \rightarrow \mathbb{A}$ is a functor and 
- $c_{F}(X, Y, Z): F(X, Y) \otimes F(X \otimes Y, Z) \rightarrow F(Y, Z) \otimes F(X, Y \otimes Z)$,

- $r_{F}(X): F(X, I) \rightarrow I$,

- $l_{F}(Y): F(I, Y) \rightarrow I$,

- $s_{F}(X, Y): F(X, Y) \rightarrow F(Y, X)$

are natural isomorphisms satisfying the axioms (C1),(C2), (C3) and (C4).

2) Given two cocycles $F$ and $F^{\prime}$, a morphism of cocycles is a natural transformation $\alpha: F \Rightarrow F^{\prime}$ making commutative the following diagrams (MC1)

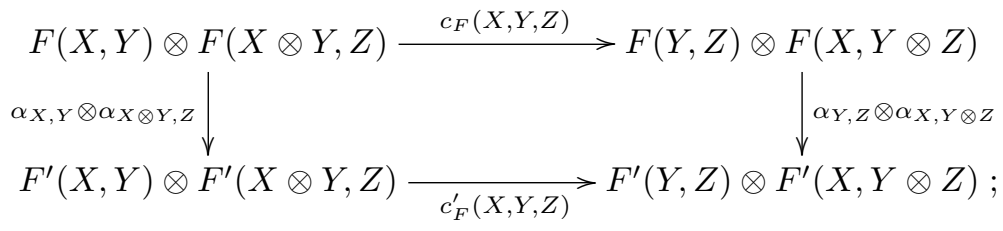

$(M C 2)$
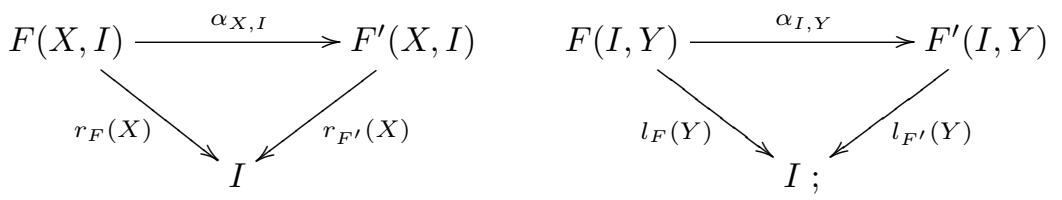

$(M C 3)$

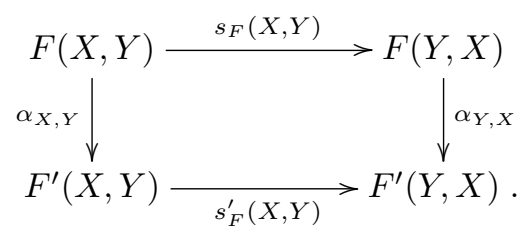

3) A normalized 2-cobord of $\mathbb{C}$ with coefficients in $\mathbb{A}$ (a cobord, for short) is given by $G=\left(G, G_{I}\right)$, where $G: \mathbb{C} \rightarrow \mathbb{A}$ is a functor and $G_{I}: I \rightarrow G I$ is an arrow in $\mathbb{A}$.

4) Given two cobords $G$ and $G^{\prime}$, a morphism of cobords is a natural transformation $\alpha: G \Rightarrow G^{\prime}$ making commutative the following diagram

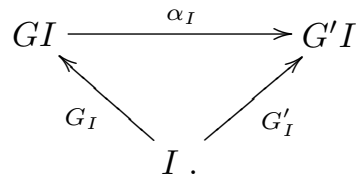

Proposition 14.2. Let $\mathbb{A}, \mathbb{C}$ be in $\mathrm{SCG}$.

1) The data of the previous definition give a category $\mathcal{Z}^{2}(\mathbb{C}, \mathbb{A})$ of cocycles and a category $\mathcal{B}^{2}(\mathbb{C}, \mathbb{A})$ of cobords ;

2) $\mathcal{Z}^{2}(\mathbb{C}, \mathbb{A})$ and $\mathcal{B}^{2}(\mathbb{C}, \mathbb{A})$ have a structure of symmetric cat-groups induced pointwise by that of $\mathbb{A}$. 
If $G \in \mathcal{B}^{2}(\mathbb{C}, \mathbb{A})$, we can construct a functor $\delta G: \mathbb{C} \times \mathbb{C} \rightarrow \mathbb{A}$ in the following way

- $(\delta G)(X, Y)=G(X \otimes Y) \otimes G(Y)^{*} \otimes G(X)^{*}$

- if $f: X \rightarrow X^{\prime}$ and $g: Y \rightarrow Y^{\prime}$ are in $\mathbb{C}$, then $(\delta G)(f, g)=G(f \otimes g) \otimes G\left(g^{-1}\right)^{*} \otimes$ $G\left(f^{-1}\right)^{*}$.

Moreover (using $G_{I}$, the symmetry of $\mathbb{C}$ and $\mathbb{A}$ and, for $c_{\delta G}$, unit and counit of $G(X \otimes Y) \dashv G(X \otimes Y)^{*}$ and $\left.G(Y \otimes Z) \dashv G(Y \otimes Z)^{*}\right)$ one can construct four natural isomorphisms $c_{\delta G}, r_{\delta G}, l_{\delta G}, s_{\delta G}$ so that $\delta G$ becomes a cocycle. More precisely, we have the following lemmas.

Lemma 14.3. The previous construction gives rise to a 1-cell in SCG

$$
\delta: \mathcal{B}^{2}(\mathbb{C}, \mathbb{A}) \rightarrow \mathcal{Z}^{2}(\mathbb{C}, \mathbb{A}) .
$$

Lemma 14.4. The kernel of $\delta$ is equivalent, in $\mathrm{SCG}$, to $\operatorname{Hom}(\mathbb{C}, \mathbb{A})$.

Proof. We only explain the construction of the equivalence. First, observe that there is a forgetful functor $U: \operatorname{Hom}(\mathbb{C}, \mathbb{A}) \rightarrow \mathcal{B}^{2}(\mathbb{C}, \mathbb{A})$ which sends $G=\left(G, G_{I}, G_{X, Y}\right)$ into $G=\left(G, G_{I}\right)$. Clearly, $U$ is a 1-cell in SCG. Moreover, $G_{X, Y}$ precisely gives a 2-cell in SCG

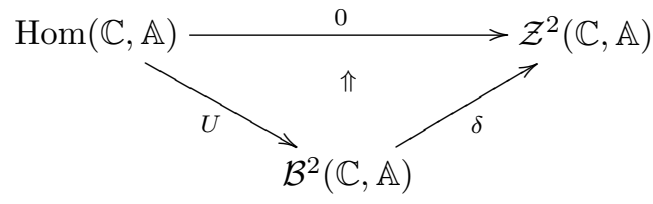

so that $U$ factors through the kernel of $\delta$. This factorization is in fact an equivalence.

To end this section, let us point out some formal consequences of the axioms defining a cocycle. They are useful to complete some proof in the next section.

Lemma 14.5. Let $F=\left(F, c_{F}, r_{F}, l_{F}, s_{F}\right)$ be a cocycle. The following equations hold:

(C5) $l_{F}(I)=r_{F}(I)$;

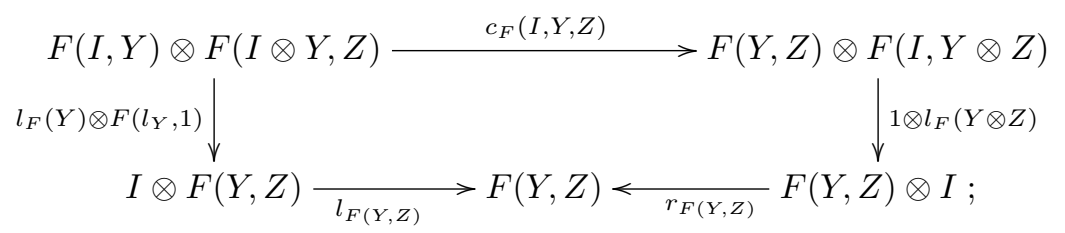

$(C 7)$

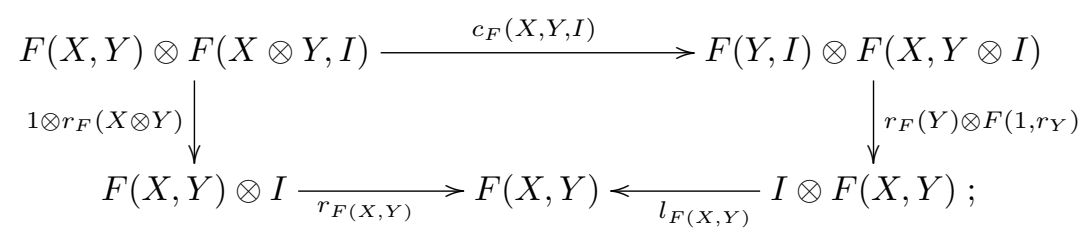


(C8) $s_{F}(X, I) \cdot l_{F}(X)=r_{F}(X), s_{F}(I, Y) \cdot r_{F}(Y)=l_{F}(Y)$;

(C9) $s_{F}(I, I)=1_{F(I, I)}$.

Proof. Similar to that for monoidal categories (see [17] or [15]).

\section{Classification of $\mathcal{F}$-extensions}

¿From the discussion at the beginning of Section 14, we know that each cocycle $F \in \mathcal{Z}^{2}(\mathbb{C}, \mathbb{A})$ induces a symmetric monoidal structure on the groupoid $\mathbb{A} \times \mathbb{C}$, where the tensor product

$$
\otimes_{F}:(\mathbb{A} \times \mathbb{C}) \times(\mathbb{A} \times \mathbb{C}) \rightarrow \mathbb{A} \times \mathbb{C}
$$

is defined by

$$
(A, X) \otimes_{F}(B, Y)=(A \otimes B \otimes F(X, Y), X \otimes Y),
$$

the identity object is $(I, I)$ and the structural isomorphisms are induced by the natural isomorphisms $c_{F}, r_{F}, l_{F}$ and $s_{F}$. We denote by $\mathbb{A} \times_{F} \mathbb{C}$ the category $\mathbb{A} \times \mathbb{C}$ equipped with this structure. It is in fact a symmetric cat-group, a dual being given by $(A, X)^{*}=\left(A^{*} \otimes F\left(X, X^{*}\right)^{*}, X^{*}\right)$.

Proposition 15.1. Let $\mathbb{A}$ and $\mathbb{C}$ be in $\mathrm{SCG}$.

1) The previous construction extends to a functor

$$
\mathcal{E}: \mathcal{Z}^{2}(\mathbb{C}, \mathbb{A}) \rightarrow \mathcal{F}(\mathbb{C}, \mathbb{A}) ;
$$

2) The functor $\mathcal{E}$ is essentially surjective on objects ;

3) The functor $\mathcal{E}$ is a 1-cell in $\mathrm{SCG}$.

Proof. 1) It is a routine verification. Let us only write explicitly the action of $\mathcal{E}$. If $F \in \mathcal{Z}^{2}(\mathbb{C}, \mathbb{A})$, then $\mathcal{E}(F)$ is

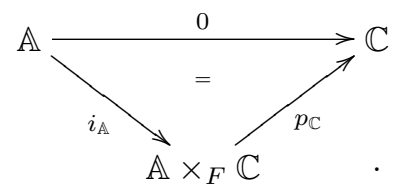

If $\lambda: F \Rightarrow F^{\prime}$ is a morphism in $\mathcal{Z}^{2}(\mathbb{C}, \mathbb{A})$, then $\mathcal{E}(\lambda)$ is

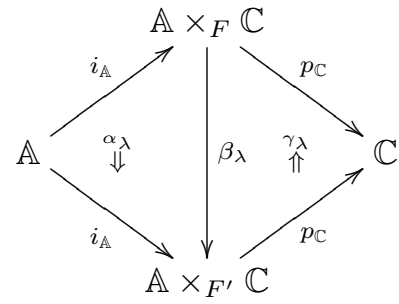


where $\alpha_{\lambda}$ and $\gamma_{\lambda}$ are identity natural transformations and $\beta_{\lambda}$ is the identity as a functor, but its monoidal structure is determined by $\lambda$ in the following way

$$
\begin{aligned}
(A, X) \otimes_{F}(B, Y) & =(A \otimes B \otimes F(X, Y), X \otimes Y) \\
& \downarrow{ }_{\left(1 \otimes 1 \otimes \lambda_{X, Y}, 1 \otimes 1\right)} \\
(A, X) \otimes_{F^{\prime}}(B, Y) & =\left(A \otimes B \otimes F^{\prime}(X, Y), X \otimes Y\right) .
\end{aligned}
$$

2) Let $E=(\Gamma, \varphi, \Sigma): \mathbb{A} \rightarrow \mathbb{B} \rightarrow \mathbb{C}$ be an $\mathcal{F}$-extension with a section

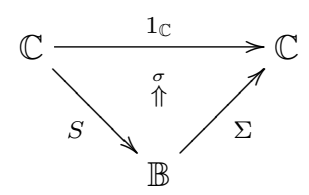

pointed by $S_{I}: I \rightarrow S(I)$. Since $\Gamma_{0}: \mathbb{A} \rightarrow \operatorname{Ker} \Sigma$ is an equivalence, we look for a cocycle $F_{E}: \mathbb{C} \times \mathbb{C} \rightarrow \operatorname{Ker} \Sigma$. If $X$ and $Y$ are in $\mathbb{C}$, we define $F_{E}(X, Y)=(S(X) \otimes$ $\left.S(Y) \otimes S(X \otimes Y)^{*}, \sigma_{X, Y}\right)$, where

$$
\begin{gathered}
\sigma_{X, Y}: \Sigma\left(S(X) \otimes S(Y) \otimes S(X \otimes Y)^{*}\right) \simeq \Sigma(S(X)) \otimes \Sigma(S(Y)) \otimes \Sigma(S(X \otimes Y))^{*} \\
\sigma_{X} \otimes \sigma_{Y} \otimes\left(\sigma_{X \otimes Y}^{-1}\right)^{*} \\
X \otimes Y \otimes(X \otimes Y)^{*} \\
\downarrow^{\mid} \eta_{X \otimes Y}^{-1} \\
I,
\end{gathered}
$$

and $F_{E}(f, g)=S(f) \otimes S(g) \otimes S\left(f^{-1} \otimes g^{-1}\right)^{*}$ for $f$ and $g$ two arrows in $\mathbb{C}$. Now we describe the structure of cocycle of $F_{E}$ :

- $r_{F_{E}}(X): F_{R}(X, I) \rightarrow\left(I, \Sigma_{I}^{-1}: \Sigma(I) \rightarrow I\right)$ is given by

$$
\begin{gathered}
S(X) \otimes S(I) \otimes S(X \otimes I)^{*} \frac{1 \otimes S_{I}^{-1} \otimes S\left(r_{X}^{-1}\right)^{*}}{\downarrow} S(X) \otimes I \otimes S(X)^{*} \\
\qquad \underset{\eta_{S(X)}^{-1}}{\longleftarrow} S(X) \otimes S(X)^{*} ;
\end{gathered}
$$

- $l_{F_{E}}(Y)$ is similar ;

- $s_{F_{E}}(X, Y): F_{E}(X, Y) \rightarrow F_{E}(Y, X)$ is given by

$\gamma_{S(X), S(Y)} \otimes S\left(\gamma_{X, Y}^{-1}\right)^{*}: S(X) \otimes S(Y) \otimes S(X \otimes Y)^{*} \rightarrow S(Y) \otimes S(X) \otimes S(Y \otimes X)^{*} ;$

- $c_{F_{E}}(X, Y, Z): F_{E}(X, Y) \otimes F_{E}(X \otimes Y, Z) \rightarrow F_{E}(Y, Z) \otimes F_{E}(X, Y \otimes Z)$ is given 
by

$$
\begin{aligned}
& S(X) \otimes S(Y) \otimes S(X \otimes Y)^{*} \otimes S(X \otimes Y) \otimes S(Z) \otimes S(X \otimes Y \otimes Z)^{*} \\
& \downarrow 1 \otimes 1 \otimes \epsilon_{S(X \otimes Y)} \otimes 1 \otimes 1 \\
& S(X) \otimes S(Y) \otimes S(Z) \otimes S(X \otimes Y \otimes Z)^{*} \\
& \downarrow 1 \otimes 1 \otimes 1 \otimes \epsilon_{S(Y \otimes Z)}^{-1} \otimes 1 \\
& S(X) \otimes S(Y) \otimes S(Z) \otimes S(Y \otimes Z)^{*} \otimes S(Y \otimes Z) \otimes S(X \otimes Y \otimes Z)^{*} \\
& \gamma_{S(X), S(Y) \otimes S(Z) \otimes S(Y \otimes Z) * \otimes 1 \otimes 1} \\
& S(Y) \otimes S(Z) \otimes S(Y \otimes Z)^{*} \otimes S(X) \otimes S(Y \otimes Z) \otimes S(X \otimes Y \otimes Z)^{*} .
\end{aligned}
$$

It remains to find a 1 -cell in $\mathcal{F}(\mathbb{C}, \operatorname{Ker} \Sigma)$ as in the following diagram

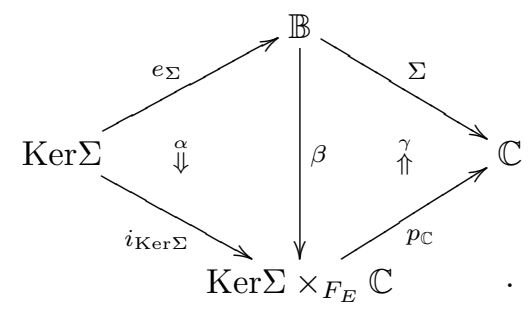

As far as $\beta$ is concerned, if $B$ is in $\mathbb{B}$, put

$$
\beta(B)=\left(B \otimes S(\Sigma(B))^{*},\left(1_{\Sigma(B)} \otimes\left(\sigma_{\Sigma(B)}^{-1}\right)^{*}\right) \cdot \eta_{\Sigma(B)}^{-1}, \Sigma(B)\right)
$$

and $\beta(g)=\left(g \otimes S\left(\Sigma\left(g^{-1}\right)\right)^{*}, \Sigma(g)\right)$ if $g$ is an arrow in $\mathbb{B}$. The monoidal structure of $\beta$ is given by that of $\Sigma$ on the second component, and, up to symmetry, by the counits $\epsilon_{S(\Sigma(A))}$ and $\epsilon_{S(\Sigma(B))}$ on the first component $(A, B$ being two objects in $\mathbb{B})$. Finally, the 2-cell $\gamma$ is the identity natural transformation, and the 2-cell $\alpha$ is given, for an object $(B, b: \Sigma(B) \rightarrow I)$ in $\operatorname{Ker} \Sigma$, by $\left(1 \otimes S\left(\Sigma\left(b^{-1}\right)\right)^{*}, b\right)$.

3) Consider now $F, F^{\prime} \in \mathcal{Z}^{2}(\mathbb{C}, \mathbb{A})$, the direct sum $\mathcal{E}(F) \oplus \mathcal{E}\left(F^{\prime}\right)$ and the $\mathcal{F}$-extension built up using the pullback along the diagonal $\Delta: \mathbb{C} \rightarrow \mathbb{C} \times \mathbb{C}$ as in the following diagram

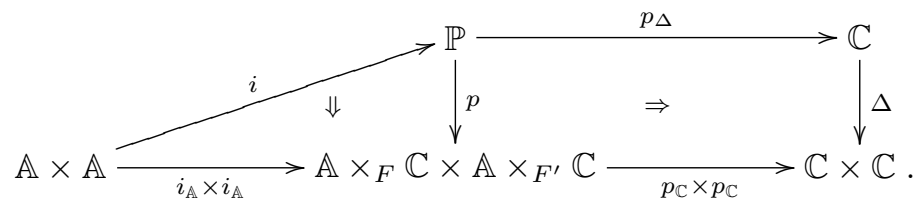

An object in $\mathbb{P}$ is given by $(A, X, B, Y, C, x, y)$, with $A, B$ in $\mathbb{A}, X, Y, C$ in $\mathbb{C}$ and $x: X \rightarrow C, y: Y \rightarrow C$; the arrows are the obvious ones. The functor $i: \mathbb{A} \times \mathbb{A} \rightarrow \mathbb{P}$ sends $(A, B)$ into $(A, I, B, I, I, 1,1)$. Consider also $F \otimes F^{\prime}$ in $\mathcal{Z}^{2}(\mathbb{C}, \mathbb{A})$ and the $\mathcal{F}$ extension

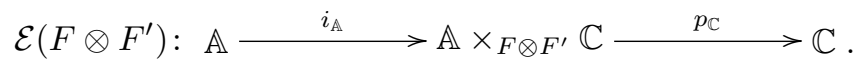


The monoidal structure of $\mathcal{E}$ results from showing that the following diagram is a pushout in SCG :

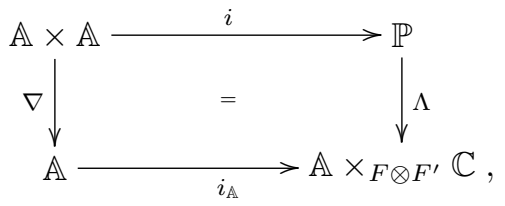

where $\Lambda$ sends $(A, X, B, Y, C, x, y)$ into $(A \otimes B, C)$. If $\left(A^{\prime}, X^{\prime}, B^{\prime}, Y^{\prime}, C^{\prime}, x^{\prime}, y^{\prime}\right)$ is a second object in $\mathbb{P}$, the monoidal structure of $\Lambda$ is given, on the second component, by $F\left(x, x^{\prime}\right)$ and $F^{\prime}\left(y, y^{\prime}\right)$. To show that the previous diagram is a pushout in SCG, consider any diagram of the form

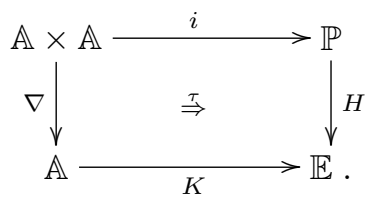

We need a factorization in SCG

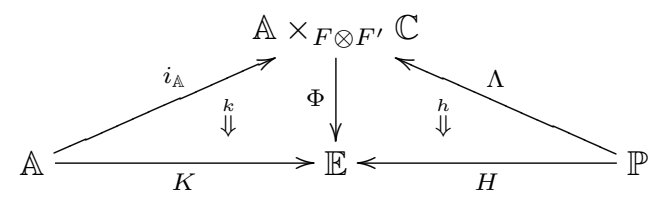

and, for that, we put

- $\Phi(A, X)=K(A) \otimes_{\mathbb{E}} H(I, X, I, X, X, 1,1)$, with the monoidal structure induced by $\tau$;

- $k$ is induced by $H_{I}: I \rightarrow H(I)$ :

- $h$ is defined, using $l_{F}, l_{F^{\prime}}$ and the monoidal structure of $H$, in the following way :

$\Phi(\Lambda(A, X, B, Y, C, x, y))=$

$$
=K(A \otimes B) \otimes_{\mathbb{E}} H(C) \stackrel{\tau_{A, B}^{-1} \otimes 1}{\longrightarrow} H(A, I, B, I, I, 1,1) \otimes_{\mathbb{E}} H(C) \simeq
$$

$\simeq H\left((A, I, B, I, I, 1,1) \otimes_{\mathbb{P}}(I, C, I, C, C, 1,1)\right) \simeq$

$\simeq H\left(A \otimes F(I, C), C, B \otimes F^{\prime}(I, C), C, C, 1,1\right) \simeq$

$$
\simeq H(A, C, B, C, C, 1,1) \stackrel{H\left(1, x^{-1}, 1, y^{-1}, 1\right)}{\longrightarrow} H(A, X, B, Y, C, x, y) .
$$

Finally, given another factorization in SCG

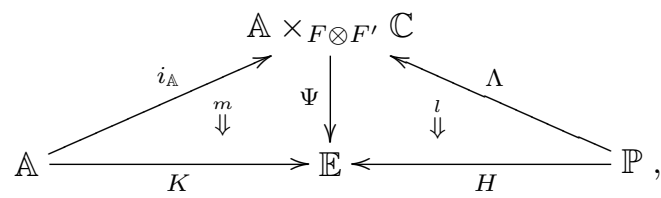


since $i_{\mathbb{A}}(A) \otimes_{F \otimes F^{\prime}} \Lambda(I, C, I, C, C, 1,1)=\left(A \otimes I \otimes F(I, C) \otimes F^{\prime}(I, C), I \otimes C\right) \simeq(A, C)$, we have

$$
\begin{gathered}
\Psi(A, C) \simeq \Psi\left(i_{\mathbb{A}}(A)\right) \otimes_{\mathbb{E}} \Psi(\Lambda(I, C, I, C, C, 1,1)) \\
\qquad m_{A} \otimes l_{(I, C, I, C, C, 1,1)} \\
\downarrow_{\mathbb{E}} H(I, C, I, C, C, 1,1) .
\end{gathered}
$$

To get a cohomological classification of $\mathcal{F}$-extensions, we need a 2 -cell in SCG

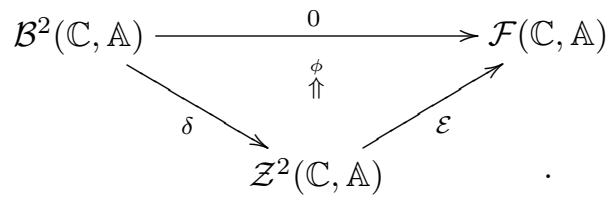

For this, let us fix a cobord $G \in \mathcal{B}^{2}(\mathbb{C}, \mathbb{A})$ and let us describe the following 1-cell in $\mathcal{F}(\mathbb{C}, \mathbb{A})$

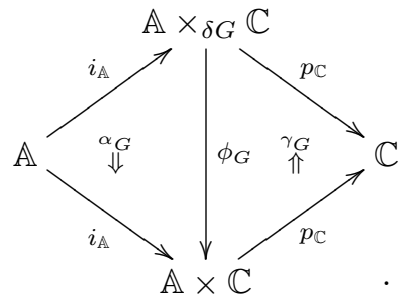

We put $\phi_{G}(A, X)=\left(A \otimes G(X)^{*}, X\right)$ for $(A, X)$ an object of $\mathbb{A} \times_{\delta G} \mathbb{C}$, and $\phi_{G}(f, g)=$ $\left(f \otimes G\left(g^{-1}\right)^{*}, g\right)$ for $(f, g)$ an arrow in $\mathbb{A} \times_{\delta G} \mathbb{C}$. Its monoidal structure is induced by the unit $\eta_{G(X \otimes Y)}$. As far as the 2-cells are concerned, $\gamma_{G}$ is the identity natural transformation, and $\alpha_{G}$ is induced by $G_{I}: I \rightarrow G(I)$. If $\lambda: G \Rightarrow G^{\prime}$ is a morphism in $\mathcal{B}^{2}(\mathbb{C}, \mathbb{A})$, the naturality of $\phi$ is attested by the following 2-cell in $\mathcal{F}(\mathbb{C}, \mathbb{A})$ :

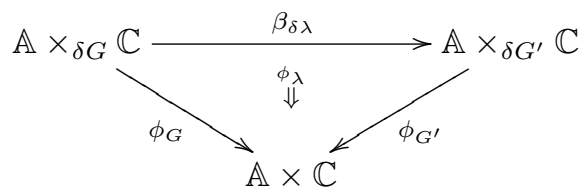

where, for $(A, X)$ an object in $\mathbb{A} \times_{\delta G} \mathbb{C}$, we have :

$$
\phi_{\lambda}(A, X)=\left(1 \otimes \lambda_{X}^{*}, 1\right)^{-1}:\left(A \otimes G^{\prime}(X)^{*}, X\right) \rightarrow\left(A \otimes G(X)^{*}, X\right) .
$$

Consider now the cokernel of $\delta$ :

$$
\mathcal{B}^{2}(\mathbb{C}, \mathbb{A}) \stackrel{\delta}{\longrightarrow} \mathcal{Z}^{2}(\mathbb{C}, \mathbb{A}) \stackrel{P}{\longrightarrow} \operatorname{Coker} \delta
$$

Using its universal property and the 2-cell $\phi: \delta \cdot \mathcal{E} \Rightarrow 0$, we get a 1-cell

$$
\mathcal{E}^{\prime}: \operatorname{Coker} \delta \rightarrow \mathcal{F}(\mathbb{C}, \mathbb{A})
$$


which extends $\mathcal{E}: \mathcal{Z}^{2}(\mathbb{C}, \mathbb{A}) \rightarrow \mathcal{F}(\mathbb{C}, \mathbb{A})$.

Theorem 15.2. The 1-cell in SCG

$$
\mathcal{E}^{\prime}: \operatorname{Coker} \delta \rightarrow \mathcal{F}(\mathbb{C}, \mathbb{A})
$$

is an equivalence.

Proof. Because of point 2 of Proposition 15.1, it is enough to prove that $\mathcal{E}^{\prime}$ is full and faithful. By Proposition 6.2 in [16], this is equivalent to prove that the factorization

$$
\delta^{\prime}: \mathcal{B}^{2}(\mathbb{C}, \mathbb{A}) \rightarrow \operatorname{Ker} \mathcal{E}
$$

of $\delta$ through the kernel of $\mathcal{E}$ (factorization induced by the 2-cell $\phi: \delta \cdot \mathcal{E} \Rightarrow 0$ ) is full and essentially surjective on objects.

Essentially surjective : Consider an object in $\operatorname{Ker} \mathcal{E}$, that is $F \in \mathcal{Z}^{2}(\mathbb{C}, \mathbb{A})$ together with a section in SCG

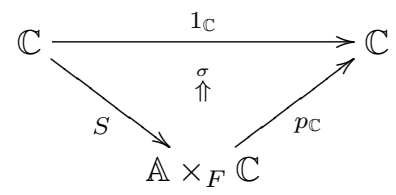

We get a pointed functor

$$
G=S \cdot p_{A}: \mathbb{C} \longrightarrow \mathbb{A} \times_{F} \mathbb{C} \longrightarrow \mathbb{A}
$$

and we need an isomorphism $F \simeq \delta G$. For this, observe that $S(X) \simeq(G(X), X)$ (because of $\sigma$ ), so that the monoidal structure of $S$ gives the following chain of natural isomorphisms : $(G(X \otimes Y), X \otimes Y) \simeq S(X \otimes Y) \simeq S(X) \otimes_{F} S(Y) \simeq$ $(G(X), X) \otimes_{F}(G(Y), Y)=(G(X) \otimes G(Y) \otimes F(X, Y), X \otimes Y)$. Its first component gives a natural isomorphism $F(X, Y) \simeq G(X \otimes Y) \otimes G(Y)^{*} \otimes G(X)^{*}$, as requested. It remains to prove that this isomorphism is an arrow in $\operatorname{Ker} \mathcal{E}$. But this is attested by the following chain of natural isomorphisms, where $\beta: \mathbb{A} \times \mathbb{C} \rightarrow \mathbb{A} \times{ }_{F} \mathbb{C}$ is induced by $i_{\mathbb{A}}: \mathbb{A} \rightarrow \mathbb{A} \times_{F} \mathbb{C}$ and $S: \mathbb{C} \rightarrow \mathbb{A} \times_{F} \mathbb{C}: \phi_{G}(\beta(A, X)) \simeq \phi_{G}\left((A, I) \otimes_{F}(G(X), X)\right)=$ $\phi_{G}(A \otimes G(X) \otimes F(I, X), I \otimes X) \simeq \phi_{G}(A \otimes G(X), X)=\left(A \otimes G(X) \otimes G(X)^{*}, X\right) \simeq$ $(A, X)$.

Full : Consider now $G_{1}, G_{2} \in \mathcal{B}^{2}(\mathbb{C}, \mathbb{A})$ and let $\lambda: \delta G_{1} \Rightarrow \delta G_{2}$ be in $\mathcal{Z}^{2}(\mathbb{C}, \mathbb{A})$. Assume also that there is a 2-cell $s: \phi_{G_{1}} \Rightarrow \beta_{\lambda} \cdot \phi_{G_{2}}$ in $\operatorname{EXT}(\mathbb{C}, \mathbb{A})$. In particular, for each $X$ in $\mathbb{C}$, there is a natural isomorphism $s_{(I, X)}:\left(G_{1}(X)^{*}, X\right) \rightarrow\left(G_{2}(X)^{*}, X\right)$. Its first component induces a natural isomorphism $\bar{s}_{X}: G_{1}(X) \rightarrow G_{2}(X)$ and, using that $s$ is monoidal, one can check that $\delta(\bar{s})=\lambda$.

\section{References}

[1] D. BouRn: The tower of n-groupoids and the long cohomology sequence, J. Pure Applied Algebra 62 (1989) 137-183.

[2] L. Breen: Théorie de Schreier supérieure, Ann. scient. Ec. Norm. Sup. 25 (1992) 465-514. 
[3] M. Bullejos, P. Carrasco, A.M. Cegarra: Cohomology with coefficients in symmetric cat-groups. An extension of Eilenberg-MacLane's classification theorem, Math. Proc. Camb. Phil. Soc. 114 (1993) 163189.

[4] P. Carrasco, A.M. Cegarra: Group-theoretic algebraic models for homotopy types, J. Pure Applied Algebra 75 (1991) 195-235.

[5] P. Carrasco, A.M. Cegarra: (Braided) tensor structures on homotopy groupoids and nerves of (braided) categorical groups, Communications in Algebra 24 (1996) 3995-4058.

[6] P. Carrasco, A.M. Cegarra: Schreier theory for central extensions of categorical groups, Communications in Algebra 24 (1996) 4059-4112.

[7] P. Carrasco, J.M. Moreno: Categorical G-crossed modules and 2fold extensions, J. Pure Applied Algebra 163 (2001) 235-257.

[8] A.M. Cegarra, L. Fernandez: Cohomology of cofibred categorical groups, J. Pure Applied Algebra 143 (1999) 107-154.

[9] A.M. Cegarra, J.M. Garcia-Calcines, J.A. Ortega: Cohomology of groups with operators, Homology, Homotopy and Applications 4 (2002) 1-23 (http://www.rmi.acnet.ge/hha/).

[10] A.M. Cegarra, J.M. Garcia-Calcines, J.A. Ortega: On graded categorical groups and equivariant group extensions, Canad. J. Math. 54 (2002) 970-997.

[11] D. Conduché: Modules croisés généralisés de longueur 2, J. Pure Applied Algebra 34 (1984) 155-178.

[12] P. Deligne: La formule de dualité globale, Springer LNM 305 (1973) 481-587.

[13] A. Frohlich, C.T.C. Wall: Graded monoidal categories, Compositio Mathematica 28 (1974) 229-285.

[14] A.R. Garzon, H. InASSARIDZE: Semidirect products of categorical groups. Obstruction theory, Homology, Homotopy and Applications 3 (2001) 111-138 (http://www.rmi.acnet.ge/hha/).

[15] A. Joyal, R. Street: Braided tensor categories, Advances in Mathematics 102 (1993) 20-78.

[16] S. Kasangian, E.M. Vitale: Factorization systems for symmetric cat-groups, Theory and Applications of Categories 7 (2000) 47-70 (http://www.tac.mta.ca/tac/).

[17] Ch. Kassel: Quantum groups, Springer-Verlag (1995).

[18] G.M. Kelly, M.L. Laplaza: Coherence for compact closed categories, J. Pure Applied Algebra 19 (1980) 193-213.

[19] M.L. LAPLAZA: Coherence for categories with group structure: an alternative approach, J. Algebra 84 (1983) 305-323.

[20] A. Rousseau: Extensions de GR-catégories, Thèse de Doctorat, Université Paris XIII (2000). 
[21] H.X. Sinh: Gr-catégories, Thèse de Doctorat d'Etat, Université Paris VII (1975).

[22] R. Street: Fibrations in bicategories, Cahiers Topologie Géométrie Différentielle 21 (1980) 111-160.

[23] M. TAKeuchi, K.-H. UlbRich: Complexes of categories with abelian group structure, J. Pure Applied Algebra 27 (1983) 61-73.

[24] K.-H. UlbRICH: Kohrenz in Kategorien mit Gruppenstruktur I, II, III, J. Algebra 72 (1981) 279-295, J. Algebra 81 (1983) 278-294, J. Algebra 88 (1984) 292-316.

[25] K.-H. UlbRIch: Group cohomology for Picard categories, J. Algebra 91 (1984) 464-498.

[26] O.E. VillamayoR, D. Zelinsky: Brauer groups and Amitsur cohomology for general commutative ring extensions, J. Pure Applied Algebra 10 (1977) 19-55.

[27] E.M. Vitale: A Picard-Brauer exact sequence of categorical groups, J. Pure Applied Algebra 175 (2002) 383-408.

This article may be accessed via WWW at http://www.rmi.acnet.ge/hha/ or by anonymous ftp at

ftp://ftp.rmi.acnet.ge/pub/hha/volumes/2002/n1a8/v4n1a8.(dvi,ps,pdf)

D. Bourn bourn@lmpa.univ-littoral.fr

Laboratoire de Mathématiques,

Université du Littoral

220 av. de l'Université,

BP 5526, 59379 Dunkerque,

France.

E.M. Vitale vitale@math.ucl.ac.be

Département de Mathématiques,

Université catholique de Louvain

Chemin du Cyclotron 2,

1348 Louvain-la-Neuve,

Belgium. 